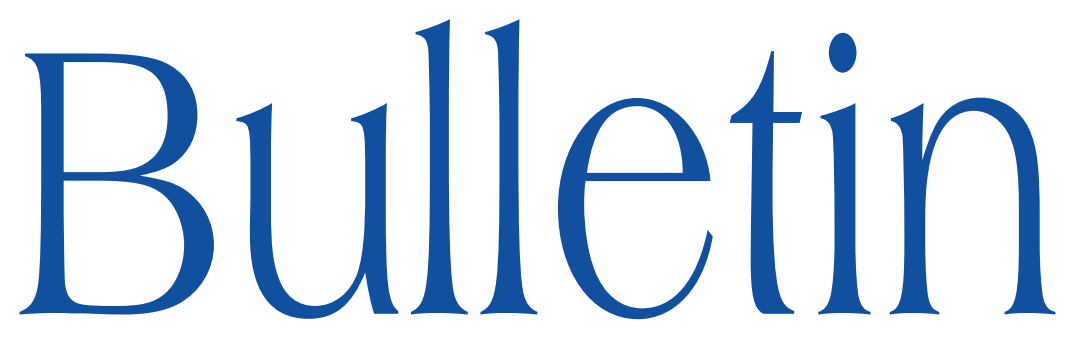

de la SOCIÉTÉ MATHÉMATIQUE DE FRANCE

\title{
CATÉGORIE HOMOTOPIQUE STABLE D'UN SITE SUSPENDU AVEC INTERVALLE
}

Joël Riou

Tome 135

Fascicule 4

2007 


\title{
CATÉGORIE HOMOTOPIQUE STABLE D'UN SITE SUSPENDU AVEC INTERVALLE
}

\author{
PAR JOËL RIOU
}

\begin{abstract}
RÉsumé. - Cet article présente la construction de la catégorie homotopique stable d'un site suspendu avec intervalle arbitraire. La fonctorialité de cette construction est étudiée, avec des applications à la théorie homotopique des schémas introduite par F. Morel et V. Voevodsky.
\end{abstract}

Abstract (The stable homotopy category of an hanging site with interval)

This article describes the construction of the stable homotopy category of an arbitrary hanging site with interval. The functoriality of this construction is studied and has applications to the $\mathbf{A}^{1}$-homotopy theory introduced by F. Morel and V. Voevodsky.

Dans leur article [24], Fabien Morel et Vladimir Voevodsky ont construit la catégorie homotopique $\mathscr{H}(S)$ d'un schéma noethérien $S$. Ce travail s'appuyait sur la construction d'une structure de catégorie de modèles fermée sur la catégorie des faisceaux simpliciaux sur un site (cf. [19], voir aussi [18] et [6] pour des travaux précurseurs).

Certains des énoncés fondamentaux de la théorie homotopique des schémas s'énoncent le plus élégamment dans la catégorie homotopique stable $\mathcal{S H}(S)$

Texte recu le 29 janvier 2007, accepté le 5 septembre 2007

JoËL Rıou, Université Paris-Sud 11, Département de mathématiques, bât. 425, 91405 Orsay Url : http://www.math.u-psud.fr/ riou/

- E-mail : joel.riou@math.u-psud.fr

Classification mathématique par sujets (2000). - 14F20, 14F42, 18F10, 18G30, 18G55, $55 \mathrm{P} 42$.

Mots clefs. - Théorie homotopique des schémas, faisceaux simpliciaux, spectres, catégorie triangulée. 
associée au schéma $S$ (voir [30, propositions 4.11, 4.12 et 4.13]) plutôt que dans la catégorie homotopique instable $\mathscr{H}(S)$. Cette catégorie homotopique stable a été étudiée par Rick Jardine dans [20]; en particulier, une structure monoïdale symétrique y a été construite grâce à l'utilisation des spectres symétriques. Un autre point de vue est développé dans [17].

Cependant, la construction de [20] utilise des propriétés très particulières de la topologie de Nisnevich : un analogue du théorème de Brown-Gersten de [7] pour la topologie de Nisnevich (cf. [24, proposition 1.16, page 100]) et les énoncés de compacité homotopique qu'on peut en déduire (cf. [20, § 2.2]). Ces propriétés de compacité homotopique ne sont pas satisfaites sur les sites arbitraires; on ne peut donc pas appliquer exactement la même méthode pour définir par exemple les variantes étales des catégories $\mathcal{S H}(S)$.

Dans cet article, on se propose de donner une construction de la catégorie homotopique stable d'un «site suspendu avec intervalle $(\mathscr{S}, I, T)$ avec essentiellement le même degré de généralité que dans [24]. Un site suspendu avec intervalle consiste en la donnée d'un site $\mathscr{S}$, d'un préfaisceau simplicial $I$ sur ce site et d'un préfaisceau simplicial pointé $T$. Comme dans [24], on procède à une localisation à la Bousfield pour définir la notion de $I$-équivalence faible entre préfaisceaux simpliciaux sur $\mathscr{S}$. Disposant d'une notion de $I$-équivalence projective (terme à terme) au niveau des $T$-spectres dans la catégorie des préfaisceaux simpliciaux pointés sur $\mathscr{S}$, on procèdera à une nouvelle localisation à la Bousfield par rapport aux $\Omega$-spectres pour obtenir la notion de $I$-équivalence stable, ce qui donnera naissance à la catégorie homotopique stable $\mathcal{S} \mathcal{H}^{T}(\mathscr{S}, I)$. L'existence des localisations à la Bousfield sera assurée par les résultats généraux de [15] sur les catégories de modèles cellulaires (on donne dans l'appendice $\mathrm{A}$ des conditions très simples impliquant les axiomes de ces catégories de modèles cellulaires).

La section 1 est consacrée à la construction de la catégorie homotopique stable $\mathcal{S H}^{T}(\mathscr{S}, I)$. La fonctorialité élémentaire de cette construction est étudiée dans la section 2 : la notion d'application continue raisonnable de sites suspendus avec intervalle y est dégagée, ce qui permet de procéder comme dans [24]. Dans la section 3, on montre que si l'on suppose que $T$ est une suspension, alors la catégorie homotopique stable $\mathcal{S H}^{T}(\mathscr{S}, I)$ est naturellement munie d'une structure de catégorie triangulée; les $T$-espaces de lacets y sont également étudiés, ce qui permet de donner une condition suffisante pour pouvoir définir les équivalences stables à la manière de [20]. La section 4 étudie le cas particulier des catégories homotopiques stables $\mathcal{S H}(S)$ des schémas noethériens; leur fonctorialité élémentaire y est étudiée de façon à permettre la vérification des axiomes des foncteurs homotopiques stables; d'après la thèse de Joseph Ayoub [4], il existe donc une théorie des foncteurs $f^{!}$et $f$ ! pour les catégories $\mathcal{S H}(S)$. La section 5 vise à définir rigoureusement le foncteur «points

TOME $135-2007-\mathrm{N}^{\circ} 4$ 
complexes » $\mathcal{S H}(\mathbf{C}) \rightarrow \mathcal{S H}^{\text {top }}$ : cette construction utilise les résultats de la section 2 et ceux de Daniel Dugger, Sharon Hollander et Daniel Isaksen sur les hyper-recouvrements (cf. [10] et [11]). Enfin, dans la section 6, on propose une définition d'une variante «naïve » de la catégorie $\mathcal{S H}^{T}(\mathscr{S}, I)$ : admettant une description très simple à partie de la catégorie homotopique pointée $\mathscr{H}_{\bullet}(\mathscr{S}, I)$, cette catégorie s'avère être équivalente au quotient de $\mathcal{S H}^{T}(\mathscr{S}, I)$ par l'idéal de morphismes (de carré nul) constitué par les morphismes dits «stablement fantômes ».

Je remercie l'institut de recherche Harish-Chandra à Allahabad (Inde) pour son hospitalité, et son excellent cadre de travail qui m'a permis de finaliser cet article qui est dérivé de ma thèse [26].

\section{Construction}

1.1. Rappels sur la construction de Morel et Voevodsky. - Soit $\mathscr{S}$ un site (c'està-dire une petite catégorie ${ }^{(1)}$, aussi notée $\mathscr{S}$, munie d'une topologie de Grothendieck, cf. SGA 4 II). On note $\operatorname{Prefais}(\mathscr{S})$ la catégorie des préfaisceaux d'ensembles sur la catégorie sous-jacente au site $\mathscr{S}$ et Fais $(\mathscr{S})$ la sous-catégorie pleine de Prefais $(\mathscr{S})$ formée des faisceaux pour la topologie donnée. On note $a: \operatorname{Prefais}(\mathscr{S}) \rightarrow \operatorname{Fais}(\mathscr{S})$ le foncteur faisceau associé, adjoint à gauche de l'inclusion Fais $(\mathscr{S}) \rightarrow \operatorname{Prefais}(\mathscr{S})$.

Définition 1.1. - La catégorie $\operatorname{Esp}(\mathscr{S})$ des espaces sur $\mathscr{S}$ désignera au choix soit la catégorie $\boldsymbol{\Delta}^{\mathrm{opp}} \operatorname{Prefais}(\mathscr{S})$ des préfaisceaux simpliciaux, soit la catégorie $\boldsymbol{\Delta}^{\mathrm{opp}} \mathbf{F a i s}(\mathscr{S})$ des faisceaux simpliciaux.

La notion d'équivalence faible simpliciale dans la catégorie $\operatorname{Esp}(\mathscr{S})$ est définie dans [19]. Une définition équivalente faisant intervenir les faisceaux d'homotopie est suggérée dans [24, remark 1.3, page 48]. Pour la commodité du lecteur, voici une définition possible de la notion d'équivalence faible simpliciale dans le cas particulier des sites ayant suffisamment de points (cf. SGA 4 IV 6.4.1) :

DÉFInItion 1.2. - Soit $\left(\Phi_{i}\right)_{i \in I}$ une famille conservative de foncteurs fibres sur $\mathscr{S}$ indexée par un ensemble $I$. On dit d'un morphisme $f: X \rightarrow Y$ dans $\operatorname{Esp}(\mathscr{S})$ que c'est une équivalence faible simpliciale si pour tout $i \in I$, le morphisme $\Phi_{i}(f): \Phi_{i}(X) \rightarrow \Phi_{i}(Y)$ est une équivalence faible d'ensembles simpliciaux.

(1) Dans les exemples, la catégorie sous-jacente ne sera pas forcément petite, mais seulement essentiellement petite, à savoir équivalente à une petite catégorie. Il sera sous-entendu que l'on remplace la catégorie en question par une petite catégorie qui lui est équivalente; il est facile de vérifier que les catégories construites par la suite pour deux choix initiaux donneront naturellement des catégories équivalentes. 
ThÉORÈme 1.3 (Joyal, Jardine [19]). - Munie des monomorphismes comme cofibrations, des équivalences faibles simpliciales comme équivalences faibles et des morphismes possédant la propriété de relèvement à droite par rapport aux cofibrations triviales comme fibrations, la catégorie $\operatorname{Esp}(\mathscr{S})$ est une catégorie de modèles fermée propre et simpliciale (cf. [25] ou [13]). On note $\mathscr{H}_{\mathrm{s}}(\mathscr{S})$ sa catégorie homotopique (obtenue en inversant formellement les équivalences faibles simpliciales dans $\mathbf{E s p}(\mathscr{S}))$.

À partir de maintenant, on suppose que le site $\mathscr{S}$ est muni d'un objet $I$. La version $I$-localisée de $\mathscr{H}_{\mathrm{s}}(\mathscr{S})$ est obtenue grâce à une localisation à la Bousfield :

DÉFInITION 1.4. - Un objet $X$ de $\mathscr{H}_{\mathrm{s}}(\mathscr{S})$ est $I$-local si pour tout objet $Y$ de $\mathscr{H}_{\mathrm{s}}(\mathscr{S})$, l'application évidente

$$
\operatorname{Hom}_{\mathscr{H}_{\mathrm{s}}(\mathscr{S})}(Y, X) \rightarrow \operatorname{Hom}_{\mathscr{H}_{\mathrm{s}}(\mathscr{S})}(Y \times I, X)
$$

est bijective. Un morphisme $f: X \rightarrow Y$ dans $\operatorname{Esp}(\mathscr{S})$ (ou dans $\mathscr{H}_{\mathrm{s}}(\mathscr{S})$ ) est une $I$-équivalence faible si pour tout objet $I$-local $Z$ de $\mathscr{H}_{\mathrm{s}}(\mathscr{S}), f$ induit une bijection :

$$
\operatorname{Hom}_{\mathscr{H}_{\mathrm{s}}(\mathscr{S})}(Y, Z) \rightarrow \operatorname{Hom}_{\mathscr{H}_{\mathrm{s}}(\mathscr{S})}(X, Z) .
$$

Une $I$-fibration est un morphisme dans $\operatorname{Esp}(\mathscr{S})$ possédant la propriété de relèvement à droite par rapport aux monomorphismes qui sont aussi des $I$ équivalences faibles.

ThÉORÈme 1.5 ([24, theorem 3.2, page 86]). - La catégorie $\mathbf{E s p}(\mathscr{S})$ munie des monomorphismes comme cofibrations, des I-équivalences faibles comme équivalences faibles et des I-fibrations comme fibrations est une catégorie de modèles fermée dont on note $\mathscr{H}(\mathscr{S}, I)$ la catégorie homotopique.

La démonstration de ce théorème dans [24] fait intervenir le résultat technique [24, corollary 2.20, page 77] qui n'y est qu'esquissé. Donnons-en une autre démonstration. Il s'agit de montrer l'existence d'une localisation à la Bousfield. Pour cela, on peut utiliser les techniques générales de [15]. La démonstration du théorème 1.3 montre que la structure simpliciale de catégorie de modèles $\operatorname{sur} \operatorname{Esp}(\mathscr{S})$ est à engendrement cofibrant. Il résulte de la proposition A.4 (voir aussi la remarque A.6) que la structure simpliciale $\operatorname{sur} \operatorname{Esp}(\mathscr{S})$ est cellulaire au sens de [15, definition 12.1.1]. D'après le théorème [15, theorem 4.1.1], pour obtenir l'existence de cette localisation à la Bousfield, il suffit de construire un ensemble de flèches $S$ dans $\operatorname{Esp}(\mathscr{S})$ tel que pour tout objet simplicialement fibrant $X$ de $\operatorname{Esp}(\mathscr{S})$, les deux conditions suivantes soient équivalentes :

- $X$ est $I$-local;

- pour toute flèche $f: A \rightarrow B$ appartenant à $S$, le morphisme d'ensembles simpliciaux $\operatorname{hom}(B, X) \rightarrow \operatorname{hom}(A, X)$ est une équivalence faible.

TOME $135-2007-\mathrm{N}^{\mathrm{O}} 4$ 
Il suffit de prendre pour $S$ l'ensemble des flèches $\operatorname{pr}_{1}: U \times I \rightarrow U$ dans $\operatorname{Esp}(\mathscr{S})$ où $U$ parcourt les objets de la catégorie sous-jacente au site $\mathscr{S}$.

Remarque 1.6. - On appellera abusivement la catégorie homotopique $\mathscr{H}(\mathscr{S}, I)$ la catégorie homotopique du site avec intervalle $(\mathscr{S}, I)$. En effet, il n'a pas été supposé que $I$ était un intervalle au sens de la définition $[24, \S 2.3$, page 85 ] puisque cette hypothèse n'est fondamentalement utilisée que pour permettre la construction de l'objet cosimplicial $\Delta_{I}^{\bullet}$ conduisant notamment à [24, proposition 3.14, page 91] que l'on n'utilisera pas ici. Notons que dans les constructions à venir, ne pas faire cette hypothèse permet d'inclure les versions «non $I$-localisées » des résultats puisqu'ils correspondent au cas particulier où $I$ est l'objet final $\bullet$ de $\operatorname{Esp}(\mathscr{S})$ (au-dessus d'un topos non vide, $\bullet$ n'est jamais un intervalle). Quand «I $»$ sera absent de la notation, il sera sous-entendu que c'est de cette version simpliciale des constructions qu'il s'agit.

Remarque 1.7. - On peut généraliser la définition 1.4 de la façon suivante. Si $A$ est un ensemble des morphismes dans $\mathscr{H}_{\mathrm{s}}(\mathscr{S})$, on peut définir la notion d'objet $A$-local comme dans [24, definition 2.1, page 70] et définir de même les $A$-fibrations et les $A$-équivalences faibles de façon à obtenir mutatis mutandis une version analogue du théorème 1.5 (qui est le cas particulier où la famille $A$ est réduite à un unique morphisme de la forme $I \rightarrow \bullet$ ). Les résultats qui vont suivre seront vrais dans cette généralité : il suffit de remplacer « $I » \operatorname{par}$ « $A »$. L'auteur a préféré conserver « $I$ » puisque c'est le cadre qui l'intéresse le plus, cf. sections 4 et 5 .

Remarque 1.8. - Si on remplace $\operatorname{Esp}(\mathscr{S})$ par la catégorie Esp. $(\mathscr{S})$ des objets pointés dans $\operatorname{Esp}(\mathscr{S})$, on obtient de même une structure de catégorie de modèles $I$-localisée dont on note $\mathscr{H}_{\bullet}(\mathscr{S}, I)$ la catégorie homotopique. Le foncteur d'oubli du point-base $\mathbf{E s p} .(\mathscr{S}) \rightarrow \mathbf{E s p}(\mathscr{S})$ admet un adjoint à gauche $X \longmapsto X \sqcup \bullet$; ces foncteurs induisent une adjonction de Quillen et donc des foncteurs adjoints entre les catégories homotopiques correspondantes ( $I$ localisées ou non).

Remarque 1.9. - La fonctorialité des constructions de ce paragraphe est étudiée à la section 2. Comme le montre [24, example 1.19, page 102], les applications (notamment celles envisagées à la section 4) montrent que l'on ne peut pas se contenter d'étudier la fonctorialité de ces constructions par rapport aux topos et aux morphismes de topos. Il faut affaiblir les hypothèses sur les morphismes. C'est pourquoi les constructions sont faites à partir de la donnée d'un site plutôt que d'un topos et que les morphismes de sites seront remplacés par une notion plus faible. Il est par ailleurs très utile de considérer non seulement les faisceaux simplicaux mais aussi les préfaisceaux simpliciaux : par exemple, les préfaisceaux simpliciaux donnés par les modèles classiques de 
la $K$-théorie algébrique (sur un catégorie convenable de schémas) ne sont pas des faisceaux Zariski.

\subsection{Spectres, structure projective}

DÉFInItion 1.10. - Un site suspendu avec intervalle est un triplet $(\mathscr{S}, I, T)$ où $(\mathscr{S}, I)$ est un «site avec intervalle » et $T$ un objet quelconque de $\operatorname{Esp}_{\bullet}(\mathscr{S})$.

On fixe un site suspendu avec intervalle $(\mathscr{S}, I, T)$ jusqu'à la fin de la section 1.

DÉFInition 1.11. - Un T-spectre $\mathbf{E}$ consiste en la donnée d'une suite $\left(\mathbf{E}_{n}\right)_{n \in \mathbf{N}}$ d'objets de $\mathbf{E s p}_{\bullet}(\mathscr{S})$ et de morphismes (dits d'assemblage) $\sigma_{n}: T \wedge \mathbf{E}_{n} \rightarrow \mathbf{E}_{n+1}$ pour tout entier naturel $n$. Un morphisme de $T$-spectres $f: \mathbf{E} \rightarrow \mathbf{F}$ est une suite de morphismes $f_{n}: \mathbf{E}_{n} \rightarrow \mathbf{F}_{n}$ faisant commuter les diagrammes

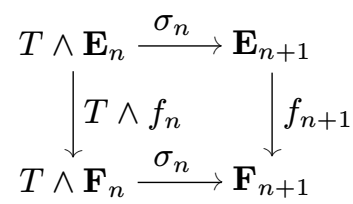

pour tout entier naturel $n$. On note $\mathbf{S p t}^{T}(\mathscr{S})$ la catégorie des $T$-spectres.

Pour tout objet $X$ de $\operatorname{Esp}_{\bullet}(\mathscr{S})$ et tout entier naturel $n$, on note $F_{n} X$ le $T$ spectre défini par $\left(F_{n} X\right)_{k}=\bullet$ pour $k<n$ et $\left(F_{n} X\right)_{n+i}=T^{\wedge i} \wedge X$ pour $i \in \mathbf{N}$, les morphismes d'assemblage $\sigma_{k}$ étant les morphismes triviaux pour $k<n$, le morphisme $\sigma_{n+i}$ étant l'isomorphisme évident $T \wedge\left(F_{n} X\right)_{n+i} \stackrel{\sim}{\rightarrow}\left(F_{n} X\right)_{n+i+1}$ pour $i \in \mathbf{N}$. Pour tout entier naturel $n$, le foncteur $F_{n}: \mathbf{E s p}_{\bullet}(\mathscr{S}) \rightarrow \mathbf{S p t}^{T}(\mathscr{S})$ est le foncteur adjoint à gauche du foncteur $\mathrm{ev}_{n}$ qui à un $T$-spectre $\mathbf{E}$ associe $\mathbf{E}_{n}$. Parfois, le foncteur $F_{0}$ sera implicitement utilisé pour associer naturellement un spectre à un espace pointé.

DÉFInition 1.12. - Une cofibration projective dans $\operatorname{Spt}^{T}(\mathscr{S})$ est un morphisme $f: \mathbf{E} \rightarrow \mathbf{F}$ tel que $\mathbf{E}_{0} \rightarrow \mathbf{F}_{0}$ soit un monomorphisme et que pour tout entier naturel $n$, le morphisme évident

$$
\left(T \wedge \mathbf{F}_{n}\right) \bigvee_{T \wedge \mathbf{E}_{n}} \mathbf{E}_{n+1} \rightarrow \mathbf{F}_{n+1}
$$

soit aussi un monomorphisme. Une $I$-fibration projective (resp. une $I$ équivalence projective) est un morphisme $\mathbf{E} \rightarrow \mathbf{F}$ tel que pour tout entier naturel $n$, le morphisme $\mathbf{E}_{n} \rightarrow \mathbf{F}_{n}$ soit une $I$-fibration (resp. une $I$-équivalence faible) dans $\operatorname{Esp}_{\bullet}(\mathscr{S})$. 
ThÉORÈme 1.13 ([20, lemma 2.1]). - Munie des cofibrations projectives comme cofibrations, des I-équivalences projectives comme équivalences faibles et des I-fibrations projectives comme fibrations, la catégorie $\mathbf{S p t}^{T}(\mathscr{S})$ est une catégorie de modèles fermée propre et simpliciale dont on note $\mathcal{S H}_{\mathrm{p}}^{T}(\mathscr{S}, I)$ la catégorie homotopique.

La structure simpliciale sur $\mathbf{S p t}^{T}(\mathscr{S})$ provient du bifoncteur qui à un ensemble simplicial $K$ et un $T$-spectre $\mathbf{E}$ associe le $T$-spectre $\mathbf{E} \wedge K_{+}$(où le $\wedge$-produit est pris terme à terme).

DÉfinition 1.14. - Une $I$-fibration injective dans $\operatorname{Spt}^{T}(\mathscr{S})$ est un morphisme ayant la propriété de relèvement à droite par rapport aux monomorphismes qui sont aussi des $I$-équivalences projectives.

On dispose d'une autre structure de catégories de modèles fermées dont une bonne vertu est que tous les objets y sont cofibrants :

THÉORÈme 1.15 ([20, lemma 2.1]). - Munie des monomorphismes comme cofibrations, des I-équivalences projectives comme équivalences faibles et des I-fibrations injectives comme fibrations, la catégorie $\mathbf{S p t}^{T}(\mathscr{S})$ est une catégorie de modèles fermée propre et simpliciale.

Pour le moment, les démonstrations données dans [20] dans le cadre des sites avec intervalles $\left(\mathrm{Sm} / S_{\mathrm{Nis}}, \mathbf{A}^{1}\right)$ pour $S$ un schéma de base noethérien s'appliquent encore.

1.3. $\Omega$-spectres, stabilisation. - C'est à partir d'ici que l'on s'écarte de [20], les arguments qui y sont donnés pour construire la catégorie homotopique stable utilisent des propriétés de «compacité » ainsi que la «Nisnevich descent », variante d'un théorème de Brown-Gersten (voir [7], [24, proposition 1.16, page 100] ou encore [28, exercise 2.5] pour le cas des $S^{1}$-spectres) qui est assez spécifique des topologies de Zariski et de Nisnevich sur les schémas noethériens. La construction qui suit s'applique dans un cadre plus général. Nous ferons le lien avec la définition de [20] au théorème 3.35.

Pour tout objet $X$ de $\operatorname{Esp}_{.}(\mathscr{S})$ (cas particulier important : $X=T$ ), le foncteur

$$
X \wedge-: \operatorname{Esp}_{\bullet}(\mathscr{S}) \rightarrow \operatorname{Esp}_{\bullet}(\mathscr{S})
$$

admet un adjoint à droite

$$
\operatorname{Hom}_{\bullet}(X,-): \operatorname{Esp}_{\bullet}(\mathscr{S}) \rightarrow \operatorname{Esp}_{\bullet}(\mathscr{S}),
$$

ce couple de foncteurs adjoints formant une adjonction de Quillen pour la structure $I$-localisée; on note ainsi $\operatorname{R~Hom}_{\bullet}(X,-): \mathscr{H}_{\bullet}(\mathscr{S}, I) \rightarrow \mathscr{H}_{\bullet}(\mathscr{S}, I)$ le foncteur dérivé total à droite de $\operatorname{Hom}_{\bullet}(X,-)$. 
DÉfinition 1.16. - Soit $\mathbf{E}$ un objet $\operatorname{de} \operatorname{Spt}^{T}(\mathscr{S})\left(\right.$ ou $\left.\operatorname{de} \mathcal{S H}_{\mathrm{p}}^{T}(\mathscr{S}, I)\right)$, on dit que $\mathbf{E}$ est un $\Omega$-spectre si pour tout entier naturel $n$, la flèche $\mathbf{E}_{n} \rightarrow$ R Hom. $\left(T, \mathbf{E}_{n+1}\right)$ déduite par adjonction du morphisme d'assemblage $\sigma_{n}: T \wedge$ $\mathbf{E}_{n} \rightarrow \mathbf{E}_{n+1}$ est un isomorphisme dans $\mathscr{H}_{\bullet}(\mathscr{S}, I)$.

DÉFInition 1.17. - On note $\mathcal{S H}_{\Omega}^{T}(\mathscr{S}, I)$ la sous-catégorie pleine de $\mathcal{S H}_{\mathrm{p}}^{T}(\mathscr{S}, I)$ formée des $\Omega$-spectres.

DÉfinition 1.18. - Un morphisme $f: \mathbf{E} \rightarrow \mathbf{F}$ dans $\mathbf{S p t}^{T}(\mathscr{S})$ (ou dans $\left.\mathcal{S} \mathcal{H}_{\mathrm{p}}^{T}(\mathscr{S}, I)\right)$ est une $I$-équivalence stable si pour tout $\Omega$-spectre $\mathbf{G}, f$ induit une bijection

$$
\operatorname{Hom}_{\mathcal{S H}_{\mathrm{p}}^{T}(\mathscr{S}, I)}(\mathbf{F}, \mathbf{G}) \stackrel{\sim}{\rightarrow} \operatorname{Hom}_{\mathcal{S} \mathcal{H}_{\mathrm{p}}^{T}(\mathscr{S}, I)}(\mathbf{E}, \mathbf{G})
$$

DÉFInition 1.19. - Une $I$-fibration stable est un morphisme dans $\operatorname{Spt}^{T}(\mathscr{S})$ ayant la propriété de relèvement à droite par rapport aux cofibrations projectives qui sont aussi des $I$-équivalences stables. Une $I$-fibration injective stable est un morphisme dans $\mathbf{S} \mathbf{S t} \mathbf{t}^{T}(\mathscr{S})$ ayant la propriété de relèvement à droite par rapport aux monomorphismes qui sont aussi des $I$-équivalences stables.

Les deux théorèmes qui suivent donnent des structures de catégories de modèles fermées sur $\mathbf{S p t}^{T}(\mathscr{S})$ ayant pour catégories homotopiques des catégories équivalentes à $\mathcal{S} \mathcal{H}_{\Omega}^{T}(\mathscr{S}, I)$.

THÉORÈme 1.20. - Munie des cofibrations projectives, des I-équivalences stables et des I-fibrations stables, la catégorie $\mathbf{S p t}^{T}(\mathscr{S})$ est une catégorie de modèles fermée simpliciale dont on note $\mathcal{S H}^{T}(\mathscr{S}, I)$ la catégorie homotopique. On appelle $\mathcal{S H}^{T}(\mathscr{S}, I)$ la catégorie homotopique stable du site suspendu avec intervalle $(\mathscr{S}, I, T)$. Le foncteur évident $\mathcal{S H}_{\Omega}^{T}(\mathscr{S}, I) \rightarrow \mathcal{S H}^{T}(\mathscr{S}, I)$ est une équivalence de catégories.

THÉORÈme 1.21. - Munie des monomorphismes comme cofibrations, des Iéquivalences stables et des I-fibrations injectives stables, la catégorie $\mathbf{S p t}^{T}(\mathscr{S})$ est une catégorie de modèles fermée simpliciale (et propre à gauche).

La structure de catégorie de modèles introduite dans le théorème 1.20 sera appelée «structure stable » tandis que celle du théorème 1.21 sera désignée sous le nom de «structure stable injective».

Le lemme suivant montre que les structures de catégories de modèles que l'on veut construire dans ces deux théorèmes sont des localisations à la Bousfield à droite des catégories de modèles des théorèmes 1.13 et 1.15. Elles existent donc en vertu de [15, theorem 4.1.1] dont les hypothèses sont satisfaites (cf. appendice A). 
LEMme 1.22. - Soit $\mathbf{E} \in \mathbf{S p t}^{T}(\mathscr{S})$ un T-spectre projectivement I-fibrant. Alors $\mathbf{E}$ est un $\Omega$-spectre si et seulement si $\mathbf{E}$ est $\mathcal{C}$-local (pour la structure projective) au sens de [15, definition 3.1.4] où $\mathcal{C}$ est la famille des flèches évidentes

$$
F_{n+1}\left(T \wedge X_{+}\right) \rightarrow F_{n} X_{+}
$$

pour $X \in \mathscr{S}$ et $n \in \mathbf{N}$.

Étant donné un $T$-spectre projectivement $I$-fibrant $\mathbf{E}$, dire que $\mathbf{E}$ est un $\Omega$-spectre signifie que pour tout entier naturel $n$, le morphisme canonique

$$
\mathbf{E}_{n} \rightarrow \mathbf{H o m}_{\bullet}\left(T, \mathbf{E}_{n+1}\right)
$$

est une $I$-équivalence faible. Comme ces espaces sont $I$-fibrants, cela signifie encore que pour tout $X \in \mathscr{S}$ et tout $n \in \mathbf{N}$, le morphisme d'ensembles simpliciaux

$$
\mathbf{E}_{n}(X) \rightarrow \mathbf{H o m}_{\bullet}\left(T, \mathbf{E}_{n+1}\right)(X)
$$

est une équivalence faible, autrement dit que le morphisme évident

$$
\operatorname{hom}\left(F_{n} X_{+}, \mathbf{E}\right) \rightarrow \operatorname{hom}\left(F_{n+1}\left(T \wedge X_{+}\right), \mathbf{E}\right)
$$

est une équivalence faible d'ensembles simpliciaux. Comme les objets de la forme $F_{n} X_{+}$et $F_{n+1}\left(T \wedge X_{+}\right)$sont cofibrants pour la structure projective sur $\operatorname{Spt}^{T}(\mathscr{S})$, cela signifie encore que pour tout $f: A \rightarrow B$ dans $\mathcal{C}$, le morphisme induit

$$
\mathrm{R} \operatorname{hom}(B, \mathbf{E}) \rightarrow \mathrm{R} \operatorname{hom}(A, \mathbf{E})
$$

est un isomorphisme dans la catégorie homotopique usuelle $\mathscr{H}^{\text {top }}$, c'est-à-dire que $\mathbf{E}$ est $\mathcal{C}$-local (cf. [15, definition 3.1.4]).

Remarque 1.23. - En fait, pour tout objet $A \in \operatorname{Esp}_{\bullet}(\mathscr{S})$, le morphisme évident $F_{n+1}(T \wedge A) \rightarrow F_{n} A$ est une $I$-équivalence stable. Plus généralement, tout morphisme $\mathbf{E} \rightarrow \mathbf{F}$ dans $\mathbf{S p t}^{T}(\mathscr{S})$ tel que pour tout entier $n$ assez grand le morphisme $\mathbf{E}_{n} \rightarrow \mathbf{F}_{n}$ soit une $I$-équivalence faible est une $I$-équivalence stable (ceci résultera aussitôt du lemme 3.26).

La proposition suivante ne sera pas utilisée dans la suite :

Proposition 1.24. - Le produit de deux I-équivalences stables est une Iéquivalence stable.

Il suffit de montrer que si on fixe un objet $\mathbf{E}$ de $\operatorname{Spt}^{T}(\mathscr{S})$, le foncteur $P: \mathbf{S p t}^{T}(\mathscr{S}) \rightarrow \mathbf{S p t}^{T}(\mathscr{S})$ qui à $\mathbf{F}$ associe $\mathbf{E} \times \mathbf{F}$ préserve les $I$-équivalences stables. Il est tout d'abord trivial que ce foncteur préserve les $I$-équivalences projectives; il reste donc à montrer que pour tout $\mathbf{F} \in \mathbf{S p t}^{T}(\mathscr{S})$, il existe une $I$-équivalence stable (fonctorielle) $\mathbf{F} \rightarrow \mathbf{F}_{\mathrm{s}}$ avec $\mathbf{F}_{\mathrm{s}}$ un $\Omega$-spectre telle que le morphisme $P(\mathbf{F}) \rightarrow P\left(\mathbf{F}_{\mathrm{s}}\right)$ soit une $I$-équivalence stable. 
Soit $\mathcal{J}$ un ensemble de morphismes dans $\mathbf{S p t}^{T}(\mathscr{S})$ engendrant les $I$ cofibrations injectives triviales et $\mathcal{L}$ l'ensemble des morphismes de la forme

$$
\mathbf{A} \wedge \Delta_{+}^{n} \bigsqcup_{\mathbf{A} \wedge \partial_{+}^{n}} \mathbf{B} \wedge \partial \Delta_{+}^{n} \rightarrow \mathbf{B} \wedge \Delta_{+}^{n}
$$

pour $n \in \mathbf{N}$ et $\mathbf{A} \rightarrow \mathbf{B} \in \mathcal{C}$ (cf. lemme 1.22). Un objet de $\mathbf{S p t}^{T}(\mathscr{S})$ est fibrant pour la structure stable injective si et seulement s'il satisfait la propriété de relèvement à droite par rapport aux morphismes de la famille $\mathcal{J} \cup \mathcal{L}$.

On peut donc construire une résolution fibrante pour la structure stable injective en appliquant l'argument du petit objet à cette famille de flèches $\mathcal{J} \cup \mathcal{L}$. Comme le foncteur $P$ préserve les monomorphismes, les sommes amalgamées et les colimites filtrantes, pour conclure que $P(\mathbf{F}) \rightarrow P\left(\mathbf{F}_{\mathrm{s}}\right)$ est une $I$-équivalence stable, il suffit de montrer que pour tout morphisme $f: \mathbf{A} \rightarrow \mathbf{B}$ de $\mathcal{J} \cup \mathcal{L}$, le morphisme $\mathbf{E} \times \mathbf{A} \rightarrow \mathbf{E} \times \mathbf{B}$ est une $I$-équivalence stable. C'est évident si $f \in \mathcal{J}$; pour $f \in \mathcal{L}$, cela résulte de la remarque 1.23. Ceci achève la démonstration de la proposition.

\section{Fonctorialité}

2.1. Changement de site. - On se donne deux sites avec intervalles $\left(\mathscr{S}_{1}, I_{1}\right)$ et $\left(\mathscr{S}_{2}, I_{2}\right)$. On se donne un foncteur $f^{-1}: \mathscr{S}_{2} \rightarrow \mathscr{S}_{1}$ définissant une application continue de sites $f: \mathscr{S}_{1} \rightarrow \mathscr{S}_{2}{ }^{(2)}$. On en déduit formellement un couple de foncteurs adjoints $\left(f^{\star}, f_{\star}\right)$ avec $f_{\star}: \operatorname{Esp}\left(\mathscr{S}_{1}\right) \rightarrow \operatorname{Esp}\left(\mathscr{S}_{2}\right)$ et $f^{\star}: \operatorname{Esp}\left(\mathscr{S}_{2}\right) \rightarrow$ $\operatorname{Esp}\left(\mathscr{S}_{1}\right)$.

DÉFInITION 2.1 ([24, definition 1.55, page 65]). — L'application continue de sites $f: \mathscr{S}_{1} \rightarrow \mathscr{S}_{2}$ est dite raisonnable si pour tout objet $X$ de $\mathscr{S}_{2}$, tout objet simplicialement fibrant $Y$ de $\operatorname{Esp}\left(\mathscr{S}_{1}\right)$ et toute équivalence faible simpliciale $f_{\star} Y \rightarrow Y^{\prime}$ dans $\operatorname{Esp}\left(\mathscr{S}_{2}\right)$ avec $Y^{\prime}$ simplicialement fibrant, l'application évidente d'ensembles simpliciaux

$$
\operatorname{hom}\left(X, f_{\star} Y\right) \rightarrow \operatorname{hom}\left(X, Y^{\prime}\right)
$$

est une équivalence faible.

On suppose que $f$ est une application raisonnable de sites. On se donne de plus un objet $T_{1}$ dans Esp. $\left(\mathscr{S}_{1}\right)$ et un objet $T_{2}$ dans Esp• $\left(\mathscr{S}_{2}\right)$ (autrement dit, on a deux sites suspendus avec intervalles, cf. définition 1.10).

Observons tout d'abord que $f^{-1}$ induit un foncteur $f_{\star}: \operatorname{Esp}_{\bullet}\left(\mathscr{S}_{1}\right) \rightarrow$ Esp. $\left(\mathscr{S}_{2}\right)$ (compatible au foncteur d'oubli du point-base) et que ce foncteur

(2) Autrement dit, le foncteur $f_{\star}: \operatorname{Prefais}\left(\mathscr{S}_{1}\right) \rightarrow \operatorname{Prefais}\left(\mathscr{S}_{2}\right)$ défini à partir de $f^{-1}$ au niveau des catégories de préfaisceaux d'ensembles envoie les faisceaux sur $\mathscr{S}_{1}$ sur des faisceaux $\operatorname{sur} \mathscr{S}_{2}$.

TOME $135-2007-\mathrm{N}^{\mathrm{O}} 4$ 
$f_{\star}: \operatorname{Esp}_{\bullet}\left(\mathscr{S}_{1}\right) \rightarrow \operatorname{Esp}_{\bullet}\left(\mathscr{S}_{2}\right)$ admet un adjoint à gauche $f^{\star}: \mathbf{E s p}_{\bullet}\left(\mathscr{S}_{2}\right) \rightarrow$ Esp. $\left(\mathscr{S}_{1}\right)^{(3)}$.

On se donne une fois pour toutes un isomorphisme de foncteurs $\mathbf{E s p}_{\bullet}\left(\mathscr{S}_{2}\right) \rightarrow$ Esp. $\left(\mathscr{S}_{1}\right)$ en la variable $X$ :

$$
\Psi_{X}: f^{\star}\left(T_{2} \wedge X\right) \stackrel{\sim}{\rightarrow} T_{1} \wedge f^{\star} X
$$

Par adjonction, la donnée de $\Psi$ équivaut à la donnée d'un isomorphisme de foncteurs $\Psi^{\prime}: \mathbf{E s p}_{\bullet}\left(\mathscr{S}_{1}\right) \rightarrow \mathbf{E s p}_{\bullet}\left(\mathscr{S}_{2}\right)$ en la variable $Y$ :

$$
\Psi_{Y}^{\prime}: \operatorname{Hom}_{\bullet}\left(T_{2}, f_{\star} Y\right) \stackrel{\sim}{\rightarrow} f_{\star} \operatorname{Hom}_{\bullet}\left(T_{1}, Y\right) .
$$

On peut alors définir un foncteur $f^{\star}: \mathbf{S p t}^{T_{2}}\left(\mathscr{S}_{2}\right) \rightarrow \mathbf{S p t}^{T_{1}}\left(\mathscr{S}_{1}\right)$ par la formule $\left(f^{\star} \mathbf{E}\right)_{n}=f^{\star}\left(\mathbf{E}_{n}\right)$ pour tout objet $\mathbf{E}$ de $\mathbf{S p t}^{T_{2}}\left(\mathscr{S}_{2}\right)$, les morphismes d'assemblage étant définis en appliquant $f^{\star}$ au morphisme d'assemblage de $\mathbf{E}$ et en utilisant $\Psi$ :

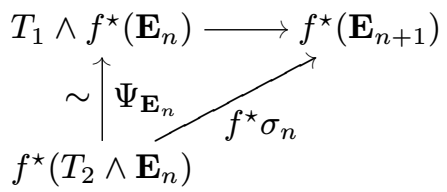

On définit de même un foncteur $f_{\star}: \mathbf{S p t}^{T_{1}}\left(\mathscr{S}_{1}\right) \rightarrow \mathbf{S} \mathbf{p} \mathbf{t}^{T_{2}}\left(\mathscr{S}_{2}\right)$ par la formule $\left(f_{\star} \mathbf{E}\right)_{n}=f_{\star}\left(\mathbf{E}_{n}\right)$ pour tout objet $\mathbf{E}$ de $\mathbf{S} \mathbf{p t} \mathbf{t}^{T_{1}}\left(\mathscr{S}_{1}\right)$, les morphismes adjoints des morphisme d'assemblage de $f_{\star} \mathbf{E}$ étant obtenus en utilisant l'isomorphisme $\Psi^{\prime}$ et en appliquant $f_{\star}$ aux morphismes adjoints $\sigma_{n}^{\prime}$ des morphismes d'assemblage de $\mathbf{E}$ :

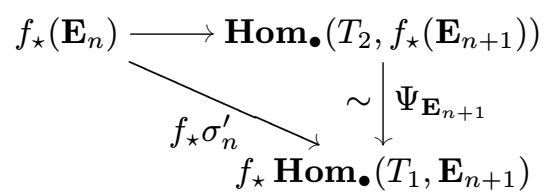

Il est clair que le foncteur $f^{\star}: \mathbf{S p t}^{T_{2}}\left(\mathscr{S}_{2}\right) \rightarrow \mathbf{S p t}^{T_{1}}\left(\mathscr{S}_{1}\right)$ est l'adjoint à gauche du foncteur $f_{\star}: \mathbf{S p t}^{T_{1}}\left(\mathscr{S}_{1}\right) \rightarrow \mathbf{S p t}^{T_{2}}\left(\mathscr{S}_{2}\right)$.

On suppose maintenant que $f:\left(\mathscr{S}_{1}, I_{1}\right) \rightarrow\left(\mathscr{S}_{2}, I_{2}\right)$ constitue une application raisonnable de sites avec intervalles (cf. [24, definition 3.16, page 92]), autrement

(3) Il convient de noter que ce foncteur ne commute pas forcément à l'oubli du point-base (en revanche, dans l'autre sens, il commute à la construction $X \rightarrow X_{+}$consistant à ajouter formellement un point-base à un objet non pointé). Par exemple, si $X$ est un espace topologique, $U$ un ouvert qui n'est pas tout, et que l'on note $j: U \rightarrow X$ l'inclusion canonique, le foncteur d'inclusion $j_{\sharp}$ de la catégorie des ouverts de $U$ dans celle de $X$ induit une application continue de sites $X \rightarrow U$ dont le foncteur image directe est le foncteur $j^{\star}$ usuel et l'adjoint à gauche $j_{\sharp}$ est soit le prolongement par le vide pour les faisceaux d'ensembles, soit le prolongement par • (resp. zéro) pour les faisceaux d'ensembles pointés (resp. de groupes abéliens). 
dit que le foncteur $\mathrm{R} f_{\star}: \mathscr{H}_{\mathrm{S}}\left(\mathscr{S}_{1}\right) \rightarrow \mathscr{H}_{\mathrm{S}}\left(\mathscr{S}_{2}\right)^{(4)}$ envoie les objets $I_{1}$-locaux sur des objets $I_{2}$-locaux.

Lemme 2.2. - Soit $g: \mathbf{E} \rightarrow \mathbf{F}$ une $I_{1}$-équivalence projective entre objets projectivement $I_{1}$-fibrants de $\mathbf{S p t}^{T_{1}}\left(\mathscr{S}_{1}, I_{1}\right)$. Alors $f_{\star} g: f_{\star} \mathbf{E} \rightarrow f_{\star} \mathbf{F}$ est une $I_{2}$ équivalence projective.

C'est évident. On en déduit un foncteur dérivé total à droite

$$
\mathrm{R}_{\mathrm{p}} f_{\star}: \mathcal{S H}_{\mathrm{p}}^{T_{1}}\left(\mathscr{S}_{1}, I_{1}\right) \rightarrow \mathcal{S} \mathcal{H}_{\mathrm{p}}^{T_{2}}\left(\mathscr{S}_{2}, I_{2}\right)
$$

Proposition 2.3. - Le foncteur $\mathrm{R}_{\mathrm{p}} f_{\star}: \mathcal{S H}_{\mathrm{p}}^{T_{1}}\left(\mathscr{S}_{1}, I_{1}\right) \rightarrow \mathcal{S H}_{\mathrm{p}}^{T_{2}}\left(\mathscr{S}_{2}, I_{2}\right)$ admet un adjoint à gauche qui n'est autre que le foncteur dérivé total à gauche de $f^{\star}: \mathbf{S p t}^{T_{2}}\left(\mathscr{S}_{2}\right) \rightarrow \mathbf{S p t}^{T_{1}}\left(\mathscr{S}_{1}\right)$, que l'on note $\mathrm{L} f^{\star}: \mathcal{S H}_{\mathrm{p}}^{T_{2}}\left(\mathscr{S}_{2}, I_{2}\right) \rightarrow$ $\mathcal{S H}_{\mathrm{p}}^{T_{1}}\left(\mathscr{S}_{1}, I_{1}\right)$.

On procède d'une façon tout à fait semblable à celle de [24, proposition 1.57 , page 65] :

Définition 2.4. - Soit $\mathbf{X}$ un objet de $\mathbf{S p t}^{T_{2}}\left(\mathscr{S}_{2}\right)$. On dit que $\mathbf{X}$ est $f^{\star}$ admissible si pour tout objet injectivement $I_{1}$-fibrant $\mathbf{Y} \operatorname{de} \mathbf{S p t}^{T_{1}}\left(\mathscr{S}_{1}\right)$ et toute $I_{2}$-équivalence projective $f_{\star} \mathbf{Y} \rightarrow \mathbf{Y}^{\prime}$ avec $\mathbf{Y}^{\prime}$ un objet injectivement $I_{2}$-fibrant $\operatorname{de}_{\mathbf{S p t}}{ }^{T_{2}}\left(\mathscr{S}_{2}\right)$, la flèche évidente d'ensembles simpliciaux

$$
\operatorname{hom}\left(\mathbf{X}, f_{\star} \mathbf{Y}\right) \rightarrow \operatorname{hom}\left(\mathbf{X}, \mathbf{Y}^{\prime}\right)
$$

est une équivalence faible.

Lemme 2.5. - Soit $\mathbf{X}$ un objet $f^{\star}$-admissible de $\mathbf{S p t}^{T_{2}}\left(\mathscr{S}_{2}\right)$. Alors, le foncteur qui à $\mathbf{Y} \in \operatorname{Spt}^{T_{1}}\left(\mathscr{S}_{1}\right)$ associe l'ensemble $\operatorname{Hom}_{\mathcal{S H}_{\mathrm{p}}\left(\mathscr{S}_{2}, I_{2}\right)}\left(\mathbf{X}, \mathrm{R}_{\mathrm{p}} f_{\star} \mathbf{Y}\right)$ est coreprésentable, c'est-à-dire que "le foncteur adjoint à gauche de $\mathrm{R}_{\mathrm{p}} f_{\star}: \mathcal{S H}_{\mathrm{p}}^{T_{1}}\left(\mathscr{S}_{1}, I_{1}\right) \rightarrow \mathcal{S H}_{\mathrm{p}}^{T_{2}}\left(\mathscr{S}_{2}, I_{2}\right)$ est défini en $\mathbf{X} »$. Plus précisément, on a un isomorphisme fonctoriel en $\mathbf{Y} \in \mathcal{S H}_{\mathrm{p}}^{T_{1}}\left(\mathscr{S}_{1}, I_{1}\right)$ :

$$
\operatorname{Hom}_{\mathcal{S} \mathcal{H}_{\mathrm{p}}^{T_{1}}\left(\mathscr{S}_{1}, I_{1}\right)}\left(f^{\star} \mathbf{X}, \mathbf{Y}\right) \stackrel{\sim}{\rightarrow} \operatorname{Hom}_{\mathcal{S} \mathcal{H}_{\mathrm{p}}^{T_{2}}\left(\mathscr{S}_{2}, I_{2}\right)}\left(\mathbf{X}, \mathrm{R}_{\mathrm{p}} f_{\star} \mathbf{Y}\right) .
$$

En particulier, si $X \rightarrow X^{\prime}$ est une $I_{2}$-équivalence projective entre objets $f^{\star}$ admissibles de $\mathbf{S p t}^{T_{2}}\left(\mathscr{S}_{2}\right)$, alors $f^{\star} X \rightarrow f^{\star} X^{\prime}$ est une $I_{1}$-équivalence projective.

(4) Il s'agit là du foncteur dérivé à droite du foncteur $f_{\star}: \Delta^{\mathrm{opp}}$ Fais $\left(\mathscr{S}_{1}\right) \rightarrow \boldsymbol{\Delta}^{\mathrm{opp}}$ Fais $\left(\mathscr{S}_{2}\right)$ (il est simplement obtenu en appliquant $f_{\star}$ à une résolution fibrante). Cette construction est possible (cf. [24, page 62]) car on a supposé que $f$ était une application continue de sites.

TOME $135-2007-\mathrm{N}^{\mathrm{O}} 4$ 
Pour $k \in\{1,2\}$, on choisit une résolution $I_{k}$-injectivement fibrante $\mathbf{E} \rightarrow \mathbf{E}_{\mathrm{fib}}$ fonctorielle en $Y \in \operatorname{Spt}^{T_{k}}\left(\mathscr{S}_{k}\right)$ (il sera évident que les isomorphismes construits plus bas seront indépendants de ce choix). Pour tout objet $\mathbf{Y} \operatorname{de} \mathbf{S p t}^{T_{1}}\left(\mathscr{S}_{1}\right)$, on considère l'application suivante :

$$
\operatorname{hom}\left(\mathbf{X}, f_{\star}\left(\mathbf{Y}_{\text {fib }}\right)\right) \rightarrow \operatorname{hom}\left(\mathbf{X},\left(f_{\star}\left(\mathbf{Y}_{\text {fib }}\right)\right)_{\text {fib }}\right) .
$$

Comme $\mathbf{X}$ est $f^{\star}$-admissible, cette application est une équivalence faible d'ensembles simpliciaux. On a par ailleurs un isomorphisme canonique d'ensembles simpliciaux :

$$
\operatorname{hom}\left(f^{\star} \mathbf{X}, \mathbf{Y}_{\mathrm{fib}}\right) \stackrel{\sim}{\rightarrow} \operatorname{hom}\left(\mathbf{X}, f_{\star}\left(\mathbf{Y}_{\mathrm{fib}}\right)\right) .
$$

Finalement, on obtient une équivalence faible fonctorielle en $Y$ :

$$
\operatorname{hom}\left(f^{\star} \mathbf{X}, \mathbf{Y}_{\text {fib }}\right) \rightarrow \operatorname{hom}\left(\mathbf{X},\left(f_{\star}\left(\mathbf{Y}_{\text {fib }}\right)\right)_{\text {fib }}\right) .
$$

En passant au $\pi_{0}$, il vient une bijection fonctorielle en $Y$ :

$$
\operatorname{Hom}_{\mathcal{S H}_{\mathrm{p}}^{T_{1}}\left(\mathscr{S}_{1}, I_{1}\right)}\left(f^{\star} \mathbf{X}, \mathbf{Y}\right) \rightarrow \operatorname{Hom}_{\mathcal{S} \mathcal{H}_{\mathrm{p}}^{T_{2}}\left(\mathscr{S}_{2}, I_{2}\right)}\left(\mathbf{X}, \mathrm{R}_{\mathrm{p}} f_{\star} \mathbf{Y}\right)
$$

ce qui achève la démonstration du lemme.

Bref, pour montrer que $\mathrm{R}_{\mathrm{p}} f_{\star}$ admet un adjoint à gauche, il suffit de montrer que tout objet de $\mathcal{S H}_{\mathrm{p}}^{T_{2}}\left(\mathscr{S}_{2}, I_{2}\right)$ peut être représenté par un objet $f^{\star}$-admissible de $\mathbf{S p t}^{T_{2}}\left(\mathscr{S}_{2}\right)$. Le lemme suivant nous donne cela (et permet aussi de montrer que le foncteur adjoint à gauche de $\mathrm{R}_{\mathrm{p}} f_{\star}$ est le foncteur dérivé total à gauche $\left.\operatorname{de} f^{\star}\right)$ :

Lemme 2.6. - Il existe un foncteur $\mathbf{S} \mathbf{S t}^{T_{2}}\left(\mathscr{S}_{2}\right) \rightarrow \mathbf{S p t} \mathbf{t}^{T_{2}}\left(\mathscr{S}_{2}\right)$ qui à un objet $\mathbf{X}$ associe un objet $\mathbf{X}_{\mathrm{adm}} f^{\star}$-admissible, et une transformation naturelle $\mathbf{X}_{\mathrm{adm}} \rightarrow \mathbf{X}$ qui soit une $I_{2}$-équivalence projective.

On applique le raisonnement du petit objet à la famille de flèches $\mathscr{A}$ dans $\operatorname{Spt}^{T_{2}}\left(\mathscr{S}_{2}\right)$ de la forme $F_{n}(A \times X)_{+} \rightarrow F_{n}(B \times X)_{+}$où $X \in \mathscr{S}_{2}, n$ est un entier naturel et $A \rightarrow B$ une inclusion entre ensembles simpliciaux finis. On obtient ainsi une factorisation fonctorielle $\bullet \rightarrow \mathbf{X}_{\mathrm{adm}} \rightarrow \mathbf{X}$ de $\bullet \rightarrow \mathbf{X}$ pour tout objet $\mathbf{X}$ de $\mathbf{S p t}^{T_{2}}\left(\mathscr{S}_{2}\right)$, où $\mathbf{X}_{\text {adm }} \rightarrow \mathbf{X}$ possède la propriété de relèvement à droite par rapport à $\mathscr{A}$ (il s'agit donc d'une $I_{1}$-équivalence projective) et où $\bullet \rightarrow \mathbf{X}_{\text {adm }}$ est un composé transfini d'images directes de sommes directes de morphismes dans $\mathscr{A}$. Observons tout d'abord que les flèches dans $\mathscr{A}$ sont des monomorphismes qui le restent après application de $f^{\star}$; par ailleurs, pour tout entier naturel $n$, tout objet $X \in \mathscr{S}_{2}$ et tout ensemble simplicial $K$, l'objet $F_{n}(K \times X)_{+}$est $f^{\star}$-admissible (cela résulte aussitôt du fait qu'on a une application raisonnable de sites avec intervalles).

Les objets $f^{\star}$-admissibles possèdent des propriétés analogues à celles énoncées dans [24, lemma 1.53, page 64]. Dans le cas où on utilise la catégorie des préfaisceaux pour définir les catégories de spectres, elles permettent de conclure. 
Si on travaille avec des faisceaux, les objets de la forme $F_{n}(A \times X)_{+}$pour $A$ un ensemble simplicial fini et $X \in \mathscr{S}_{2}$ ne sont pas forcément de présentation finie dans $\mathbf{S p t}^{T_{2}}\left(\mathscr{S}_{2}\right)$; pour terminer la démonstration de ce lemme (et donc de la proposition 2.3), on peut utiliser le lemme suivant :

LEMme 2.7. - Soit $\left(X_{i}\right)_{i \in \mathscr{I}}$ un système inductif dans $\mathbf{S p t}^{T_{2}}\left(\mathscr{S}_{2}\right)$ indexé par une catégorie filtrante $\mathscr{I}$. On suppose que pour tout $i \in \mathscr{I}$, l'objet $X_{i}$ est $f^{\star}$-admissible. Alors $\operatorname{colim}_{\mathscr{I}} X_{\bullet}$ est $f^{\star}$-admissible.

Ce lemme résulte facilement du lemme général suivant et du fait que les morphismes évidents hocolim $X_{\bullet} \rightarrow \underset{\mathscr{I}}{\operatorname{colim}} X_{\bullet}$ et $f^{\star} \underset{\mathscr{I}}{\operatorname{hocolim}} X_{\bullet} \rightarrow f^{\star} \operatorname{colim} X_{\mathscr{I}}$ soient des équivalences faibles :

LEMME 2.8. - Soit $\left(X_{i}\right)_{i \in \mathscr{I}}$ un système inductif dans $\mathbf{S p t}^{T_{2}}\left(\mathscr{S}_{2}\right)$ indexé par une catégorie $\mathscr{I}$. On suppose que pour tout $i \in \mathscr{I}$, l'objet $X_{i}$ est $f^{\star}$-admissible. Alors hocolim $X_{\bullet}$ est $f^{\star}$-admissible.

Soit $Y$ un objet injectivement $I_{1}$-fibrant de $\mathbf{S p t}^{T_{1}}\left(\mathscr{S}_{1}\right)$, soit $f_{\star} Y \rightarrow Y^{\prime}$ une résolution injectivement $I_{2}$-fibrante $f_{\star} Y$. Par hypothèse, pour tout $i \in \mathscr{I}$, l'application

$$
\operatorname{hom}\left(X_{i}, f_{\star} Y\right) \rightarrow \operatorname{hom}\left(X_{i}, Y^{\prime}\right)
$$

est une équivalence faible entre ensembles simpliciaux fibrants. On peut passer à la limite homotopique pour obtenir que

$$
\operatorname{hom}\left(\underset{\mathscr{I}}{\operatorname{hocolim}} X_{\bullet}, f_{\star} Y\right) \rightarrow \operatorname{hom}\left(\underset{\mathscr{I}}{\operatorname{hocolim}} X_{\bullet}, Y^{\prime}\right)
$$

est une équivalence faible. On obtient ainsi que hocolim $X_{\mathscr{I}}$ est $f^{\star}$-admissible.

Pour pouvoir étendre convenablement ces constructions aux catégories homotopiques stables, on a besoin d'une hypothèse supplémentaire donnée dans la définition qui vient (et qui récapitule les hypothèses utilisées jusqu'ici) :

DÉfinition 2.9. - On se donne $\left(\mathscr{S}_{1}, I_{1}, T_{1}\right)$ et $\left(\mathscr{S}_{2}, I_{2}, T_{2}\right)$ deux sites suspendus avec intervalles. Une application raisonnable $f:\left(\mathscr{S}_{1}, I_{1}, T_{1}\right) \rightarrow\left(\mathscr{S}_{2}, I_{2}, T_{2}\right)$ de sites suspendus avec intervalles consiste en la donnée d'une application raisonnable $f:\left(\mathscr{S}_{1}, I_{1}\right) \rightarrow\left(\mathscr{S}_{2}, I_{2}\right)$ de sites avec intervalles et d'un isomorphisme de foncteurs

$$
\Psi: f^{\star}\left(T_{2} \wedge-\right) \stackrel{\sim}{\rightarrow} T_{1} \wedge f^{\star}-: \operatorname{Esp}_{\bullet}\left(\mathscr{S}_{2}\right) \rightarrow \operatorname{Esp}_{\bullet}\left(\mathscr{S}_{1}\right)
$$

tels que pour tout objet $I_{1}$-fibrant $X$ de $\operatorname{Esp}_{\bullet}\left(\mathscr{S}_{1}\right)$ et toute $I_{2}$-équivalence faible $f_{\star} X \rightarrow X^{\prime}$ avec $X^{\prime} I_{2}$-fibrant, la flèche Hom. $\left(T_{2}, f_{\star} X\right) \rightarrow$ Hom. $_{\bullet}\left(T_{2}, X^{\prime}\right)$ soit une $I_{2}$-équivalence faible. 
Proposition 2.10. - Soit $f:\left(\mathscr{S}_{1}, I_{1}, T_{1}\right) \rightarrow\left(\mathscr{S}_{2}, I_{2}, T_{2}\right)$ une application raisonnable de sites suspendus avec intervalles. Alors, le foncteur $\mathrm{R}_{\mathrm{p}} f_{\star}: \mathcal{S H}_{\mathrm{p}}^{T_{1}}\left(\mathscr{S}_{1}, I_{1}\right) \rightarrow \mathcal{S H}_{\mathrm{p}}^{T_{2}}\left(\mathscr{S}_{2}, I_{2}\right)$ est tel que

$$
\mathrm{R}_{\mathrm{p}} f_{\star}\left(\mathcal{S} \mathcal{H}_{\Omega}^{T_{1}}\left(\mathscr{S}_{1}, I_{1}\right)\right) \subset \mathcal{S} \mathcal{H}_{\Omega}^{T_{2}}\left(\mathscr{S}_{2}, I_{2}\right) .
$$

Si $g: \mathbf{E} \rightarrow \mathbf{F}$ est un morphisme dans $\mathbf{S p t}^{T_{1}}\left(\mathscr{S}_{1}\right)$ qui est une $I_{1}$-équivalence stable entre $\Omega$-spectres $I_{1}$-projectivement fibrants, alors $f_{\star} g: f_{\star} \mathbf{E} \rightarrow f_{\star} \mathbf{G}$ est une $I_{2}$-équivalence stable (entre $\Omega$-spectres).

Le fait que $\mathrm{R}_{\mathrm{p}} f_{\star}$ préserve les $\Omega$-spectres résulte de la dernière condition intervenant dans la définition d'une application raisonnable de sites suspendus avec intervalles. La deuxième assertion en découle en vertu du lemme 2.2.

Sous les hypothèses de la proposition, on obtient aussitôt un foncteur dérivé total à droite $\mathrm{R} f_{\star}: \mathcal{S} \mathcal{H}^{T_{1}}\left(\mathscr{S}_{1}, I_{1}\right) \rightarrow \mathcal{S} \mathcal{H}^{T_{2}}\left(\mathscr{S}_{2}, I_{2}\right)$. Il est également évident que le foncteur L $f^{\star}: \mathcal{S H}_{\mathrm{p}}^{T_{2}}\left(\mathscr{S}_{2}, I_{2}\right) \rightarrow \mathcal{S} \mathcal{H}_{\mathrm{p}}^{T_{1}}\left(\mathscr{S}_{1}, I_{1}\right)$ préserve les équivalences stables, ce foncteur induit donc un autre foncteur $\mathrm{L} f^{\star}: \mathcal{S} \mathcal{H}^{T_{2}}\left(\mathscr{S}_{2}, I_{2}\right) \rightarrow \mathcal{S} \mathcal{H}^{T_{1}}\left(\mathscr{S}_{1}, I_{1}\right)$ dont on vérifie facilement qu'il définit un adjoint à gauche à $\mathrm{R} f_{\star}$ (le fait que $\mathrm{R}_{\mathrm{p}} f_{\star}$ préserve les $\Omega$-spectres est essentiel ici). On obtient ainsi le théorème suivant :

THÉORÈme 2.11. - Soit $f:\left(\mathscr{S}_{1}, I_{1}, T_{1}\right) \rightarrow\left(\mathscr{S}_{2}, I_{2}, T_{2}\right)$ une application raisonnable de sites suspendus avec intervalles. On dispose de couples de foncteurs adjoints $\left(\mathrm{L} f^{\star}, \mathrm{R}_{\mathrm{p}} f_{\star}\right)$ entre les catégories $\mathcal{S \mathcal { H } _ { \mathrm { p } } ^ { T _ { k } }}\left(\mathscr{S}_{k}, I_{k}\right)$ et $\left(\mathrm{L} f^{\star}, \mathrm{R} f_{\star}\right)$ entre les catégories $\mathcal{S H}^{T_{k}}\left(\mathscr{S}_{k}, I_{k}\right)$ pour $k \in\{1,2\}$.

Proposition 2.12. - Soit $f:\left(\mathscr{S}_{1}, I_{1}, T_{1}\right) \rightarrow\left(\mathscr{S}_{2}, I_{2}, T_{2}\right)$ une application raisonnable de sites suspendus avec intervalles. Les diagrammes de catégories suivants sont commutatifs (à des isomorphismes canoniques de foncteurs près) pour tout entier naturel $n$ :

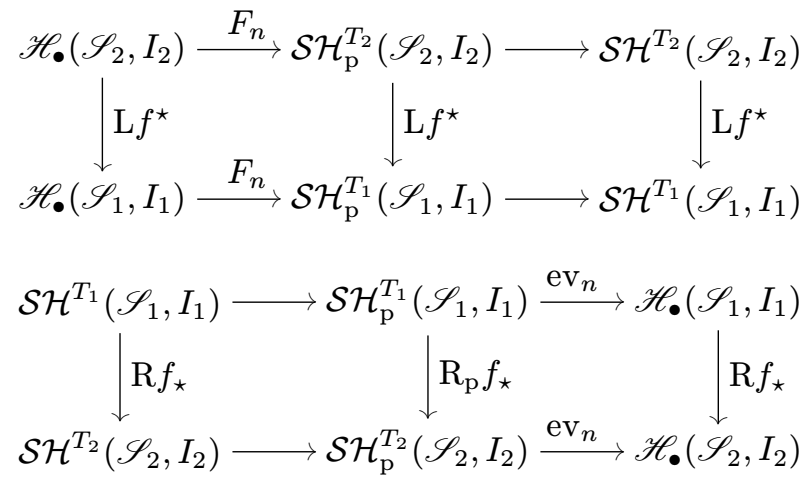


Tout d'abord, on a un couple de foncteurs adjoints $\left(\mathrm{L} f^{\star}, \mathrm{R} f_{\star}\right)$ entre les catégories $\mathscr{H}_{\bullet}\left(\mathscr{S}_{k}, I_{k}\right)$ (il s'agit d'une version pointée de la construction de [24, page 92], dont on a développé ici une version pour les spectres). Les foncteurs intervenant dans le premier diagramme sont des foncteurs dérivés à gauche des foncteurs évidents (ou des identités si la flèche n'est pas précisée) tandis que les foncteurs apparaissant sur le deuxième sont des foncteurs dérivés à droite; les deux diagrammes se correspondent par adjonction. Il est évident que le deuxième diagramme est commutatif (à des isomorphismes canoniques près), le premier l'est donc aussi.

Remarque 2.13. - Le lemme 2.8 (et ses variantes) montre qu'au niveau des catégories homotopiques (pointées ou non) et des catégories homotopiques projectives ou stables, les foncteurs $\mathrm{L} f^{\star}$ « commutent » aux colimites homotopiques.

Lemme 2.14. - Soit $f:\left(\mathscr{S}_{1}, I_{1}, T_{1}\right) \rightarrow\left(\mathscr{S}_{2}, I_{2}, T_{2}\right)$ une application raisonnable de sites suspendus avec intervalles. Le diagramme suivant commute à un isomorphisme canonique près :

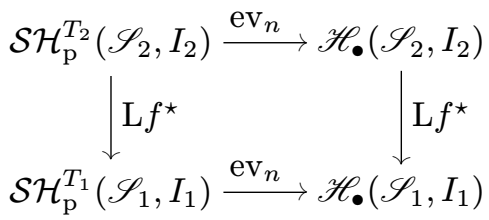

On construit facilement un morphisme canonique $\mathrm{L} f^{\star} \circ \mathrm{ev}_{n} \rightarrow \mathrm{ev}_{n} \circ \mathrm{L} f^{\star}$ de foncteurs $\mathcal{S H}_{\mathrm{p}}^{T_{2}}\left(\mathscr{S}_{2}, I_{2}\right) \rightarrow \mathscr{H}_{\bullet}\left(\mathscr{S}_{1}, I_{1}\right)$. Il s'agit de montrer que c'est un isomorphisme. Pour cela, il suffit de montrer que si $\mathbf{X}_{\mathrm{adm}}$ est la résolution admissible à gauche d'un objet $\mathbf{X} \in \mathbf{S p t}^{T_{2}}\left(\mathscr{S}_{2}\right)$ construite dans le lemme 2.6, l'objet $\mathrm{ev}_{n}\left(\mathbf{X}_{\text {adm }}\right)$ de $\mathbf{E s p} .\left(\mathscr{S}_{2}\right)$ est $f^{\star}$-admissible pour la version pointée de la définition de [24, remark 1.50, page 64]. En revenant à la construction spécifique de $\mathbf{X}_{\text {adm }}$, on voit qu'il suffit de montrer que si $X \in \mathscr{S}_{2}$ et $q \in \mathbf{N}$, alors $T_{2}^{\wedge q} \wedge X_{+}$ est un objet $f^{\star}$-admissible de Esp. $\left(\mathscr{S}_{2}\right)$, ce qui découle du lemme suivant :

Lemme 2.15. - Soit $f:\left(\mathscr{S}_{1}, I_{1}, T_{1}\right) \rightarrow\left(\mathscr{S}_{2}, I_{2}, T_{2}\right)$ une application raisonnable de sites suspendus avec intervalles. Soit $X$ un objet de Esp. $\left(\mathscr{S}_{2}\right)$. Si $X$ est $f^{\star}$-admissible, alors $T_{2} \wedge X$ aussi.

Un objet $X$ de Esp. $\left(\mathscr{S}_{2}\right)$ est $f^{\star}$-admissible si et seulement si pour tout objet $I_{1}$-fibrant $Y$ de Esp. $\left(\mathscr{S}_{1}\right)$ et toute $I_{2}$-équivalence faible $f_{\star} Y \rightarrow Y^{\prime}$ avec $Y^{\prime}$ un objet $I_{2}$-fibrant, la flèche évidente

$$
\operatorname{hom}\left(X, f_{\star} Y\right) \rightarrow \operatorname{hom}\left(X, Y^{\prime}\right)
$$

TOME $135-2007-\mathrm{N}^{\mathrm{O}} 4$ 
est une équivalence faible d'ensembles simpliciaux. On se donne donc un tel objet $X$, et on voudrait montrer que $T_{2} \wedge X$ est $f^{\star}$-admissible. On se donne alors un objet $I_{1}$-fibrant $Y$ de $\operatorname{Esp}_{\bullet}\left(\mathscr{S}_{1}\right)$, on choisit une $I_{2}$-équivalence faible $f_{\star} Y \rightarrow Y^{\prime}$ avec $Y^{\prime} I_{2}$-fibrant. On veut montrer que la flèche évidente

$$
\operatorname{hom}\left(T_{2} \wedge X, f_{\star} Y\right) \rightarrow \operatorname{hom}\left(T_{2} \wedge X, Y^{\prime}\right)
$$

Par adjonction, cela revient à montrer que la flèche

$$
\operatorname{hom}\left(X, \operatorname{Hom}_{\bullet}\left(T_{2}, f_{\star} Y\right)\right) \rightarrow \operatorname{hom}\left(X, \text { Hom }_{\bullet}\left(T_{2}, Y^{\prime}\right)\right)
$$

est une équivalence faible. Comme Hom. $\left(T_{2}, Y^{\prime}\right)$ est $I_{2}$-fibrant (puisque $Y^{\prime}$ l'est), la dernière condition donnée dans la définition d'une application raisonnable de sites suspendus avec intervalles implique que $\alpha: \mathbf{H o m}_{\bullet}\left(T_{2}, f_{\star} Y\right) \rightarrow$ Hom. $\left(T_{2}, Y^{\prime}\right)$ est une $I_{2}$-équivalence faible. On a un isomorphisme canonique Hom• $\left(T_{2}, f_{\star} Y\right)=f_{\star} \mathbf{H o m}_{\bullet}\left(T_{1}, Y\right)$; comme l'objet Hom• $\left(T_{1}, Y\right)$ est $I_{1}$-fibrant, on peut utiliser le fait que $X$ soit $f^{\star}$-admissible pour montrer que hom. $(X, \alpha)$ est une équivalence faible, ce qui est précisément ce qu'on voulait.

REMARQUE 2.16. - Le foncteur L $f^{\star}: \mathcal{S} \mathcal{H}_{\mathrm{p}}^{T_{2}}\left(\mathscr{S}_{2}, I_{2}\right) \rightarrow \mathcal{S} \mathcal{H}_{\mathrm{p}}^{T_{1}}\left(\mathscr{S}_{1}, I_{1}\right)$ ne préserve pas nécessairement les $\Omega$-spectres. On pourrait construire un contreexemple en considérant les «points réels » du $\mathbf{P}^{1}$-spectre BGL représentant la $K$-théorie algébrique (cf. [30, §6.2]). Pour obtenir la conclusion du lemme 2.14 pour le foncteur $\mathrm{L} f^{\star}: \mathcal{S} \mathcal{H}^{T_{2}}\left(\mathscr{S}_{2}, I_{2}\right) \rightarrow \mathcal{S} \mathcal{H}^{T_{1}}\left(\mathscr{S}_{1}, I_{1}\right)$, il convient de se limiter aux objets de $\mathcal{S} \mathcal{H}_{\mathrm{p}}^{T_{2}}\left(\mathscr{S}_{2}, I_{2}\right)$ qui sont des $\Omega$-spectres et qui le restent dans $\mathcal{S} \mathcal{H}_{\mathrm{p}}^{T_{1}}\left(\mathscr{S}_{1}, I_{1}\right)$ après application du foncteur $\mathrm{L} f^{\star}$.

Proposition 2.17. - On se donne deux applications raisonnables de sites suspendus avec intervalles $f:\left(\mathscr{S}_{1}, I_{1}, T_{1}\right) \rightarrow\left(\mathscr{S}_{2}, I_{2}, T_{2}\right)$ et $g:\left(\mathscr{S}_{2}, I_{2}, T_{2}\right) \rightarrow$ $\left(\mathscr{S}_{3}, I_{3}, T_{3}\right)$. Alors on peut définir une application raisonnable de sites suspendus avec intervalles $g \circ f:\left(\mathscr{S}_{1}, I_{1}, T_{1}\right) \rightarrow\left(\mathscr{S}_{3}, I_{3}, T_{3}\right)$, et on a des isomorphismes canoniques de foncteurs

$$
\begin{aligned}
\mathrm{R}_{\mathrm{p}}(g \circ f)_{\star} \stackrel{\sim}{\rightarrow} \mathrm{R}_{\mathrm{p}} g_{\star} \circ \mathrm{R}_{\mathrm{p}} f_{\star}: \mathcal{S} \mathcal{H}_{\mathrm{p}}^{T_{1}}\left(\mathscr{S}_{1}, I_{1}\right) \rightarrow \mathcal{S} \mathcal{H}_{\mathrm{p}}^{T_{3}}\left(\mathscr{S}_{3}, I_{3}\right), \\
\mathrm{R}(g \circ f)_{\star} \stackrel{\sim}{\rightarrow} \mathrm{R} g_{\star} \circ \mathrm{R} f_{\star}: \mathcal{S} \mathcal{H}^{T_{1}}\left(\mathscr{S}_{1}, I_{1}\right) \rightarrow \mathcal{S H} \mathcal{H}^{T_{3}}\left(\mathscr{S}_{3}, I_{3}\right), \\
\mathrm{L} f^{\star} \circ \mathrm{L} g^{\star} \stackrel{\sim}{\rightarrow} \mathrm{L}(g \circ f)^{\star}: \mathcal{S} \mathcal{H}_{\mathrm{p}}^{T_{3}}\left(\mathscr{S}_{3}, I_{3}\right) \rightarrow \mathcal{S H} \mathcal{H}_{\mathrm{p}}^{T_{1}}\left(\mathscr{S}_{1}, I_{1}\right), \\
\mathrm{L} f^{\star} \circ \mathrm{L} g^{\star} \stackrel{\sim}{\rightarrow} \mathrm{L}(g \circ f)^{\star}: \mathcal{S} \mathcal{H}^{T_{3}}\left(\mathscr{S}_{3}, I_{3}\right) \rightarrow \mathcal{S H} \mathcal{H}^{T_{1}}\left(\mathscr{S}_{1}, I_{1}\right) .
\end{aligned}
$$

Une fois que l'on sait que les applications continues raisonnables de sites (tout court) se composent, il n'y a pas de difficulté à construire l'application raisonnable de sites suspendus avec intervalles $g \circ f$. On peut ensuite montrer très facilement que les quatre morphismes de foncteurs considérés sont des isomorphismes. 
REMARQUe 2.18. - On peut ainsi parler des catégories bi-fibrées ${ }^{(5)}$ $(\mathscr{S}, I, T) \longmapsto \mathcal{S} \mathcal{H}_{\mathrm{p}}^{T}(\mathscr{S}, I)$ et $(\mathscr{S}, I, T) \longmapsto \mathcal{S H}^{T}(\mathscr{S}, I)$ au-dessus de la catégorie des sites suspendus avec intervalles (avec les applications raisonnables comme morphismes).

2.2. Changement de suspension. - Soit $(\mathscr{S}, I)$ un site avec intervalle. On se donne un morphisme $f: T^{\prime} \rightarrow T$ dans $\operatorname{Esp}_{\bullet}(\mathscr{S})$.

On définit un foncteur $f^{\star}: \mathbf{S p t}^{T}(\mathscr{S}) \rightarrow \mathbf{S} \mathbf{p t}^{T^{\prime}}(\mathscr{S})$ en faisant correspondre à un $T$-spectre $\mathbf{E}$ le $T^{\prime}$-spectre constitué de la même suite $\left(\mathbf{E}_{n}\right)_{n \in \mathbf{N}}$ d'objets de $\operatorname{Esp}_{\bullet}(\mathscr{S})$, les morphismes d'assemblage $\sigma_{n}^{\prime}$ étant définis par la composition suivante :

$$
T^{\prime} \wedge \mathbf{E}_{n} \stackrel{f \wedge \mathrm{id}_{\mathbf{E}_{n}}}{\longrightarrow} T \wedge \mathbf{E}_{n} \stackrel{\sigma_{n}}{\longrightarrow} \mathbf{E}_{n+1}
$$

Il est évident que $f^{\star}: \mathbf{S p t}^{T}(\mathscr{S}) \rightarrow \mathbf{S} \mathbf{S t}^{T^{\prime}}(\mathscr{S})$ préserve les $I$-équivalences projectives, on obtient ainsi un foncteur $f^{\star}: \mathcal{S H}_{\mathrm{p}}^{T}(\mathscr{S}, I) \rightarrow \mathcal{S} \mathcal{H}_{\mathrm{p}}^{T^{\prime}}(\mathscr{S}, I)$.

Proposition 2.19. - Soit $(\mathscr{S}, I)$ un site avec intervalle. Soit un morphisme $f: T^{\prime} \rightarrow T$ dans $\mathbf{E s p}_{\bullet}(\mathscr{S})$. Si $f$ est une I-équivalence faible, alors le foncteur $f^{\star}$ induit une équivalence de catégories $\mathcal{S H}_{\mathrm{p}}^{T}(\mathscr{S}, I) \rightarrow \mathcal{S H}_{\mathrm{p}}^{T^{\prime}}(\mathscr{S}, I)$.

Déduisons-en aussitôt le corollaire suivant :

Corollaire 2.20. - Soit $(\mathscr{S}, I)$ un site avec intervalle (possédant suffisamment de points). Soit un morphisme $f: T^{\prime} \rightarrow T$ dans $\mathbf{E s p}_{\bullet}(\mathscr{S})$. Si $f$ est une I-équivalence faible, alors le foncteur $f^{\star}: \mathcal{S H}^{T}(\mathscr{S}, I) \rightarrow \mathcal{S H}^{T^{\prime}}(\mathscr{S}, I)$ est une équivalence de catégories.

D'après la proposition, $f^{\star}: \mathcal{S} \mathcal{H}_{\mathrm{p}}^{T}(\mathscr{S}, I) \rightarrow \mathcal{S H}_{\mathrm{p}}^{T^{\prime}}(\mathscr{S}, I)$ est une équivalence de catégories. Comme $f: T \rightarrow T^{\prime}$ est une $I$-équivalence faible, il est évident que si $\mathbf{E}$ est un objet de $\mathcal{S} \mathcal{H}^{T}(\mathscr{S}, I)$, alors $\mathbf{E}$ est un $\Omega$-spectre si et seulement si $f^{\star} \mathbf{E}$ est un $\Omega$-spectre. On en déduit qu'un morphisme $g: \mathbf{E} \rightarrow \mathbf{E}^{\prime}$ dans $\mathcal{S H}_{\mathrm{p}}^{T}(\mathscr{S}, I)$ est une $I$-équivalence stable si et seulement si $f^{\star}(g): f^{\star} \mathbf{E} \rightarrow f^{\star} \mathbf{E}^{\prime}$ est une $I$-équivalence stable, ce qui permet de conclure.

Pour démontrer la proposition, nous allons construire un foncteur adjoint à droite $f_{\star}: \mathbf{S p t}^{T^{\prime}}(\mathscr{S}) \rightarrow \mathbf{S} p t^{T}(\mathscr{S})$ de $f^{\star}: \mathbf{S p t}^{T}(\mathscr{S}) \rightarrow \mathbf{S p t}^{T^{\prime}}(\mathscr{S})$.

Soit $\mathbf{E}$ un objet de $\mathbf{S p t}^{T^{\prime}}(\mathscr{S})$. On note $f_{\star} \mathbf{E}_{n}$ l'égalisateur des deux flèches

$$
\alpha, \beta: \prod_{i \in \mathbf{N}} \mathbf{H o m}_{\bullet}\left(T^{\wedge i}, \mathbf{E}_{n+i}\right) \rightarrow \prod_{i \in \mathbf{N}} \mathbf{H o m}_{\bullet}\left(T^{\prime} \wedge T^{\wedge i}, \mathbf{E}_{n+i+1}\right)
$$

où $\alpha$ est induite par les flèches

$$
\mathbf{H o m}_{\bullet}\left(T^{\wedge i}, \mathbf{E}_{n+i}\right) \rightarrow \mathbf{H o m}_{\bullet}\left(T^{\prime} \wedge T^{\wedge i}, \mathbf{E}_{n+i+1}\right)
$$

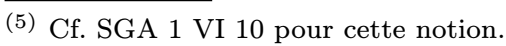

TOME $135-2007-\mathrm{N}^{\mathrm{O}} 4$ 
induites par les morphismes d'assemblage de $\mathbf{E}$ et où $\beta$ est induite par les flèches

$$
\mathbf{H o m}_{\bullet}\left(T^{\wedge 1+i}, \mathbf{E}_{n+i+1}\right) \rightarrow \mathbf{H o m}_{\bullet}\left(T^{\prime} \wedge T^{\wedge i}, \mathbf{E}_{n+i+1}\right)
$$

provenant de la composition à droite avec $f \wedge \operatorname{id}_{T^{\wedge i}}: T^{\prime} \wedge T^{\wedge i} \rightarrow T \wedge T^{\wedge i}=T^{\wedge 1+i}$.

On note $p_{i, n}$ les morphismes évidents :

$$
p_{i, n}:\left(f_{\star} \mathbf{E}\right)_{n} \rightarrow \mathbf{H o m}_{\bullet}\left(T^{\wedge i}, \mathbf{E}_{n+i}\right) .
$$

Pour tout entier naturel $n$, on définit le morphisme d'assemblage $T \wedge f_{\star} \mathbf{E}_{n} \rightarrow$ $f_{\star} \mathbf{E}_{n+1}$ de façon à rendre commutatif le diagramme suivant :

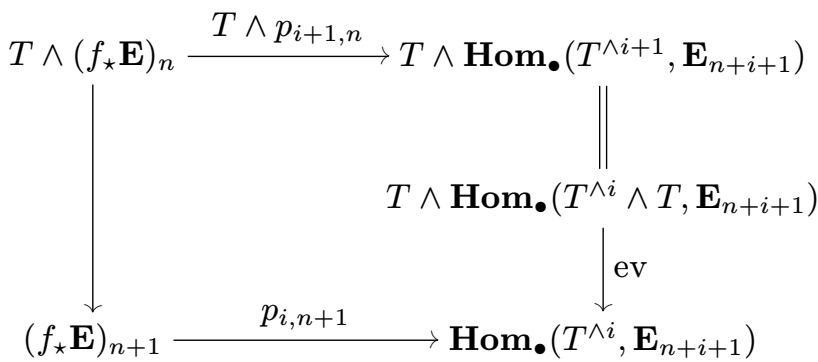

On montre très facilement le lemme suivant :

LEMme 2.21. - Les foncteurs $f^{\star}: \mathbf{S p t}^{T}(\mathscr{S}) \rightarrow \mathbf{S} \mathbf{p t}^{T^{\prime}}(\mathscr{S})$ et $f_{\star}: \mathbf{S p t}^{T^{\prime}}(\mathscr{S}) \rightarrow$ $\mathbf{S p t}^{T}(\mathscr{S})$ forment un couple $\left(f^{\star}, f_{\star}\right)$ de foncteurs adjoints.

Pour tout morphisme $f: T^{\prime} \rightarrow T$ dans $\operatorname{Esp}_{\bullet}(\mathscr{S})$, il est clair que $f^{\star}: \mathbf{S p t}^{T}(\mathscr{S}) \rightarrow \mathbf{S p t}^{T^{\prime}}(\mathscr{S})$ préserve les monomorphismes et les $I$-équivalences projectives. On a ainsi une adjonction de Quillen (pour les structures injectives) ; on peut appliquer [13, lemma 7.9, Chapter II] et [13, theorem 7.7, Chapter II] pour montrer que $f^{\star}: \mathcal{S H}_{\mathrm{p}}^{T}(\mathscr{S}, I) \rightarrow \mathcal{S H}_{\mathrm{p}}^{T^{\prime}}(\mathscr{S}, I)$ admet un adjoint à droite $\mathrm{R} f_{\star}: \mathcal{S} \mathcal{H}_{\mathrm{p}}^{T^{\prime}}(\mathscr{S}, I) \rightarrow \mathcal{S} \mathcal{H}_{\mathrm{p}}^{T}(\mathscr{S}, I)$.

Nous sommes maintenant en mesure d'établir la proposition 2.19. Tout d'abord, on peut supposer que $f: T^{\prime} \rightarrow T$ est un monomorphisme; en effet, d'après [13, lemma 8.4, Chapter II], comme tous les objets de Esp.(S) sont cofibrants, il existe une factorisation $f=g \circ j$ où $j$ est une cofibration (triviale) et $q$ un inverse à gauche d'une cofibration triviale.

On suppose donc que $f$ est une $I$-équivalence faible qui est aussi un monomorphisme. On veut montrer que les foncteurs adjoints $f^{\star}$ et $\mathrm{R} f_{\star}$ définissent des équivalences de catégories inverses l'une de l'autre entre les catégories $\mathcal{S} \mathcal{H}_{\mathrm{p}}^{T}(\mathscr{S}, I)$ et $\mathcal{S H}_{\mathrm{p}}^{T^{\prime}}(\mathscr{S}, I)$. D'après [13, corollary 7.8, Chapter II], il s'agit de montrer que si on a une flèche $g: f^{\star} \mathbf{F} \rightarrow \mathbf{E}$ dans $\mathbf{S p t}^{T^{\prime}}(\mathscr{S})$ avec $\mathbf{F}$ un objet de $\mathbf{S p t}^{T}(\mathscr{S})$ et $\mathbf{E}$ un $T^{\prime}$-spectre injectivement $I$-fibrant, alors $g$ est une $I$-équivalence projective si et seulement si l'application adjointe $\mathbf{F} \rightarrow f_{\star} \mathbf{E}$ est 
une $I$-équivalence projective. On voit aussitôt que pour établir cela, il suffit de montrer que si $\mathbf{E}$ est un $T^{\prime}$-spectre injectivement $I$-fibrant, le morphisme d'adjonction $f^{\star} f_{\star} \mathbf{E} \rightarrow \mathbf{E}$ est une $I$-équivalence projective, autrement dit que les morphismes évidents $p_{0, n}:\left(f_{\star} \mathbf{E}\right)_{n} \rightarrow \mathbf{E}_{n}$ sont des $I$-équivalences faibles. Pour établir ce fait, on récrit $\left(f_{\star} \mathbf{E}\right)_{n}$ comme la limite projective du diagramme suivant :

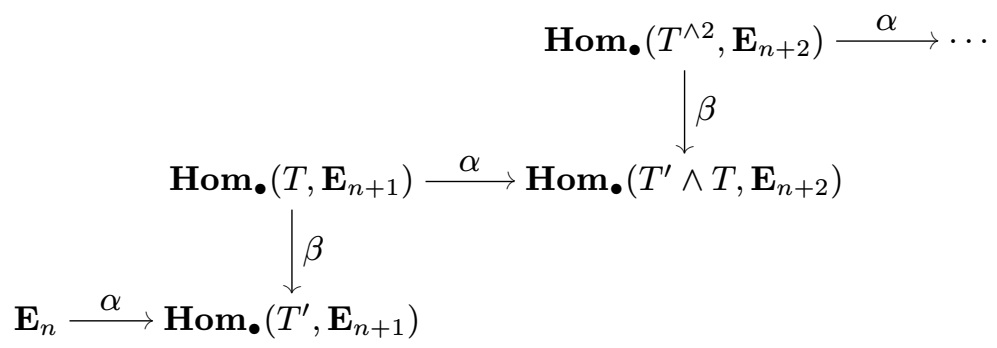

On a supposé ici que $f: T^{\prime} \rightarrow T$ était une $I$-cofibration triviale, les flèches verticales (notées $\beta$ ) sont donc des $I$-fibrations triviales (le $T^{\prime}$-spectre $\mathbf{E}$ étant supposé projectivement $I$-fibrant). En complétant le diagramme avec des carrés cartésiens, on peut observer que $\left(f_{\star} \mathbf{E}\right)_{n}$ s'identifie à la limite projective d'une tour de $I$-fibrations triviales dont la base est $\mathbf{E}_{n}$; la flèche $\left(f_{\star} \mathbf{E}\right)_{n} \rightarrow \mathbf{E}_{n}$ est donc une $I$-fibration triviale, ce qui montre que le morphisme d'adjonction $f^{\star} f_{\star} \mathbf{E} \rightarrow \mathbf{E}$ est une $I$-équivalence projective et achève la démonstration de la proposition.

Proposition 2.22. - Soit $(\mathscr{S}, I)$ un site avec intervalle. Soit $T$ un objet de Esp.(S). Alors, on a un foncteur $\mathbf{S p t}^{T}(\mathscr{S}) \rightarrow \mathbf{S p t}{ }^{T \wedge T}(\mathscr{S})$ induisant une équivalence de catégories $\mathcal{S H}^{T}(\mathscr{S}, I) \rightarrow \mathcal{S H}^{T \wedge T}(\mathscr{S}, I)$.

On définit un foncteur $G: \mathbf{S p t}^{T}(\mathscr{S}) \rightarrow \mathbf{S p t}^{T \wedge T}(\mathscr{S})$ de la manière suivante. Soit $\mathbf{E}$ un objet de $\mathbf{S p t}^{T}(\mathscr{S})$. On définit un $T \wedge T$-spectre $G \mathbf{E}$ en posant $(G \mathbf{E})_{n}=\mathbf{E}_{2 n}$ et en définissant le morphisme d'assemblage par la composition suivante :

$$
T \wedge T \wedge \mathbf{E}_{2 n} \stackrel{T \wedge \sigma_{2 n}}{\longrightarrow} T \wedge \mathbf{E}_{2 n+1} \stackrel{\sigma_{2 n+1}}{\longrightarrow} \mathbf{E}_{2 n+2} .
$$

On définit un foncteur $F: \mathbf{S} \mathbf{p t}^{T \wedge T}(\mathscr{S}) \rightarrow \mathbf{S p t}^{T}(\mathscr{S})$ en posant pour tout $T \wedge T$ spectre $\mathbf{F}$ et tout entier naturel $n$ :

$$
(F \mathbf{F})_{2 n}=\mathbf{F}_{n} \quad(F \mathbf{F})_{2 n+1}=\operatorname{Hom}_{\bullet}\left(T, \mathbf{F}_{n+1}\right)
$$

Pour tout entier naturel $n$, le morphisme d'assemblage $T \wedge(F \mathbf{F})_{2 n+1} \rightarrow$ $(F \mathbf{F})_{2 n+2}$ n'est autre que le morphisme d'évaluation $T \wedge \mathbf{H o m}_{\bullet}\left(T, \mathbf{F}_{n+1}\right) \rightarrow$ $\mathbf{F}_{n+1}$ tandis que le morphisme d'assemblage $T \wedge(F \mathbf{F})_{2 n} \rightarrow(F \mathbf{F})_{2 n+1}$ est le morphisme $T \wedge \mathbf{F}_{n} \rightarrow \mathbf{H o m} .\left(T, \mathbf{F}_{n+1}\right)$ adjoint du morphisme $T \wedge\left(T \wedge \mathbf{F}_{n}\right) \rightarrow \mathbf{F}_{n+1}$ que l'on identifie à un morphisme d'assemblage du $T \wedge T$-spectre $\mathbf{F}$. 
Lemme 2.23. - Les foncteurs $G: \mathbf{S p t}^{T}(\mathscr{S}) \rightarrow \mathbf{S p t} \mathbf{t}^{T \wedge T}(\mathscr{S})$ et $F: \mathbf{S p t}^{T \wedge T}(\mathscr{S}) \rightarrow \mathbf{S p t}^{T}(\mathscr{S})$ définissent un couple de foncteurs adjoints $(G, F)$.

\section{C'est évident.}

Il est clair que le foncteur $G: \mathbf{S p t}^{T}(\mathscr{S}) \rightarrow \mathbf{S} \mathbf{p} \mathbf{t}^{T \wedge T}(\mathscr{S})$ préserve les monomorphismes et les $I$-équivalences projectives; de plus $F$ préserve les $I$-équivalences projectives entre objets projectivement $I$-fibrants. On en déduit une adjonction de Quillen au niveau des structures injectives, d'où des foncteurs $G: S \mathcal{H}_{\mathrm{p}}^{T}(\mathscr{S}, I) \rightarrow \mathcal{S} \mathcal{H}_{\mathrm{p}}^{T \wedge T}(\mathscr{S}, I)$ et $\mathrm{R}_{\mathrm{p}} F: \mathcal{S} \mathcal{H}_{\mathrm{p}}^{T \wedge T}(\mathscr{S}, I) \rightarrow \mathcal{S H}_{\mathrm{p}}^{T}(\mathscr{S}, I)$ formant un couple de foncteurs adjoints $\left(G, \mathrm{R}_{\mathrm{p}} F\right)$. Maintenant, si $\mathbf{F}$ est un $T \wedge T$-spectre projectivement $I$-fibrant, alors $\mathbf{F}$ est un $\Omega$-spectre si et seulement si $F \mathbf{F}$ est un $\Omega$-spectre, on en déduit que le foncteur $G: \mathcal{S H}_{\mathrm{p}}^{T}(\mathscr{S}, I) \rightarrow \mathcal{S H}_{\mathrm{p}}^{T \wedge T}(\mathscr{S}, I)$ préserve les équivalences stables, d'où un foncteur induit $G: \mathcal{S} \mathcal{H}^{T}(\mathscr{S}, I) \rightarrow \mathcal{S H}^{T \wedge T}(\mathscr{S}, I)$ et une adjonction de Quillen au niveau des structures stables injectives, ce dernier foncteur admet donc un adjoint à droite $\mathrm{RF}: \mathcal{S H}^{T \wedge T}(\mathscr{S}, I) \rightarrow \mathcal{S H}^{T}(\mathscr{S}, I)$. On dispose de transformations naturelles $\operatorname{id}_{\mathcal{S H}} \mathcal{H}^{T}(\mathscr{S}, I) \rightarrow \mathrm{RF} \circ G: \mathcal{S H}^{T}(\mathscr{S}, I) \rightarrow \mathcal{S H}^{T}(\mathscr{S}, I)$ et $G \circ \mathrm{R} F \rightarrow \operatorname{id}_{\mathcal{S H} \mathcal{H}^{T \wedge T}(\mathscr{S}, I)}: \mathcal{S} \mathcal{H}^{T \wedge T}(\mathscr{S}, I) \rightarrow \mathcal{S H}^{T \wedge T}(\mathscr{S}, I)$ dont on vérifie trivialement que ce sont des isomorphismes. On a donc établi la proposition.

\section{Structure triangulée, $T$-espaces de lacets}

Nous allons voir que sous certaines hypothèses, les catégories homotopiques stables construites ici sont canoniquement munies de structures triangulées (cf. théorème 3.10). Nous étudierons ensuite les $T$-espaces de lacets, ceux-ci ayant quelque rapport avec la structure triangulée; cette étude conduira à la comparaison de la notion d'équivalence stable définie ici et celle donnée dans [20] (cf. théorème 3.35).

\section{1. Énoncés généraux}

DÉfinition 3.1. - Soit $\mathscr{H}$ une catégorie et $\Sigma: \mathscr{H} \rightarrow \mathscr{H}$ un foncteur. On note $\mathscr{H}\left[\Sigma^{-1}\right]$ la catégorie dont les objets sont les couples $(X, n)$ où $X$ est un objet de $\mathscr{H}$ et $n$ un entier relatif, un morphisme $(X, n) \rightarrow(Y, m)$ dans $\mathscr{H}\left[\Sigma^{-1}\right]$ est un élément de la limite inductive

$$
\underset{r \geq \max (-n,-m)}{\operatorname{colim}} \operatorname{Hom}_{\mathscr{H}}\left(\Sigma^{r+n} X, \Sigma^{r+m} Y\right)
$$

où les applications de transition sont induites par le foncteur $\Sigma$, la composition des flèches est définie de façon évidente. 
On a un foncteur évident $s: \mathscr{H} \rightarrow \mathscr{H}\left[\Sigma^{-1}\right]$ envoyant $X$ sur $(X, 0)$. On note

$$
-[1]: \mathscr{H}\left[\Sigma^{-1}\right] \rightarrow \mathscr{H}\left[\Sigma^{-1}\right]
$$

le foncteur qui à un objet $(X, n)$ associe $(X, n+1)$, la définition de ce foncteur au niveau des morphismes étant évidente. Ce foncteur - [1] est un automorphisme de la catégorie $\mathscr{H}\left[\Sigma^{-1}\right]$, on note $-[n]$ ses puissances, pour tout entier relatif $n$. On a un isomorphisme canonique de foncteurs $\mathscr{H} \rightarrow \mathscr{H}\left[\Sigma^{-1}\right]$ entre $s \circ \Sigma$ et [1] $s$ (autrement dit, on a un isomorphisme naturel $(X, 1) \cong(\Sigma X, 0)$ pour tout objet $X$ de $\mathscr{H})$.

L'énoncé suivant peut être dégagé à partir de la construction indiquée par Voevodsky dans $[30, \S 4]$ :

ThÉORÈme 3.2. - Soit $\mathscr{C}$ une catégorie de modèles pointée ${ }^{(6)}$. Notons $\mathscr{H}$ sa catégorie homotopique et $\Sigma: \mathscr{H} \rightarrow \mathscr{H}$ le foncteur de suspension (cf. [25, $\S 2$, Chapter I]). Alors, la catégorie $\mathscr{H}\left[\Sigma^{-1}\right]$ est canoniquement munie d'une structure de catégorie triangulée.

On trouve presque cet énoncé dans [25, proposition 5 , §3, Chapter I]; je me contenterai ici d'esquisser la construction dans le cas plus aisé où $\mathscr{C}$ est de plus supposée simpliciale. On dispose ainsi d'un foncteur $\otimes: \mathscr{C} \times \boldsymbol{\Delta}^{\mathrm{opp}} \mathbf{E n s} \rightarrow$ $\mathscr{C}$. Notons d'abord que si $(K, k)$ est un ensemble simplicial pointé, on peut noter $X \wedge(K, k)$ le quotient $X \otimes K / X \otimes k$, ce qui définit un foncteur $\wedge: \mathscr{C} \times$ $\boldsymbol{\Delta}^{\mathrm{opp}}$ Ens. $\rightarrow \mathscr{C}$. On peut identifier $\Sigma$ au foncteur dérivé à gauche de $-\wedge$ $S^{1}: \mathscr{C} \rightarrow \mathscr{C}$

Soit $f: X \rightarrow Y$ un morphisme dans $\mathscr{C}$ entre objets cofibrants. On définit cône $(f)$ de façon à avoir un carré cocartésien dans $\mathscr{C}$ :

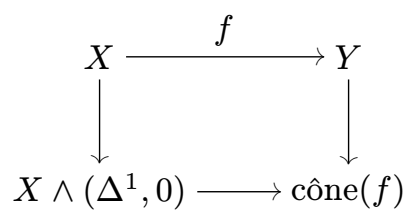

où la flèche $X \rightarrow X \wedge\left(\Delta^{1}, 0\right)$, identifiée à $X \wedge S^{0} \rightarrow X \wedge\left(\Delta^{1}, 0\right)$ provient de l'inclusion $S^{0} \rightarrow\left(\Delta^{1}, 0\right)$.

On a un morphisme évident cône $(f) \rightarrow \operatorname{cône}(X \rightarrow \bullet)=X \wedge S^{1}$. On a ainsi défini un triangle (à valeurs dans la catégorie $\mathscr{C}\left[\Sigma^{-1}\right]$ munie du foncteur de translation $-[1])$ :

$$
(X, 0) \stackrel{f}{\rightarrow}(Y, 0) \rightarrow(\text { cône }(f), 0) \rightarrow(X, 1) ;
$$

notons $\mathscr{T}_{f}$ ce triangle.

$\overline{(6)}$ Une catégorie de modèles est dite pointée si elle possède un objet nul.

TOME $135-2007-\mathrm{N}^{\mathrm{O}} 4$ 
Soit $\mathscr{T}=(A \stackrel{u}{\rightarrow} B \stackrel{v}{\rightarrow} C \stackrel{w}{\longrightarrow} A[1])$ un triangle, pour tout entier relatif $n$, on note $\mathscr{T}[n]$ le triangle

$$
A[n] \stackrel{(-1)^{n} u}{\longrightarrow} B[n] \stackrel{(-1)^{n} v}{\longrightarrow} C[n] \stackrel{(-1)^{n} w}{\longrightarrow} A[n+1] .
$$

DÉFInition 3.3. - On dit d'un triangle de $\mathscr{C}\left[\Sigma^{-1}\right]$ qu'il est distingué s'il est isomorphe à un triangle de la forme $\mathscr{T}_{f}[n]$ pour un certain entier relatif $n$ et un morphisme $f$ entre objets cofibrants de $\mathscr{C}$.

Dans ce cadre simplicial, la vérification des axiomes des catégories triangulées est vraiment facile; pour le cas général, cf. [25, proposition $5, \S 3$, Chapter I].

Corollaire 3.4. - Soit $\mathscr{C}$ une catégorie de modèles pointée, soit $\mathscr{H}$ sa catégorie homotopique. On suppose que le foncteur de suspension $\Sigma: \mathscr{H} \rightarrow \mathscr{H}$ est une autoéquivalence. Alors $\mathscr{H}$ est canoniquement munie d'une structure de catégorie triangulée.

En effet, dans ce cas, le foncteur évident $s: \mathscr{H} \rightarrow \mathscr{H}\left[\Sigma^{-1}\right]$ est une équivalence de catégories. Stricto sensu, $\mathscr{H}$ n'est pas une catégorie triangulée au sens de [29, définition 1.1.1, Chapitre II] puisque $\Sigma$ n'a aucune raison d'être un automorphisme de la catégorie $\mathscr{H}$ : ce n'est qu'une autoéquivalence. On laisse en exercice au lecteur de se convaincre qu'il ne s'agit pas là d'un problème très sérieux (au pire, on remplace $\mathscr{H}$ par la catégorie $\mathscr{H}\left[\Sigma^{-1}\right]$ qui lui est équivalente).

Dans la suite, si $\mathscr{C}$ est une catégorie de modèles pointée satisfaisant les hypothèses de ce corollaire, on munira toujours la catégorie homotopique de $\mathscr{C}$ de la structure triangulée donnée par ce corollaire.

REMARQUE 3.5. - On peut interpréter le théorème 3.2 à la lumière de la théorie des dérivateurs initiée par A. Grothendieck. D'après [9], on sait qu'à une catégorie de modèles $\mathscr{C}$ satisfaisant certaines conditions techniques supplémentaires, on peut associer un dérivateur $\mathbf{D}_{\mathscr{C}}$ : on considère simultanément les catégories $\mathbf{D}_{\mathscr{C}}(\mathscr{I})$ pour toute petite catégorie $\mathscr{I}$ (et leur fonctorialité), où $\mathbf{D}_{\mathscr{C}}(\mathscr{I})$ est ici la catégorie homotopique de la catégorie des foncteurs $\mathscr{I} \rightarrow \mathscr{C}$ munie d'une structure de catégorie de modèles convenable. Dans [23], G. Maltsiniotis définit une structure triangulée sur $\mathbf{D}(\bullet)$ si $\mathbf{D}$ est un dérivateur satisfaisant certaines conditions, ainsi, le théorème 3.2 peut presqu'être vu comme un cas particulier de cette construction au niveau des dérivateurs.

REMARQUE 3.6. - La construction du théorème 3.2 étant valable pour toute catégorie de modèles pointée, on peut aussi l'appliquer à la catégorie opposée $\mathscr{C}^{\text {opp }}$ de la catégorie de modèles $\mathscr{C}$ de départ. Le foncteur de suspension sur sa catégorie homotopique $\mathscr{H}^{\text {opp }}$ est induit par le foncteur « espace de lacets » $\Omega: \mathscr{H} \rightarrow \mathscr{H}$ (adjoint à droite du foncteur $\Sigma: \mathscr{H} \rightarrow \mathscr{H}$ ). On dispose donc aussi 
d'une structure triangulée sur $\mathscr{H}\left[\Omega^{-1}\right]^{\text {opp }}$ (et donc aussi sur $\mathscr{H}\left[\Omega^{-1}\right]$, cf. [29, $\S 1.1 .7$, Chapitre II]). Maintenant, si on suppose que $\Sigma$ (et donc aussi $\Omega$ ) est une autoéquivalence de la catégorie $\mathscr{H}$, alors les structures triangulées obtenues sur $\mathscr{H}\left[\Sigma^{-1}\right]$ et $\mathscr{H}\left[\Omega^{-1}\right]$ donnent a priori deux structures triangulées sur la catégorie $\mathscr{H}$. On peut montrer que ces deux structures triangulées coïncident (cf. [22]).

REMARque 3.7. - La remarque précédente n'est pas complètement anodine. Si on dispose d'un foncteur $F: \mathscr{C} \rightarrow \mathscr{D}$ entre catégories de modèles, il est fréquent qu'on sache montrer facilement que $F$ « commute » aux foncteurs de suspension et que le foncteur induit $\mathscr{C}\left[\Sigma^{-1}\right] \rightarrow \mathscr{D}\left[\Sigma^{-1}\right]$ préserve les suites « cofibrées » (et donc les triangles distingués), autrement dit $F$ va induire un foncteur triangulé. Parfois, la propriété précédente ne se voit pas à l'œil nu et on obtient plus facilement la propriété duale, à savoir, la compatibilité de $F$ avec les foncteurs « espace de lacets » et la préservation des «suites fibrées ». La remarque précédente réconcilie ces deux approches.

La proposition suivante montre que les catégories de modèles pointées dont la catégorie homotopique est naturellement munie d'une structure de catégorie triangulée sont stables par localisation à la Bousfield :

Proposition 3.8. - Soit $\mathscr{C}$ une catégorie de modèles pointée. Soit $\mathscr{S}$ une famille de morphismes dans $\mathscr{C}$. On suppose que la catégorie de modèles localisée à la Bousfield à droite $\mathscr{C}_{\mathscr{S}}$ de $\mathscr{C}$ par rapport à $\mathscr{S}$ existe. Si le foncteur de suspension $\Sigma: \mathscr{H}(\mathscr{C}) \rightarrow \mathscr{H}(\mathscr{C})$ est une équivalence de catégories, alors le foncteur de suspension sur $\mathscr{H}\left(\mathscr{C}_{\mathscr{S}}\right)$ aussi.

Notons $\mathscr{W}$ la famille des morphismes dans $\mathscr{H}(\mathscr{C})$ qui sont des équivalences $\mathscr{S}$-locales. La foncteur évident $\mathscr{H}(\mathscr{C}) \rightarrow \mathscr{H}\left(\mathscr{C}_{\mathscr{S}}\right)$ identifie $\mathscr{H}\left(\mathscr{C}_{\mathscr{S}}\right)$ à la catégorie $\mathscr{H}(\mathscr{C})\left[\mathscr{W}^{-1}\right]$. On dispose d'un morphisme $j_{X}: X \rightarrow R X$ appartenant à $\mathscr{W}$ avec $R X \mathscr{S}$-local, et ce fonctoriellement en $X \in \mathscr{H}(\mathscr{C})$.

Le foncteur de suspension $\Sigma: \mathscr{H}(\mathscr{C}) \rightarrow \mathscr{H}(\mathscr{C})$ préserve les équivalences $\mathscr{S}$ locales, il induit le foncteur de suspension $\Sigma_{\mathscr{S}}: \mathscr{H}(\mathscr{C})\left[\mathscr{W}^{-1}\right] \rightarrow \mathscr{H}(\mathscr{C})\left[\mathscr{W}^{-1}\right]$. Notons $\Omega: \mathscr{H}(\mathscr{C}) \rightarrow \mathscr{H}(\mathscr{C})$ le foncteur espace de lacets, adjoint à droite de $\Sigma$. On peut considérer le foncteur $\mathscr{H}(\mathscr{C}) \rightarrow \mathscr{H}(\mathscr{C})\left[\mathscr{W}^{-1}\right]$ qui à $X$ associe $\Omega R X$, il envoie les morphismes de $\mathscr{W}$ sur des isomorphismes, induisant donc un foncteur $\Omega_{\mathscr{S}}: \mathscr{H}(\mathscr{C})\left[\mathscr{W}^{-1}\right] \rightarrow \mathscr{H}(\mathscr{C})\left[\mathscr{W}^{-1}\right]$ qui s'identifie au foncteur espace de lacets sur la catégorie homotopique de $\mathscr{C}_{\mathscr{S}}$. Par hypothèse, les morphismes d'adjonction $\Sigma \Omega \rightarrow \operatorname{id}_{\mathscr{H}(\mathscr{C})}$ et $\mathrm{id}_{\mathscr{H}(\mathscr{C})} \rightarrow \Omega \Sigma$ sont des isomorphismes, il s'agit de montrer qu'il en va de même pour le couple de foncteurs adjoints $\left(\Sigma_{\mathscr{S}}, \Omega_{\mathscr{S}}\right)$.

TOME $135-2007-\mathrm{N}^{\mathrm{O}} 4$ 
Pour tout objet $X$ de $\mathscr{H}(\mathscr{C})\left[\mathscr{W}^{-1}\right]$, le morphisme d'adjonction $\Sigma_{\mathscr{S}} \Omega_{\mathscr{S}} X \rightarrow$ $X$ est donné par le zigzag suivant où la flèche à contresens est bien dans $\mathscr{W}$ :

$$
\Sigma \Omega(R X) \stackrel{\text { ad }}{\longrightarrow} R X \stackrel{j_{X}}{\leftarrow} X .
$$

Le morphisme d'adjonction $\Sigma_{\mathscr{S}} \Omega_{\mathscr{C}} \rightarrow \operatorname{id}_{\mathscr{H}(\mathscr{C})\left[\mathscr{W}^{-1}\right]}$ est donc un isomorphisme. Concernant l'autre morphisme d'adjonction, pour tout objet $X \in \mathscr{H}(\mathscr{C})\left[\mathscr{W}^{-1}\right]$, il est donné par la composition suivante :

$$
X \stackrel{\text { ad }}{\longrightarrow} \Omega \Sigma X \stackrel{j_{\Omega \Sigma X}}{\longrightarrow} R(\Omega \Sigma X) .
$$

Par hypothèse, le morphisme de gauche est un isomorphisme, celui de droite est dans $\mathscr{W}$; le morphisme composé est donc un isomorphisme dans la catégorie $\mathscr{H}(\mathscr{C})\left[\mathscr{W}^{-1}\right]$, ce qui achève la démonstration de cette proposition.

REMARQUE 3.9. - Sous les hypothèses de la proposition 3.8, la structure triangulée sur $\mathscr{H}\left(\mathscr{C}_{\mathscr{S}}\right)$ coïncide bien entendu avec la structure triangulée obtenue par localisation (cf. [29, théorème 2.2.6, Chapitre II]) à partir de celle sur $\mathscr{H}(\mathscr{S})$.

3.2. Catégories $\mathcal{S} \mathcal{H}^{T}(\mathscr{S}, I)$. - On va donner ici une condition suffisante pour que la catégorie homotopique stable d'un site suspendu avec intervalle soit triangulée :

THÉORÈme 3.10. - Soit $(\mathscr{S}, I, T)$ un site suspendu avec intervalle. On suppose qu'il existe un isomorphisme $T \simeq S^{1} \wedge T^{\prime}$ dans $\mathscr{H}_{\bullet}(\mathscr{S}, I)$. Alors, le foncteur $-\wedge S^{1}: \mathbf{S p t}^{T}(\mathscr{S}) \rightarrow \mathbf{S p t}^{T}(\mathscr{S})$ induit une équivalence de catégories $\mathcal{S} \mathcal{H}^{T}(\mathscr{S}, I) \rightarrow \mathcal{S H}^{T}(\mathscr{S}, I)$. La catégorie $\mathcal{S H}^{T}(\mathscr{S}, I)$ est donc canoniquement munie d'une structure triangulée (cf. corollaire 3.4).

Lemme 3.11. - Soit $(\mathscr{S}, I, A)$ un site suspendu avec intervalle. Si le foncteur de suspension sur $\mathcal{S H}^{A}(\mathscr{S}, I)$ est une équivalence de catégories, alors c'est aussi le cas dans $\mathcal{S H}^{A \wedge B}(\mathscr{S}, I)$ pour tout $B \in \mathbf{E s p}_{\bullet}(\mathscr{S})$.

On peut définir une notion de $(A, B)$-bispectre : un tel objet consiste en la donnée d'une famille $\left(\mathscr{B}_{i, j}\right)_{(i, j) \in \mathbf{N}^{2}}$ d'objets de $\mathbf{E s p}_{\bullet}(\mathscr{S})$ munis de morphismes d'assemblage horizontaux $\sigma_{\mathrm{h}}: A \wedge \mathscr{B}_{i, j} \rightarrow \mathscr{B}_{i, j+1}$ et verticaux $\sigma_{\mathrm{v}}: B \wedge \mathscr{B}_{i, j} \rightarrow$ $\mathscr{B}_{i+1, j}$ rendant commutatifs les diagrammes évidents :

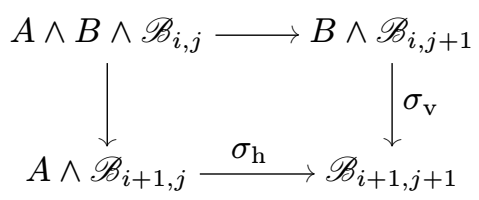

BULletin DE LA SOCiÉtÉ MATHÉmATiQUE DE FRANCE 
La notion de morphisme de $(A, B)$-bispectre est évidente, on obtient ainsi une catégorie $\operatorname{Bispt}^{A, B}(\mathscr{S})$. La notion de diagonale d'un bispectre donne un foncteur $\Delta: \mathbf{B i s p t}^{A, B}(\mathscr{S}) \rightarrow \mathbf{S p t}^{A \wedge B}(\mathscr{S})$ qui admet un adjoint à droite. On dispose de plusieurs structures de catégories de modèles fermées sur la catégorie $\mathbf{B i s p t}^{A, B}(\mathscr{S})$ (où on peut décréter que les cofibrations sont les monomorphismes). On a tout d'abord une structure dans laquelle les équivalences faibles sont les $I$-équivalences terme à terme. Comme pour les spectres, on a une notion de $\Omega$-bispectre, il y en a même plusieurs :

- bispectres dont les $A$-spectres-lignes sont des $\Omega$-spectres;

- bispectres dont les $B$-spectres-colonnes sont des $\Omega$-spectres;

- bispectres vérifiant simultanément les deux conditions précédentes (ce sont ceux-là que l'on appellera « $\Omega$-bispectres » et donneront naissance à la notion d'équivalence stable de bispectres).

En procédant comme pour les spectres, on obtient ainsi trois nouvelles structures de catégorie de modèles. Soit $\mathcal{S H}^{A, B}(\mathscr{S}, I)$ la catégorie homotopique de $\operatorname{Bispt}^{A, B}(\mathscr{S})$ obtenue en inversant les équivalences stables. En remarquant que l'on dispose d'une adjonction de Quillen, on montre facilement que $\Delta: \operatorname{Bispt}^{A, B}(\mathscr{S}) \rightarrow \mathbf{S p t}^{A \wedge B}(\mathscr{S})$ induit une équivalence de catégories $\mathcal{S H}^{A, B}(\mathscr{S}, I) \stackrel{\sim}{\rightarrow} \mathcal{S H}^{A \wedge B}(\mathscr{S}, I)$.

Comme $\mathcal{S H}{ }^{A \wedge B}(\mathscr{S}, I)$ est équivalente à $\mathcal{S H}^{A, B}(\mathscr{S}, I)$ (de façon compatible avec les foncteurs de suspension), il s'agit de montrer que le foncteur de suspension induit une autoéquivalence de la catégorie $\mathcal{S H}^{A, B}(\mathscr{S}, I)$. La catégorie de modèles $\operatorname{Bispt}^{A, B}(\mathscr{S})$ donnant naissance à la catégorie homotopique $\mathcal{S} \mathcal{H}^{A, B}(\mathscr{S}, I)$ est une localisation à la Bousfield de la structure de catégorie de modèles sur Bispt ${ }^{A, B}(\mathscr{S})$ obtenue en exploitant la notion de bispectre dont les $A$-spectres lignes sont des $\Omega$-spectres (cf. ci-dessus). D'après la proposition 3.8, il suffit de montrer que le foncteur de suspension induit une autoéquivalence de la catégorie homotopique de cette dernière catégorie de modèles, ce qui résulte facilement de notre hypothèse sur $\mathcal{S H}^{A}(\mathscr{S}, I)$.

Démontrons le théorème 3.10. Grâce au corollaire 2.20, on peut supposer que $T=S^{1} \wedge T^{\prime}$ avec $T^{\prime} \in \operatorname{Esp}_{\mathbf{0}}(\mathscr{S})$. Le lemme montre qu'il suffit de traiter le cas $T=S^{1}$. D'après la proposition 3.8 , on peut supposer que $I=\bullet$. Dans le cas où $\mathscr{S}$ est le site ponctuel, le résultat est classique et très facile. Le cas d'un site $\mathscr{S}$ plus général s'y ramène. Dans le cas où le site $\mathscr{S}$ admet suffisamment de foncteurs fibres, cette réduction est triviale. Dans le cas général, le résultat est donc connu pour le site formé par la catégorie sous-jacente au site $\mathscr{S}$ munie

TOME $135-2007-\mathrm{N}^{\circ} 4$ 
de la topologie grossière; en vertu de la proposition 3.8 , le cas du site $\mathscr{S}$ s'en déduit par localisation à la Bousfield ${ }^{(7)}$.

REmARQUe 3.12. - Sous les hypothèses du théorème 3.10, il est évident que les foncteurs $\operatorname{Hom}_{\mathcal{S} \mathcal{H}^{T}(\mathscr{S}, I)}\left(F_{n} X_{+},-\right)$pour $n \in \mathbf{N}$ et $X \in \mathscr{S}$ forment une famille conservative de foncteurs cohomologiques $\mathcal{S H}^{T}(\mathscr{S}, I) \rightarrow \mathbf{A b}$. On peut ainsi généraliser la définition des weighted stable homotopy groups de [20, § 3.2]. On peut passer ces préfaisceaux d'homotopie stable au foncteur faisceau associé pour obtenir des faisceaux d'homotopie stable et vérifier qu'on obtient encore une famille conservative de foncteurs cohomologiques de $\mathcal{S H}^{T}(\mathscr{S}, I)$ vers la catégorie des faisceaux de groupes abéliens sur $\mathscr{S}$.

3.3. $T$-suspensions, $T$-espaces de lacets. - Nous allons étudier ici les foncteurs de $T$-suspension et les foncteurs « $T$-espaces de lacets ». Dans le cas particulier des catégories $\mathcal{S H}(S)$ (cf. section 4), la conclusion de cette étude pourrait contribuer à une démonstration alternative du théorème 3.10.

On se donne un site suspendu avec intervalle $(\mathscr{S}, I, T)$.

DÉfinition 3.13. - Soit $X \in \mathbf{E s p}_{\bullet}(\mathscr{S})$, on note $\Sigma_{X}: \operatorname{Spt}^{T}(\mathscr{S}) \rightarrow \mathbf{S p t}^{T}(\mathscr{S})$ le foncteur qui à un $T$-spectre $\mathbf{E}$ associe le $T$-spectre $\Sigma_{X} \mathbf{E}$ tel que $\left(\Sigma_{X} \mathbf{E}\right)_{n}=$ $\mathbf{E}_{n} \wedge X$ et où les morphismes d'assemblage sont les morphismes évidents.

Le lemme suivant est trivial.

LEMme 3.14. - Le foncteur $\Sigma_{X}: \mathbf{S p t}^{T}(\mathscr{S}) \rightarrow \mathbf{S p t}^{T}(\mathscr{S})$ admet un foncteur adjoint à droite $\Omega_{X}: \mathbf{S p t}^{T}(\mathscr{S}) \rightarrow \mathbf{S p t}^{T}(\mathscr{S})$ tel que si $\mathbf{E}$ est un T-spectre, $\left(\Omega_{X} \mathbf{E}\right)_{n}=$ Hom. $_{\bullet}\left(X, \mathbf{E}_{n}\right)$, les morphismes d'assemblage de $\Omega_{X}$ étant les morphismes évidents.

Proposition 3.15. - Pour tout $X \in \mathbf{E s p}_{\mathbf{0}}(\mathscr{S})$, les foncteurs $\left(\Sigma_{X}, \Omega_{X}\right)$ définissent une adjonction de Quillen pour les (quatre) structures de catégories de modèles définies sur $\mathbf{S p t}^{T}(\mathscr{S})$.

Le point essentiel est de montrer que $\Sigma_{X}$ préserve les équivalences stables, ce qui résulte facilement des définitions, le foncteur dérivé à droite de $\Omega_{X}$ pour la structure projective préservant les $\Omega$-spectres.

DÉFInItion 3.16. - On note $\Sigma_{X}: \mathcal{S H}^{T}(\mathscr{S}, I) \rightarrow \mathcal{S H}^{T}(\mathscr{S}, I)$ le foncteur induit par le foncteur du même nom sur $\operatorname{Spt}^{T}(\mathscr{S})$, son adjoint à droite est $\mathrm{R} \Omega_{X}: \mathcal{S} \mathcal{H}^{T}(\mathscr{S}, I) \rightarrow \mathcal{S H} \mathcal{H}^{T}(\mathscr{S}, I):$ c'est le foncteur dérivé à droite de $\Omega_{X}$ pour la structure stable sur $\operatorname{Spt}^{T}(\mathscr{S})$.

(7) Le théorème [10, theorem 1.1], utilisé à la section 5, montre plus précisément qu'il s'agit de la localisation à la Bousfield par rapport aux morphismes de la forme $F_{n} \mathscr{U}_{+} \rightarrow F_{n} X_{+}$où $\mathscr{U} \rightarrow X$ parcourt les hyper-recouvrements dans $\mathscr{S}$ et $n$ les entiers naturels. 
Du fait de cette adjonction, le foncteur $\Sigma_{X}: \mathcal{S H}^{T}(\mathscr{S}, I) \rightarrow \mathcal{S H}^{T}(\mathscr{S}, I)$ est une équivalence de catégories si et seulement si les morphismes d'adjonction $\operatorname{id}_{\mathcal{S} \mathcal{H}^{T}(\mathscr{S}, I)} \rightarrow \mathrm{R} \Omega_{X} \circ \Sigma_{X}$ et $\Sigma_{X} \circ \mathrm{R} \Omega_{X} \rightarrow \operatorname{id}_{\mathcal{S H} \mathcal{H}^{T}(\mathscr{S}, I)}$ sont des isomorphismes, ce qui équivaut encore à dire que $\mathrm{R} \Omega_{X}: \mathcal{S} \mathcal{H}^{T}(\mathscr{S}, I) \rightarrow \mathcal{S} \mathcal{H}^{T}(\mathscr{S}, I)$ est une équivalence de catégories, auquel cas $\Sigma_{X}$ et $\mathrm{R} \Omega_{X}$ seraient quasi-inverses l'un de l'autre.

Proposition 3.17. - On se donne deux objets $A$ et $B$ de Esp.(S). Les conditions suivantes sont équivalentes :

- le foncteur $\Sigma_{A \wedge B}: \mathcal{S H}^{T}(\mathscr{S}, I) \rightarrow \mathcal{S H}^{T}(\mathscr{S}, I)$ est une équivalence de catégories;

- les deux foncteurs $\Sigma_{A}, \Sigma_{B}: \mathcal{S H}^{T}(\mathscr{S}, I) \rightarrow \mathcal{S H}^{T}(\mathscr{S}, I)$ sont des équivalences de catégories.

Cela résulte simplement du fait que l'on a des isomorphismes (canoniques) de foncteurs $\mathcal{S H}^{T}(\mathscr{S}, I) \rightarrow \mathcal{S H}^{T}(\mathscr{S}, I)$ :

$$
\Sigma_{A} \circ \Sigma_{B} \simeq \Sigma_{B} \circ \Sigma_{A} \simeq \Sigma_{A \wedge B} .
$$

Le point essentiel est que $\Sigma_{A}$ et $\Sigma_{B}$ 《commutent». On peut en effet conclure en utilisant l'argument permettant de dire que dans un monoïde $M$, si deux éléments $f$ et $g$ commutent, alors $f g$ est inversible si et seulement si $f$ et $g$ sont inversibles.

REMARque 3.18. - Grâce à cette proposition, on peut montrer que s'il existe $T^{\prime} \in \mathscr{H}_{\bullet}(\mathscr{S}, I)$ et un isomorphisme $T \simeq S^{1} \wedge T^{\prime}$ dans $\mathscr{H}_{\bullet}(\mathscr{S}, I)$, alors pour montrer que $\mathcal{S H}^{T}(\mathscr{S}, I)$ est triangulée, il suffit de montrer que $\Sigma_{T}: \mathcal{S H}^{T}(\mathscr{S}, I) \rightarrow$ $\mathcal{S H}^{T}(\mathscr{S}, I)$ est une équivalence de catégories.

DÉFinition 3.19. - On note $\Sigma_{T}^{1}: \mathbf{S p t}^{T}(\mathscr{S}) \rightarrow \mathbf{S p t}^{T}(\mathscr{S})$ le foncteur qui à un $T$-spectre $\mathbf{E}$ associe le $T$-spectre $\Sigma_{T}^{1} \mathbf{E}$ dont le $n$-ième terme est $\left(\Sigma_{T}^{\mathrm{l}} \mathbf{E}\right)_{n}=T \wedge \mathbf{E}_{n}$ et dont les morphismes d'assemblage sont définis ainsi :

$$
T \wedge\left(\Sigma_{T}^{1} \mathbf{E}\right)_{n}=T \wedge\left(T \wedge \mathbf{E}_{n}\right) \stackrel{T \wedge \sigma_{n}}{\longrightarrow} T \wedge \mathbf{E}_{n+1}=\left(\Sigma_{T}^{1} E\right)_{n+1}
$$

où $\sigma_{n}: T \wedge \mathbf{E}_{n} \rightarrow \mathbf{E}_{n+1}$ est un morphisme d'assemblage de $\mathbf{E}$.

Je prends ici les mêmes notations que dans [20, remark 2.4]. Cependant, compte tenu de la plus grande généralité envisagée ici, nous donnerons parfois des démonstrations différentes de certains résultats de [20] concernant ces foncteurs; certains résultats intermédaires, comme le lemme 3.26, sont intéressants pour eux-mêmes. Le lemme suivant est évident : 
Lemme 3.20. - Le foncteur $\Sigma_{T}^{l}: \mathbf{S p t}^{T}(\mathscr{S}) \rightarrow \mathbf{S p t}^{T}(\mathscr{S})$ admet un foncteur adjoint à droite $\Omega_{T}^{l}: \mathbf{S p t}^{T}(\mathscr{S}) \rightarrow \mathbf{S p t}^{T}(\mathscr{S})$ tel que pour tout $T$-spectre $\mathbf{E}$, pour tout entier naturel $n$, on ait $\left(\Omega_{T}^{l} \mathbf{E}\right)_{n}=\operatorname{Hom} .\left(T, \mathbf{E}_{n}\right)$ et que le morphisme d'« assemblage » soit défini à partir du morphisme $\tilde{\sigma}_{n}: \mathbf{E}_{n} \rightarrow \operatorname{Hom}_{\bullet}\left(T, \mathbf{E}_{n+1}\right)$ de la manière suivante :

$$
\left(\Omega_{T}^{l} \mathbf{E}\right)_{n}=\operatorname{Hom}_{\bullet}\left(T, \mathbf{E}_{n}\right) \stackrel{\operatorname{Hom}_{\bullet}\left(T, \tilde{\sigma}_{n}\right)}{\longrightarrow} \operatorname{Hom}_{\bullet}\left(T,\left(\Omega_{T}^{l} \mathbf{E}\right)_{n+1}\right) .
$$

Le couple de foncteurs $\left(\Sigma_{T}^{l}, \Omega_{T}^{l}\right)$ définit une adjonction de Quillen pour les quatre structures de catégorie de modèles envisagées sur $\mathbf{S p t}^{T}(\mathscr{S})$.

Remarque 3.21. - Les deux foncteurs $\Omega_{T}$ et $\Omega_{T}^{1} T$ ne sont pas identiques. En effet, si on se hasarde à prendre une notation fonctionnelle et que l'on note $(t, x) \mapsto \sigma(t, x)$ le morphisme d'assemblage $T \wedge \mathbf{E}_{n} \rightarrow \mathbf{E}_{n+1}$, on peut décrire les deux morphismes

$$
\operatorname{Hom}_{\bullet}\left(T, \mathbf{E}_{n}\right) \rightarrow \operatorname{Hom}_{\bullet}\left(T, \operatorname{Hom}_{\bullet}\left(T, \mathbf{E}_{n+1}\right)\right)
$$

définissant les morphismes d'assemblage sur les $T$-spectres $\Omega_{X} \mathbf{E}$ et $\Omega_{T}^{1} \mathbf{E}$. Pour $\Omega_{X} \mathbf{E}$, on obtient :

$$
f \longmapsto t \mapsto t^{\prime} \mapsto \sigma\left(t, f\left(t^{\prime}\right)\right),
$$

tandis que pour $\Omega_{T}^{1} \mathbf{E}$, on trouve :

$$
f \longmapsto t \mapsto t^{\prime} \mapsto \sigma\left(t^{\prime}, f(t)\right) .
$$

THÉorìme 3.22. - Le foncteur $\Sigma_{T}^{l}: \mathbf{S p t}^{T}(\mathscr{S}) \rightarrow \mathbf{S p t}^{T}(\mathscr{S})$ induit une équivalence de catégories $\Sigma_{T}^{l}: \mathcal{S H}^{T}(\mathscr{S}, I) \rightarrow \mathcal{S H}^{T}(\mathscr{S}, I)$.

La démonstration de cette proposition utilise d'autres constructions, utiles par ailleurs, cf. [16] :

DÉfinition 3.23. - On note $s_{-}: \mathbf{S p t}^{T}(\mathscr{S}) \rightarrow \mathbf{S p t}^{T}(\mathscr{S})$ le foncteur tel que pour tout $T$-spectre $\mathbf{E}, s_{-} \mathbf{E}=\left(\mathbf{E}_{1}, \mathbf{E}_{2}, \ldots\right)$ et $s_{+}: \mathbf{S p t}^{T}(\mathscr{S}) \rightarrow \mathbf{S p t}^{T}(\mathscr{S})$ le foncteur tel que l'on ait $s_{+} \mathbf{E}=\left(\bullet, \mathbf{E}_{0}, \mathbf{E}_{1}, \ldots\right)$, les morphismes d'assemblage sur $s_{-} \mathbf{E}$ et $s_{+} \mathbf{E}$ étant définis de façon évidente.

On montre aisément que $s_{-}$est l'adjoint à droite de $s_{+}$. Comme $s_{-}$préserve les $\Omega$-spectres, on obtient que $s_{+}$préserve les équivalences stables et que $\left(s_{+}, s_{-}\right)$forme une adjonction de Quillen pour les quatre structures de catégories de modèles définies sur $\mathbf{S p t}^{T}(\mathscr{S})$. Le lemme suivant montre qu'il n'est pas nécessaire de dériver à droite $s_{-}$pour la structure stable :

Lemme 3.24. - Le foncteur $s_{-}: \mathbf{S p t}^{T}(\mathscr{S}) \rightarrow \mathbf{S p t}^{T}(\mathscr{S})$ préserve les équivalences stables. 
On note $s_{\mathrm{d}}: \mathbf{S p t}^{T}(\mathscr{S}) \rightarrow \mathbf{S p t}^{T}(\mathscr{S})$ le foncteur qui à un $T$-spectre $\mathbf{E}$ associe le $T$-spectre $\left(\mathbf{H o m}_{\bullet}\left(T, \mathbf{E}_{0}\right), \mathbf{E}_{0}, \mathbf{E}_{1}, \ldots\right)$, les morphismes d'assemblage étant définis de façon évidente. Il est clair que $s_{\mathrm{d}}$ est l'adjoint à droite de $s_{-}$. On remarque que si $\mathbf{E}$ est un $\Omega$-spectre injectivement fibrant, alors $s_{\mathrm{d}} \mathbf{E}$ est itou. On en déduit que $s_{-}$préserve les équivalences stables.

Lemme 3.25. - Les foncteurs adjoints $\left(s_{+}, s_{-}\right)$induisent des autoéquivalences de $\mathcal{S H}^{T}(\mathscr{S}, I)$ inverses l'une de l'autre.

Les foncteurs $s_{-}$et $s_{+} \operatorname{sur} \mathbf{S p t}^{T}(\mathscr{S})$ préservent tous les deux les équivalences stables. Il s'agit donc de montrer que pour tout $T$-spectre $\mathbf{E}$, les morphismes d'adjonction $\mathbf{E} \rightarrow s_{-} s_{+} \mathbf{E}$ et $s_{+} s_{-} \mathbf{E} \rightarrow \mathbf{E}$ sont des équivalences stables. Pour le premier morphisme, c'est évident puisqu'il s'agit d'un isomorphisme. Pour le second, c'est l'objet du lemme suivant :

Lemme 3.26. - Pour tout objet $\mathbf{E}$ de $\mathbf{S p t}^{T}(\mathscr{S})$, le morphisme canonique $s_{+} s_{-} \mathbf{E} \rightarrow \mathbf{E}$ est une équivalence stable.

On considère le morphisme canonique $F_{0} \mathbf{E}_{0} \rightarrow \mathbf{E}$ et le diagramme commutatif auquel il donne naissance :

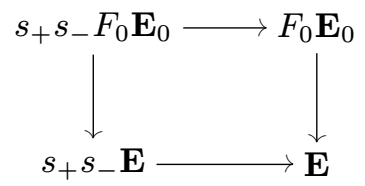

On observe que $s_{+} s_{-} F_{0} \mathbf{E}_{0}=F_{1}\left(T \wedge \mathbf{E}_{0}\right)$. On peut alors vérifier que ce carré est cocartésien. De plus, le morphisme d'en haut $F_{1}\left(T \wedge \mathbf{E}_{0}\right) \rightarrow F_{0} \mathbf{E}_{0}$ est un monomorphisme et une équivalence stable (cf. remarque 1.23). On peut donc conclure puisque la structure de catégorie de modèles du théorème 1.21 est propre à gauche.

Le lemme suivant nous ramène à ce qui nous préoccupait :

LEMme 3.27. - Il existe un morphisme canonique de foncteurs

$$
\varsigma: \operatorname{id}_{\mathbf{S p t}^{T}(\mathscr{S})} \rightarrow \Omega_{T}^{l} \circ s_{-}: \mathbf{S p t}^{T}(\mathscr{S}) \rightarrow \mathbf{S p t}^{T}(\mathscr{S}) .
$$

Pour tout $T$-spectre $\mathbf{E}$, on définit $\varsigma: \mathbf{E}_{n} \rightarrow\left(\Omega_{T}^{1} s_{-} \mathbf{E}\right)_{n}=\operatorname{Hom}_{\bullet}\left(T, \mathbf{E}_{n+1}\right)$ de manière évidente : c'est le morphisme d'assemblage de $\mathbf{E}$. Le point délicat consiste à vérifier que $\varsigma$ est bien un morphisme de $T$-spectres (ce qui serait faux si on remplaçait $\Omega_{T}^{1}$ par $\Omega_{T}$, subtilité fort contrariante).

$\mathrm{Au}$ niveau des foncteurs dérivés pour la structure stable $\operatorname{sur} \mathbf{S p t}^{T}(\mathscr{S})$, le morphisme de foncteurs donné par ce lemme est un isomorphisme (considérer le cas où $\mathbf{E}$ est un $\Omega$-spectre injectivement fibrant). Comme on a montré que $s_{-}: \mathcal{S H}^{T}(\mathscr{S}, I) \rightarrow \mathcal{S H}^{T}(\mathscr{S}, I)$ est une autoéquivalence de catégories, il 
découle du lemme que $\mathrm{R} \Omega_{T}^{\mathrm{l}}$ induit aussi une autoéquivalence de $\mathcal{S} \mathcal{H}^{T}(\mathscr{S}, I)$. Par adjonction, l'adjoint à gauche $\Sigma_{T}^{\mathrm{l}}: \mathcal{S} \mathcal{H}^{T}(\mathscr{S}, I) \rightarrow \mathcal{S} \mathcal{H}^{T}(\mathscr{S}, I)$ de $\mathrm{R} \Omega_{T}^{\mathrm{l}}$ est également une équivalence de catégories, ce qui achève la démonstration du théorème 3.22 .

Remarque 3.28. - Si on dispose d'une homotopie explicite entre l'identité et la permutation circulaire sur $T \wedge T \wedge T$, alors [20, lemma 3.20] (dont la démonstration vaut encore dans ce cadre) permet d'obtenir un isomorphisme de foncteurs $\Sigma_{T} \cong \Sigma_{T}^{l}: \mathcal{S} \mathcal{H}^{T}(\mathscr{S}, I) \rightarrow \mathcal{S} \mathcal{H}^{T}(\mathscr{S}, I)$; comme on sait que $\Sigma_{T}^{\mathrm{l}}$ est une autoéquivalence, il en va de même pour $\Sigma_{T}$, ce qui permet de retrouver que $\mathcal{S H}^{T}(\mathscr{S}, I)$ est une catégorie triangulée (cf. remarque 3.18).

3.4. Espaces de $T$-lacets infinis. - Cette sous-section va permettre de faire le lien entre la construction de $\mathcal{S} \mathcal{H}^{T}(\mathscr{S}, I)$ donnée ici, et l'approche utilisée dans [20].

On se donne toujours un site suspendu avec intervalle $(\mathscr{S}, I, T)$.

DÉFInition 3.29. - On note $\boldsymbol{\Lambda}: \mathbf{S p t}^{T}(\mathscr{S}) \rightarrow \mathbf{S p t}^{T}(\mathscr{S})$ le foncteur $\Omega_{T}^{\mathrm{l}} \circ s_{-}$ et $\varsigma: \operatorname{id}_{\mathbf{S p t}^{T}(\mathscr{S})} \rightarrow \boldsymbol{\Lambda}$ la transformation naturelle canonique (cf. lemme 3.27).

On peut faire l'observation cruciale suivante (cf. [16, lemma 4.5]) :

Lemme 3.30. - Pour tout objet $\mathbf{E}$ de $\mathbf{S p t}^{T}(\mathscr{S})$, on a l'égalité de morphismes

$$
\boldsymbol{\Lambda}\left(\varsigma_{\mathbf{E}}\right)=\varsigma_{\boldsymbol{\Lambda} \mathbf{E}}: \mathbf{\Lambda} \mathbf{E} \rightarrow \boldsymbol{\Lambda}^{2} \mathbf{E} .
$$

On peut donc définir un système inductif

$$
\mathbf{E} \rightarrow \mathbf{\Lambda} \mathbf{E} \rightarrow \mathbf{\Lambda}^{2} \mathbf{E} \rightarrow \mathbf{\Lambda}^{3} \mathbf{E} \rightarrow \ldots
$$

pour tout $T$-spectre $\mathbf{E}$ sans qu'il y ait ambiguité sur la définition des flèches de transition.

DÉfinition 3.31. - Pour tout objet $\mathbf{E}$ de $\mathbf{S p t}^{T}(\mathscr{S})$, on note $\boldsymbol{\Lambda}^{\infty} \mathbf{E}$ la colimite du système inductif ci-dessus.

Lemme 3.32. - Pour tout objet $\mathbf{E}$ dans $\mathbf{S p t}^{T}(\mathscr{S})$, le morphisme canonique

$$
\boldsymbol{\Lambda}^{\infty}\left(\varsigma_{\mathbf{E}}\right): \boldsymbol{\Lambda}^{\infty}(\mathbf{E}) \rightarrow \boldsymbol{\Lambda}^{\infty}(\boldsymbol{\Lambda} \mathbf{E})
$$

est un isomorphisme. Si le foncteur $\operatorname{Hom}_{\bullet}(T,-)$ sur $\mathbf{E s p}_{\bullet}(\mathscr{S})$ commute aux colimites indexées par $\mathbf{N}$, alors la flèche

$$
\varsigma_{\Lambda^{\infty} \mathbf{E}}: \boldsymbol{\Lambda}^{\infty} \mathbf{E} \rightarrow \boldsymbol{\Lambda}\left(\boldsymbol{\Lambda}^{\infty} \mathbf{E}\right)
$$

est un isomorphisme.

C'est évident à partir du lemme 3.30 . 
DÉFinition 3.33 (propriété $(J))$. - On dit que $(\mathscr{S}, I, T)$ satisfait la propriété $(\mathrm{J})$ si les deux conditions suivantes sont vérifiées :

$(\mathrm{J} 1)$ Le foncteur $\mathbf{H o m}_{\bullet}(T,-): \mathbf{E s p}_{\bullet}(\mathscr{S}) \rightarrow \mathbf{E s p}_{\bullet}(\mathscr{S})$ commute aux colimites indexées par $\mathbf{N}$;

(J2) Pour tout système inductif

$$
X_{0} \rightarrow X_{1} \rightarrow X_{2} \rightarrow \ldots
$$

d'objets $I$-fibrants de Esp. $(\mathscr{S})$ indexé par $\mathbf{N}$, si on note $X_{\infty}$ la colimite de ce système, alors $X_{\infty}$ est acyclique pour le foncteur $\operatorname{Hom}_{\bullet}(T,-)$, autrement dit si $X_{\infty} \rightarrow X_{\infty}^{\prime}$ est une $I$-équivalence faible avec $X_{\infty}^{\prime} I$ fibrant dans $\operatorname{Esp}_{\bullet}(\mathscr{S})$, alors

$$
\operatorname{Hom}_{\bullet}\left(T, X_{\infty}\right) \rightarrow \operatorname{Hom}_{\bullet}\left(T, X_{\infty}^{\prime}\right)
$$

est une $I$-équivalence faible.

Il est immédiat que les foncteurs $\boldsymbol{\Lambda}$ et $\boldsymbol{\Lambda}^{\infty}$ préservent les $I$-équivalences faibles projectives entre objets projectivement $I$-fibrants ; mais a priori si $\mathbf{E}$ est projectivement $I$-fibrant, $\boldsymbol{\Lambda}^{\infty}$ ne l'est pas forcément, c'est ce qui justifie l'introduction de la condition (J2) ci-dessus. On note $\mathrm{R} \Lambda: \mathcal{S H}_{\mathrm{p}}^{T}(\mathscr{S}, I) \rightarrow \mathcal{S H}_{\mathrm{p}}^{T}(\mathscr{S}, I)$ $\left(\right.$ resp. $\left.\mathrm{R} \boldsymbol{\Lambda}^{\infty}: \mathcal{S} \mathcal{H}_{\mathrm{p}}^{T}(\mathscr{S}, I) \rightarrow \mathcal{S} \mathcal{H}_{\mathrm{p}}^{T}(\mathscr{S}, I)\right)$ les foncteurs dérivés de $\boldsymbol{\Lambda}$ et $\boldsymbol{\Lambda}^{\infty}$ pour la structure projective. On dispose de transformations naturelles évidentes $\varsigma: \operatorname{id}_{\mathcal{S} \mathcal{H}_{\mathrm{p}}^{T}(\mathscr{S}, I)} \rightarrow \mathrm{R} \boldsymbol{\Lambda}$ et $\varsigma^{\infty}: \operatorname{id}_{\mathcal{S H}} \mathcal{H}_{\mathrm{p}}^{T}(\mathscr{S}, I) \rightarrow \mathrm{R} \boldsymbol{\Lambda}^{\infty}$.

LEMME 3.34. - Soit $\mathbf{E} \in \mathcal{S H}_{\mathrm{p}}^{T}(\mathscr{S}, I)$. Alors, $\mathbf{E} \in \mathcal{S H}_{\Omega}^{T}(\mathscr{S}, I)$ si et seulement si $\varsigma_{\mathbf{E}}: \mathbf{E} \rightarrow \mathrm{R} \boldsymbol{\Lambda}(\mathbf{E})$ est un isomorphisme dans $\mathcal{S H}_{\mathrm{p}}^{T}(\mathscr{S}, I)$.

C'est évident.

ThÉORÈme 3.35. - Soit $(\mathscr{S}, I, T)$ un site suspendu avec intervalle satisfaisant la propriété $(J)$. Alors,

(1) L'image de $\mathrm{R} \boldsymbol{\Lambda}^{\infty}: \mathcal{S H}_{\mathrm{p}}^{T}(\mathscr{S}, I) \rightarrow \mathcal{S H}_{\mathrm{p}}^{T}(\mathscr{S}, I)$ est contenue dans $\mathcal{S H}_{\Omega}^{T}(\mathscr{S}, I) ;$

(2) Pour tout $\mathbf{E}$ in $\mathcal{S H}_{\mathrm{p}}^{T}(\mathscr{S}, I)$, on a l'égalité de morphismes $\mathrm{R} \mathbf{\Lambda}^{\infty} \mathbf{E} \rightarrow$ $\mathrm{R} \boldsymbol{\Lambda}^{\infty}\left(\mathrm{R} \boldsymbol{\Lambda}^{\infty} \mathbf{E}\right)$ :

$$
\mathrm{R} \boldsymbol{\Lambda}^{\infty}\left(\varsigma_{\mathbf{E}}^{\infty}\right)=\varsigma_{\mathrm{R} \mathbf{\Lambda}^{\infty} \mathbf{E}}^{\infty}
$$

(3) Le foncteur $\mathrm{R} \boldsymbol{\Lambda}^{\infty}: \mathcal{S H}_{\mathrm{p}}^{T}(\mathscr{S}, I) \rightarrow \mathcal{S} \mathcal{H}_{\Omega}^{T}(\mathscr{S}, I)$ est adjoint à gauche de l'inclusion $\mathcal{S H}_{\Omega}^{T}(\mathscr{S}, I) \rightarrow \mathcal{S H}_{\mathrm{p}}^{T}(\mathscr{S}, I)$;

(4) Un morphisme $f: \mathbf{E} \rightarrow \mathbf{F}$ dans $\mathcal{S H}_{\mathrm{p}}^{T}(\mathscr{S}, I)$ est une I-équivalence stable si et seulement si le morphisme $\mathrm{R} \boldsymbol{\Lambda}^{\infty}(f): \mathrm{R} \boldsymbol{\Lambda}^{\infty}(\mathbf{E}) \rightarrow \mathrm{R} \boldsymbol{\Lambda}^{\infty}(\mathbf{F})$ est un isomorphisme dans $\mathcal{S H}_{\mathrm{p}}^{T}(\mathscr{S}, I)$; 
(5) Pour tout objet $\mathbf{E} \in \mathcal{S} \mathcal{H}_{\mathrm{p}}^{T}(\mathscr{S}, I)$, les morphismes

$$
\varsigma: \mathbf{E} \rightarrow \mathrm{R} \boldsymbol{\Lambda}(\mathbf{E}), \quad \varsigma^{\infty}: \mathbf{E} \rightarrow \mathrm{R} \boldsymbol{\Lambda}^{\infty}(\mathbf{E})
$$

sont des I-équivalences stables.

La démonstration est purement soritale.

Remarque 3.36. - D'après [20, § 2.2], les hypothèse du théorème 3.35 sont vérifiées pour le site suspendu avec intervalle $\left(\mathrm{Sm} / S_{\mathrm{Nis}}, \mathbf{A}^{1}, \mathbf{P}^{1}\right)$ considéré à la section 4. Grâce à la conclusion (4) du théorème, on obtient que les notions d'équivalences stables définies ici et dans [20] sont les mêmes.

REMARQUe 3.37. - Sous les hypothèses du théorème 3.35, on obtient que pour tout $T$-spectre $\mathbf{E}$ projectivement fibrant, la flèche $\varsigma: \mathbf{E} \rightarrow \mathbf{\Lambda} \mathbf{E}$ qui a été introduite au lemme 3.27 est une $I$-équivalence stable. Je ne sais malheureusement pas établir ce résultat autrement qu'en utilisant la propriété $(\mathrm{J})$. Pour cette raison, j'ignore si dans le cas général, il existe un analogue de [24, lemma 3.20, page 93] obtenu en itérant $\boldsymbol{\Lambda}$ (et des résolutions projectives) à une puissance donnée par un «gros » ordinal.

\section{Catégories homotopiques stables d'un schéma noethérien}

DÉfinition 4.1. - Soit $S$ un schéma noethérien. On note $\mathrm{Sm} / S_{\mathrm{Nis}}$ le site formé par la catégorie $\mathrm{Sm} / S$ des schémas lisses, séparés et de type fini sur $S$, munie de la topologie de Nisnevich (aussi appelée « hensélienne », cf. [21]). On pose $\mathcal{S H}(S)=\mathcal{S} \mathcal{H}^{\mathbf{P}^{1}}\left(\mathrm{Sm} / S_{\mathrm{Nis}}, \mathbf{A}^{1}\right)$, où $\mathbf{P}^{1}$ est pointé par $\infty$. On note aussi $\mathscr{H}(S)$ et $\mathscr{H}_{\bullet}(S)$ les catégories homotopiques associées au site avec intervalle $\left(\mathrm{Sm} / S_{\mathrm{Nis}}, \mathbf{A}^{1}\right)$.

D'après la remarque 3.36, la catégorie $\mathcal{S H}(S)$ définie ici est équivalente à celle de [20]. Comme on dispose d'un isomorphisme $\left(\mathbf{P}^{1}, \infty\right) \simeq S^{1} \wedge\left(\mathbf{G}_{\mathrm{m}}, 1\right)$ dans $\mathscr{H}_{\bullet}(S)$ (cf. [24, lemma 2.15, page 111] et [24, corollary 2.18, page 112]), le théorème 3.10 implique que $\mathcal{S H}(S)$ est naturellement munie d'une structure catégorie triangulée.

REMARQUE 4.2. - La construction donnée dans cet article permet de définir une version étale de $\mathcal{S H}(S)$ : pour tout schéma noethérien $S$, on pose $\mathcal{S} \mathcal{H}_{\text {ét }}(S)=\mathcal{S H}^{\mathbf{P}^{1}}\left(\mathrm{Sm} / S_{\text {ét }}, \mathbf{A}^{1}\right)$. Le morphisme de sites $\alpha: \mathrm{Sm} / S_{\text {ét }} \rightarrow \mathrm{Sm} / S_{\mathrm{Nis}}$ induit des foncteurs adjoints triangulés $\mathrm{R} \alpha_{\star}: \mathcal{S} \mathcal{H}_{\text {ét }}(S) \rightarrow \mathcal{S H}(S)$ et $\mathrm{L} \alpha^{\star}: \mathcal{S H}(S) \rightarrow \mathcal{S H} \mathcal{H}_{\text {ét }}(S)$. Le foncteur $\mathrm{R} \alpha_{\star}$ permet d'identifier $\mathcal{S} \mathcal{H}_{\text {ét }}(S)$ à une sous-catégorie triangulée de $\mathcal{S H}(S)$. 
Le théorème suivant ne fait que formuler sous forme de triangles distingués les résultats [24, lemma 1.6, page 98], [24, theorem 2.23, page 115] et [24, proposition 2.29 , page 118 ] :

THÉORÈme 4.3. - Soit $S$ un schéma noethérien.

- Soit $X \in \mathrm{Sm} / S$. Soit $U$ un ouvert de $X$ et $p: V \rightarrow X$ un morphisme étale induisant un isomorphisme au-dessus du fermé $X-U$. On a un triangle distingué de Mayer-Vietoris :

$$
p^{-1}(U)_{+} \rightarrow U_{+} \oplus V_{+} \rightarrow X_{+} \rightarrow p^{-1}(U)_{+}[1]
$$

- Soit $i: Z \rightarrow X$ une immersion fermée dans $\mathrm{Sm} / S$. On note $p: X_{Z} \rightarrow X$ l'éclaté de $Z$ dans $X$ et $U$ l'ouvert complémentaire de $Z$ dans $X$. On note $\nu_{i}$ le fibré normal de l'immersion $i$ et Th $\nu_{i}$ l'espace de Thom de ce fibré vectoriel $^{(8)}$. On a des triangles distingués canoniques :

$$
\begin{gathered}
U_{+} \rightarrow X_{+} \rightarrow \mathrm{Th} \nu_{i} \rightarrow U_{+}[1] \text { et } \\
p^{-1}(Z)_{+} \rightarrow X_{Z_{+}} \oplus Z_{+} \rightarrow X_{+} \rightarrow p^{-1}(Z)_{+}[1] .
\end{gathered}
$$

Proposition 4.4. - Pour tout morphisme $f: X \rightarrow S$ entre schémas noethériens, on dispose de foncteurs adjoints triangulés $\mathrm{L} f^{\star}: \mathcal{S H}(S) \rightarrow \mathcal{S H}(X)$ et $\mathrm{R} f_{\star}: \mathcal{S H}(X) \rightarrow \mathcal{S H}(S)$;

- soit $S$ un schéma noethérien, soit $X \in \mathrm{Sm} / S$, on note $f: X \rightarrow S$ le morphisme structural (lisse), alors le foncteur $\mathrm{L} f^{\star}: \mathcal{S H}(S) \rightarrow \mathcal{S H}(X)$ admet un adjoint à gauche triangulé $\mathrm{L} f_{\sharp}: \mathcal{S H}(X) \rightarrow \mathcal{S H}(S)$.

Si $f: X \rightarrow S$ est un morphisme entre schémas noethériens, une application continue de sites avec intervalle $\left(\mathrm{Sm} / X_{\mathrm{Nis}}, \mathbf{A}^{1}\right) \rightarrow\left(\mathrm{Sm} / S_{\mathrm{Nis}}, \mathbf{A}^{1}\right)$ a été définie dans [24, page 108]. Notons $\left(f^{\star}, f_{\star}\right)$ les foncteurs correspondant au niveau des espaces (pointés). Pour pouvoir appliquer le théorème 2.11 qui donnera naissance au couple de foncteurs adjoints (triangulés) $\left(\mathrm{L} f^{\star}, \mathrm{R} f_{\star}\right)$ voulu, il s'agit de définir une application raisonnable de sites suspendus avec intervalles; soit $\mathscr{F} \in \mathbf{E s p}_{\bullet}\left(\mathrm{Sm} / S_{\mathrm{Nis}}\right)$, on définit un isomorphisme canonique :

$$
\Psi_{\mathscr{F}}: f^{\star}\left(\mathbf{P}_{S}^{1} \wedge \mathscr{F}\right) \stackrel{\sim}{\rightarrow} f^{\star}\left(\mathbf{P}_{S}^{1}\right) \wedge f^{\star} \mathscr{F}=\mathbf{P}_{X}^{1} \wedge f^{\star} \mathscr{F}
$$

dans Esp. $\left(\mathrm{Sm} / X_{\mathrm{Nis}}\right)$. Il n'est pas difficile de vérifier que l'on obtient bien ainsi une application raisonnable de sites suspendus avec intervalles $\left(\mathrm{Sm} / X_{\mathrm{Nis}}, \mathbf{A}^{1}, \mathbf{P}^{1}\right) \rightarrow\left(\mathrm{Sm} / S_{\mathrm{Nis}}, \mathbf{A}^{1}, \mathbf{P}^{1}\right)$, cf. définition 2.9.

Supposons $X \in \mathrm{Sm} / S$. Le foncteur $f^{\star}: \operatorname{Prefais}(\mathrm{Sm} / S) \rightarrow \operatorname{Prefais}(\mathrm{Sm} / X)$ est le foncteur «image directe » pour l'application continue $\mathrm{Sm} / S_{\mathrm{Nis}} \rightarrow$ $\mathrm{Sm} / X_{\mathrm{Nis}}$ de sites donnée par le foncteur $\mathrm{Sm} / X \rightarrow \mathrm{Sm} / S$ qui à $Y \stackrel{p}{\rightarrow} X \in \mathrm{Sm} / X$

(8) On rappelle que si $E$ est un fibré vectoriel sur $X \in \mathrm{Sm} / S$, alors Th $E=E /(E-s X)$ où $s: X \rightarrow E$ est la section nulle.

TOME $135-2007-\mathrm{N}^{\circ} 4$ 
associe la composée $Y \stackrel{p}{\rightarrow} X \stackrel{f}{\rightarrow} S \in \mathrm{Sm} / S$. Il est établi dans [24, proposition 2.9, page 108] que l'on définit ainsi une application raisonnable de sites avec intervalles. Notons $f_{\sharp}$ : Esp. $(\mathrm{Sm} / X)_{\mathrm{Nis}} \rightarrow \mathbf{E s p} .(\mathrm{Sm} / S)_{\mathrm{Nis}}$ la version pointée du foncteur «image inverse » associé. La formule de projection [24, proposition 1.23, page 104] implique que l'on a un isomorphisme canonique, pour tout $\mathscr{F} \in \operatorname{Esp}_{\bullet}(\mathrm{Sm} / X)_{\mathrm{Nis}}$ :

$$
\Psi_{\mathscr{F}}: f_{\sharp}\left(\mathscr{F} \wedge \mathbf{P}_{X}^{1}\right) \stackrel{\sim}{\rightarrow} \mathbf{P}_{S}^{1} \wedge f_{\sharp} \mathscr{F} .
$$

On obtient ainsi une application raisonnable de sites suspendus avec intervalles

$$
\left(\mathrm{Sm} / S_{\mathrm{Nis}}, \mathbf{A}^{1}, \mathbf{P}^{1}\right) \rightarrow\left(\mathrm{Sm} / X_{\mathrm{Nis}}, \mathbf{A}^{1}, \mathbf{P}^{1}\right)
$$

donnant un adjoint à gauche $\mathrm{L} f_{\sharp}: \mathcal{S H}(X) \rightarrow \mathcal{S H}(S)$ à $f^{\star}: \mathcal{S H}(S) \rightarrow \mathcal{S H}(X)$.

Proposition 4.5. - Soit $X$ un schéma noethérien, soit $i: Z \rightarrow X$ une immersion fermée et $j: U \rightarrow X$ l'immersion ouverte complémentaire. Alors, il existe un triangle distingué

$$
\mathrm{L} j_{\sharp} j^{\star} \mathbf{E} \rightarrow \mathbf{E} \rightarrow i_{\star} \mathrm{L} i^{\star} \mathbf{E} \rightarrow \mathrm{L} j_{\sharp} j^{\star} \mathbf{E}[1]
$$

fonctoriel en $\mathbf{E}$ dans $\mathcal{S H}(X)$. De plus, pour tout objet $\mathbf{E}$ de $\mathcal{S H}(Z)$, le morphisme d'adjonction $\mathrm{L} i^{\star} i_{\star} \mathbf{E} \rightarrow \mathbf{E}$ est un isomorphisme.

La première chose à remarquer est que le foncteur $i_{\star}: \mathbf{S p t}^{\mathbf{P}^{1}}\left(\mathrm{Sm} / Z_{\mathrm{Nis}}\right) \rightarrow$ $\mathbf{S p t}^{\mathbf{P}^{1}}\left(\mathrm{Sm} / Z_{\mathrm{Nis}}\right)$ préserve les $\mathbf{A}^{1}$-équivalences stables; ce n'est pas tautologique : compte tenu de [24, proposition 2.12, page 109] et du théorème 3.35 , cela résulte de l'isomorphisme canonique $i_{\star} \Lambda \cong \Lambda i_{\star}$.

Pour établir la première assertion de la proposition, la démonstration qui suit consiste à ramener l'énoncé au cas instable (cf. [24, theorem 2.21, page 114]). Soit $\mathbf{E}$ un objet de $\mathbf{S p t}^{\mathbf{P}^{1}}\left(\mathrm{Sm} / X_{\mathrm{Nis}}\right)$. On peut supposer que $\mathbf{E}$ est une résolution admissible $\mathbf{F}_{\text {adm }}$ construite au lemme 2.6. On observe alors que $\mathbf{E}$ est $j^{\star}$-admissible et $i^{\star}$-admissible et même que $j^{\star} \mathbf{E}$ est $j_{\sharp}$-admissible. Pour obtenir le triangle distingué voulu, il suffit donc de montrer que le carré commutatif évident

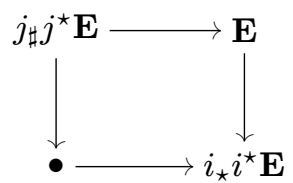

est homotopiquement cocartésien. Il suffit pour cela de vérifier que terme à terme, on obtient un carré homotopiquement cartésien dans Esp. $(\mathrm{Sm} / X)$ (pour la structure de catégorie de modèles donnant naissance à la catégorie $\left.\mathscr{H}_{\bullet}(X)\right)$. La construction de la résolution du lemme 2.6 assure que pour tout entier naturel $n$, l'objet $\mathbf{E}_{n}$ est $j^{\star}$-admissible et $i^{\star}$-admissible et que $j^{\star} \mathbf{E}_{n}$ est 
$j_{\sharp}$-admissible; on s'est bien ramené à la version instable [24, theorem 2.21, page 114].

De même, pour établir la dernière assertion de la proposition, il suffit de montrer que pour tout $A \in \mathscr{H}(Z)$, le morphisme d'adjonction $\mathrm{L} i i^{\star} i_{\star} A \rightarrow A$ est un isomorphisme dans $\mathscr{H}(Z)$. Cet énoncé ne semblant pas figurer dans [24], établissons-le. Tout d'abord, si $A$ est isomorphe à un objet de la forme $\mathrm{L} i{ }^{\star} B$ avec $B \in \mathscr{H}(X)$, c'est vrai puisque le morphisme composé évident

$$
\mathrm{L} i^{\star} B \rightarrow \mathrm{L} i^{\star} i_{\star} \mathrm{L} i^{\star} B \rightarrow \mathrm{L} i^{\star} B
$$

est l'identité et que le morphisme de gauche est un isomorphisme dans $\mathscr{H}(Z)$ (c'est une conséquence du théorème de recollement [24, theorem 2.21, page 114] puisque $\mathrm{L} i{ }^{\star}$ « commute» aux colimites homotopiques, cf. remarque 2.13). Par ailleurs, la sous-catégorie pleine de de $\mathscr{H}(Z)$ formée des $A$ tels que $\mathrm{L} i^{\star} i_{\star} A \rightarrow A$ soit un isomorphisme est stable par «colimite homotopique ». D'après EGA IV 18.1.1, tout schéma lisse sur $Z$ est recouvert par des ouverts de la forme $\pi^{-1}(Z)$ où $\pi: W \rightarrow X$ est un morphisme lisse. D'après le lemme de résolution [24, lemma 1.16, page 52], on peut supposer que $A$ est tel que pour tout entier naturel $n$, le faisceau d'ensembles $A_{n}$ soit somme directe de faisceaux représentés par des schémas de la forme $\pi^{-1}(Z)$ où $\pi: W \rightarrow X$ appartient à $\mathrm{Sm} / X$. Les objets $A_{n}$ étant de la forme $\mathrm{L} i^{\star} B$, la conclusion est connue pour eux. Comme $A \simeq \operatorname{hocolim}_{\Delta \text { opp }} A_{n}$, on a bien $\operatorname{Li}^{\star} i_{\star} A \stackrel{\sim}{\rightarrow} A$.

REMARQUE 4.6. - La proposition 4.5 permet de vérifier l'axiome de localité dans le formalisme des quatre foncteurs (cf. [4]) pour la catégorie bi-fibrée $X \longmapsto \mathcal{S H}(X)$ (pour $X$ parcourant les schémas quasi-projectifs sur une base noethérienne fixée). La fonctorialité construite ici $\left(\mathrm{R} f_{\star}, \mathrm{L} f^{\star}\right.$ pour $f$ quelconque, et $\mathrm{L} f_{\sharp}$ pour $f$ lisse) implique trivialement les autres axiomes des foncteurs homotopiques stables, à l'exception de l'axiome de stabilité qui est pour sa part établi dans [20, §3.4]. Ces axiomes ont été vérifiés de manière indépendante (et différente) par Oliver Röndings. Le travail [4] donne donc des foncteurs $f^{!}: \mathcal{S H}(Y) \rightarrow \mathcal{S H}(X)$ et $f_{!}: \mathcal{S H}(X) \rightarrow \mathcal{S H}(Y)$ satisfaisant de bonnes propriétés pour tout morphisme quasi-projectif $f: X \rightarrow Y$ entre schémas noethériens. Il n'en sera pas fait usage ici.

Remarque 4.7. - Dans la démonstration de la proposition 4.5, on a utilisé que $i_{\star}$ préservait les $\mathbf{A}^{1}$-équivalences stables. J'ignore si ce résultat est encore vrai dans le cas de la topologie étale.

\section{Foncteur « points complexes»}

Le but de cette section est de définir un foncteur triangulé $\mathcal{S H}(\mathbf{C}) \rightarrow \mathcal{S} \mathcal{H}^{\text {top }}$ (et donc plus généralement un foncteur $\mathcal{S H}(S) \rightarrow \mathcal{S} \mathcal{H}^{\text {top }}$ pour tout point 
complexe $\iota:$ Spec $\mathbf{C} \rightarrow S$ de $S$ ), où $\mathcal{S} \mathcal{H}^{\text {top }}$ désigne la catégorie homotopique stable usuelle (c'est-à-dire que $\mathcal{S} \mathcal{H}^{\text {top }}=\mathcal{S} \mathcal{H}^{S^{1}}(\bullet)$ ).

\subsection{Rappels sur les résultats de Dugger, Hollander et Isaksen et conséquences. -} Le théorème suivant est un corollaire de [10, theorem 1.1] :

ThÉORÈme 5.1. - Soit $\mathscr{S}$ un site, soit $\mathscr{F} \in \boldsymbol{\Delta}^{\mathrm{opp}} \operatorname{Prefais}(\mathscr{S})$. Les conditions suivantes sont équivalentes :

(1) Pour tout $X \in \mathscr{S}$, la flèche évidente

$$
\mathscr{F}(X) \rightarrow \mathrm{R} \Gamma(X ; \mathscr{F})
$$

est un isomorphisme dans $\mathscr{H}^{\text {top }}\left(\right.$ où $\mathrm{R} \Gamma(X ;-): \mathscr{H}_{\mathrm{s}}(\mathscr{S}) \rightarrow \mathscr{H}^{\text {top }}$ est le foncteur dérivé total à droite du foncteur qui à $\mathscr{F}$ associe $\mathscr{F}(X))$;

(2) Pour tout $X \in \mathscr{S}$ et tout hyper-recouvrement $\mathscr{U} \rightarrow X$, la flèche

$$
\mathscr{F}(X) \rightarrow \operatorname{R}_{n \in \Delta} \mathscr{F}\left(\mathscr{U}_{n}\right)
$$

est un isomorphisme dans $\mathscr{H}^{\text {top }}$.

DÉfinition 5.2. - Soit $\mathscr{S}$ un site. On dira d'un objet de $\boldsymbol{\Delta}^{\mathrm{opp}} \operatorname{Prefais}(\mathscr{S})$ qu'il est acyclique s'il satisfait les conditions équivalentes du théorème précédent.

ThÉORÈme 5.3 ([11, theorem 1.3]). - Soit $X$ un espace topologique, soit $\mathscr{U}$ un hyper-recouvrement ouvert de $X$. Alors l'application

$$
\underset{n \in \Delta \text { opp }}{\operatorname{hocolim}} \operatorname{Sing} \mathscr{U}_{n} \rightarrow \operatorname{Sing} X
$$

est une équivalence faible d'ensembles simpliciaux.

Corollaire 5.4. - Soit $X$ un espace topologique, soit $K$ un ensemble simplicial fibrant. Alors, le préfaisceau simplicial sur $X$ qui à un ouvert $U$ associe le hom. interne d'ensembles simpliciaux hom $(\operatorname{Sing} U, K)$ est acyclique.

DÉFInITION 5.5. - Un espace topologique est localement contractile s'il possède une base d'ouverts formée d'ouverts contractiles.

Corollaire 5.6. - Soit $X$ un espace topologique localement contractile, $K$ un ensemble simplicial. On a un isomorphisme canonique

$$
\mathrm{R} \Gamma(X, K) \stackrel{\sim}{\rightarrow} \mathrm{R} \operatorname{hom}(\operatorname{Sing} X, K)
$$

où l'on a encore noté $K$ le (pré)faisceau simplicial constant associé à $K$. 
Tout d'abord, on peut supposer $K$ fibrant. Ensuite, on peut considérer l'application $K \rightarrow \operatorname{hom}(\operatorname{Sing} U, K)$ pour tout ouvert $U$ de $X$; cela définit un morphisme de préfaisceaux simpliciaux qui est une équivalence faible (locale) puisque $X$ est localement contractile (et que si $U$ est un ouvert contractile, alors l'ensemble simplicial $\operatorname{Sing} U$ est contractile). On peut utiliser le corollaire 5.4 pour conclure.

Remarque 5.7. - Ce corollaire 5.6 est une généralisation du théorème de comparaison entre la cohomologie singulière à coefficients entiers et la cohomologie du faisceau de groupes constant $\mathbf{Z}$ (appliquer le corollaire aux espaces d'Eilenberg-MacLane $K(\mathbf{Z}, n)$ pour $n \in \mathbf{N})$.

5.2. Le site des variétés à coins. - On note Coins la sous-catégorie pleine de la catégorie des espaces topologiques formée des variétés topologiques à coins, c'est-à-dire des espaces topologiques séparés et à base dénombrable admettant un recouvrement par des ouverts homéomorphes à des ouverts d'espaces topologiques de la forme $\mathbf{R}^{p} \times \mathbf{R}_{+}^{q}$ pour $(p, q) \in \mathbf{N}^{2(9)}$. Les espaces topologiques appartenant à Coins sont localement contractiles.

Il est clair que Coins est une catégorie essentiellement petite, on la munit de la topologie de Grothendieck induite par la topologie des espaces topologiques appartenant à Coins.

Proposition 5.8. - Soit $K$ un ensemble simplicial fibrant. Le préfaisceau sur Coins qui à une variété à coins $X$ associe hom(Sing $X, K)$ est acyclique.

Compte tenu du corollaire 5.4, ceci résulte du lemme suivant qui est lui-même une conséquence facile du théorème 5.1 :

Lemme 5.9. - Soit $\mathscr{F}$ un préfaisceau simplicial sur Coins. Pour tout $X \in$ Coins, on note $\mathscr{F} \mid X$ le préfaisceau simplicial sur l'espace topologique $X$ induit par $\mathscr{F}$. Les conditions suivantes sont équivalentes :

(1) $\mathscr{F}$ est acyclique;

(2) Pour tout $X \in$ Coins, $\mathscr{F}_{\mid X}$ est acyclique;

On note $a$ : Coins $\rightarrow \bullet$ le morphisme de sites évident. On obtient ainsi un foncteur faisceau constant $a^{\star}: \boldsymbol{\Delta}^{\mathrm{opp}}$ Ens $\rightarrow \boldsymbol{\Delta}^{\mathrm{opp}}$ Fais(Coins).

Muni de l'intervalle $[0,1]$, le site Coins devient un site avec intervalle; la proposition suivante nous sera très utile :

(9) On peut remarquer qu'il suffit de prendre $q \in\{0,1\}$ puisque $\mathbf{R}_{+}^{2}$ est homéomorphe à $\mathbf{R} \times \mathbf{R}_{+}$. Ainsi, je pourrais aussi bien parler de variétés topologiques à bord, mais je préfère parler de «coins » puisque cela rend plus fidèlement compte de la forme des simplexes.

TOME $135-2007-\mathrm{N}^{\mathrm{O}} 4$ 
Proposition 5.10. - Soit $K$ un ensemble simplicial. Le faisceau simplicial constant $a^{\star} K$ sur Coins est un $[0,1]$-local.

Cela se ramène à montrer que pour tout $X \in \mathbf{C o i n s , ~ l e ~ m o r p h i s m e ~ e ́ v i d e n t ~}$

$$
\mathrm{R} \Gamma(X, K) \rightarrow \mathrm{R} \Gamma(X \times[0,1], K)
$$

est un isomorphisme dans $\mathscr{H}^{\text {top }}$. Maintenant, comme $\operatorname{Sing}(X \times[0,1]) \rightarrow$ $\operatorname{Sing}(X)$ est une équivalence faible d'ensembles simpliciaux, la formule du corollaire 5.6 permet de conclure.

Remarque 5.11. - Il semble que les difficultés inhérentes à la démonstration de la proposition 5.10 aient été oubliées dans la démonstration de [24, proposition 3.3, page 120]. La démonstration qui suit (dans le cas particulier du groupe trivial) est plus complète.

THÉORÈme 5.12. - Le foncteur (pré)faisceau constant $a^{\star}: \boldsymbol{\Delta}^{\mathrm{opp}} \mathbf{E n s} \rightarrow$ $\operatorname{Esp}(\mathbf{C o i n s})$ induit une équivalence de catégories

$$
a^{\star}: \mathscr{H}^{\text {top }} \stackrel{\sim}{\rightarrow} \mathscr{H}(\text { Coins, }[0,1]) .
$$

On dispose d'un foncteur adjoint à droite $\mathrm{R}^{[0,1]} a_{\star}: \mathscr{H}($ Coins, $[0,1]) \rightarrow$ $\mathscr{H}^{\text {top }}$ à $a^{\star}$. Le fait que le morphisme de foncteurs issu de l'adjonction

$$
\operatorname{id}_{\mathscr{H}^{\text {top }}} \rightarrow \mathrm{R}^{[0,1]} a_{\star} a^{\star}
$$

soit un isomorphisme résulte du fait essentiel que l'image de $a^{\star}$ soit formée d'objets [0,1]-locaux (cf. proposition 5.10) : on vérifie que pour tout $K \in \boldsymbol{\Delta}^{\mathrm{opp}} \mathbf{E n s}$, on a des isomorphismes canoniques $\mathrm{R}^{[0,1]} a_{\star} a^{\star}=a_{\star} a^{\star} K=K$.

Il reste à montrer que l'autre morphisme d'adjonction

$$
a^{\star} \mathrm{R}^{[0,1]} a_{\star} \rightarrow \operatorname{id}_{\mathscr{H}(\text { Coins },[0,1])}
$$

est un isomorphisme, ce qui est l'objet du lemme suivant :

Lemme 5.13. - Soit $\mathscr{F}$ un préfaisceau simplicial sur Coins. On suppose que $\mathscr{F}$ est $[0,1]$-local. Alors $a^{\star} a_{\star} \mathscr{F} \rightarrow \mathscr{F}$ est une équivalence faible.

On a supposé $\mathscr{F}[0,1]$-local et on a vu que $a^{\star} a_{\star} \mathscr{F}$ était également [0,1]-local. Comme le morphisme évident $a_{\star} a^{\star} a_{\star} \mathscr{F} \rightarrow a_{\star} \mathscr{F}$ est un isomorphisme, il suffit d'appliquer le lemme suivant au morphisme $a^{\star} a_{\star} \mathscr{F} \rightarrow \mathscr{F}$ pour conclure :

Lemme 5.14. - Soit $\mathscr{F} \rightarrow \mathscr{G}$ un morphisme entre préfaisceaux simpliciaux sur Coins. On suppose que $\mathscr{F}$ et $\mathscr{G}$ sont $[0,1]$-locaux et que $a_{\star} \mathscr{F} \rightarrow a_{\star} \mathscr{G}$ est une équivalence faible, alors $\mathscr{F} \rightarrow \mathscr{G}$ est une équivalence faible. 
On peut supposer que $\mathscr{F}$ et $\mathscr{G}$ sont simplicialement fibrants. Étant donné que $\mathscr{F}$ et $\mathscr{G}$ sont $[0,1]$-locaux, pour tout objet $X \in$ Coins tel que le morphisme $X \rightarrow \bullet$ soit une $[0,1]$-équivalence faible, il vient que $\mathscr{F}(\bullet) \rightarrow \mathscr{F}(X)$ (resp. $\mathscr{G}(\bullet) \rightarrow \mathscr{G}(X))$ est une équivalence faible d'ensembles simpliciaux; comme $\mathscr{F}(\bullet) \rightarrow \mathscr{G}(\bullet)$ est une équivalence faible par hypothèse, il vient que $\mathscr{F}(X) \rightarrow$ $\mathscr{G}(X)$ est aussi une équivalence faible. Si $X \in$ Coins est un espace topologique contractile, l'application $X \rightarrow \bullet$ est évidemment une [0,1]-équivalence faible. Comme tous les objets de Coins sont localement contractiles, en passant aux foncteurs fibres, il vient que le morphisme $\mathscr{F} \rightarrow \mathscr{G}$ est une équivalence faible.

La construction du foncteur $a^{\star}$ s'étend trivialement aux $S^{1}$-spectres pour donner un foncteur $a^{\star}: \mathbf{S p t}^{S^{1}}(\bullet) \rightarrow \mathbf{S p t}^{S^{1}}$ (Coins, $\left.[0,1]\right)$.

THÉORÈme 5.15. - Le foncteur $a^{\star}$ induit des équivalences de catégories

$$
\begin{aligned}
& a^{\star}: S \mathcal{H}_{\mathrm{p}}^{S^{1}}(\bullet) \stackrel{\sim}{\rightarrow} \mathcal{S} \mathcal{H}_{\mathrm{p}}^{S^{1}}(\text { Coins, }[0,1]) ; \\
& a^{\star}: \mathcal{S} \mathcal{H}^{\text {top }} \stackrel{\sim}{\rightarrow} \mathcal{S} \mathcal{H}^{S^{1}}(\text { Coins },[0,1]) .
\end{aligned}
$$

Ce théorème se déduit assez trivialement du théorème 5.12.

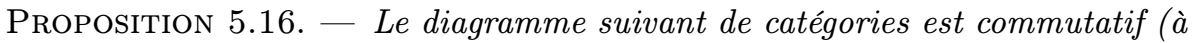
un isomorphisme canonique près) :

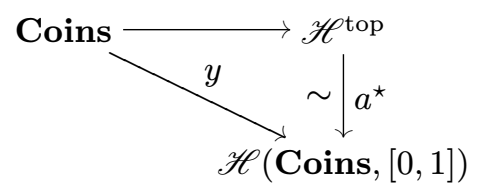

où le foncteur du haut est le foncteur évident et le foncteur $y$ : Coins $\rightarrow$ $\mathscr{H}$ (Coins, [0,1]) est induit par le plongement de Yoneda de Coins dans la catégorie des faisceaux sur Coins.

La proposition résulte du lemme suivant :

Lemme 5.17. - Pour tout objet $X \in$ Coins, il existe un zigzag canonique définissant un isomorphisme $y X \stackrel{\sim}{\rightarrow} a^{\star} \operatorname{Sing} X$ dans $\mathscr{H}$ (Coins, $\left.[0,1]\right)$.

Il convient de préciser le sens de ce lemme qui peut paraître subtil. La source $y X$ de cet isomorphisme est le faisceau d'ensembles sur Coins représenté par $X$, vu comme faisceau d'ensembles simpliciaux (discrets). Le but $a^{\star} \operatorname{Sing} X$ est le préfaisceau simplicial constant associé à l'ensemble simplicial Sing $X$. Simplicialement parlant, ces deux objets sont très différents, ce n'est qu'après $[0,1]$-localisation que l'on va pouvoir les identifier. 
La réalisation topologique de l'objet cosimplicial standard $\Delta^{\bullet}$ dans $\boldsymbol{\Delta}^{\mathrm{opp}}$ Ens définit un objet cosimplicial $\left|\Delta^{\bullet}\right|$ dans la catégorie Coins. On en déduit, comme dans [24, page 88] un foncteur

$$
\text { Sing }{ }^{\left|\Delta^{\bullet}\right|}: \Delta^{\mathrm{opp}} \text { Prefais }(\text { Coins }) \rightarrow \Delta^{\mathrm{opp}} \text { Prefais (Coins) }
$$

tel que si $\mathscr{X}$ est un préfaisceau simplicial sur Coins, alors $\operatorname{Sing}{ }^{\mid \Delta} \mid \mathscr{X}$ est la diagonale du préfaisceau bisimplicial

$$
(n, m) \longmapsto \operatorname{Hom}\left(\left|\Delta^{m}\right|, \mathscr{X}_{n}\right) .
$$

On dispose d'un morphisme fonctoriel évident

$$
\mathscr{X} \rightarrow \operatorname{Sing}^{\left|\Delta^{\bullet}\right|} \mathscr{X}
$$

qui est une $[0,1]$-équivalence faible (démonstration quasi-identique à celle de [24, corollary 3.8, page 89]). Considérons $\operatorname{Sing}^{\left|\Delta^{\bullet}\right|} y X$; c'est le préfaisceau simplicial sur Coins qui à un objet $U$ de Coins associe l'ensemble simplicial dont l'ensemble des $n$-simplexes est $\operatorname{Hom}_{\text {Coins }}\left(\left|\Delta^{n}\right| \times U, X\right)$, autrement dit $\operatorname{Sing}(\mathbf{C O}(U, X))$ où $\mathbf{C O}(U, X)$ est l'ensemble des applications continues $U \rightarrow X$ muni de la topologie compact-ouvert (noter que $U$ et $X$ sont séparés, et $U$ localement compact, cf. [12, §2, Chapter III]).

On a $a_{\star}\left(\operatorname{Sing}^{\mid \Delta^{\bullet}} \mid y X\right)=\operatorname{Sing} X$; pour montrer que le morphisme évident

$$
a^{\star} \operatorname{Sing} X \rightarrow \operatorname{Sing}^{\left|\Delta^{\bullet}\right|} y X
$$

est une équivalence faible de préfaisceaux simpliciaux sur Coins, il suffit de montrer que si $U \in$ Coins est contractile, alors le morphisme

$$
\operatorname{Sing} X \rightarrow \operatorname{Sing} \mathbf{C O}(U, X)
$$

est une équivalence faible d'ensembles simpliciaux, ce qui résulte du fait que

$$
X=\mathbf{C O}(\bullet, X) \rightarrow \mathbf{C O}(U, X)
$$

est une équivalence d'homotopie.

On a construit des $[0,1]$-équivalences faibles fonctorielles

$$
y X \rightarrow \operatorname{Sing}^{\mid \Delta}{ }^{\bullet} \mid X \leftarrow a^{\star} \operatorname{Sing} X,
$$

ce qui achève la démonstration de ce lemme.

\subsection{Topologie « étale » sur Coins}

DÉfinition 5.18. - Soit $X \in$ Coins. On note $X_{\text {ét la catégorie des flèches }}$ $Y \rightarrow X$ dans Coins qui sont «étales », c'est-à-dire des homéomorphismes locaux, autrement dit telles que pour tout $y \in Y$, il existe un ouvert $U$ de $Y$ contenant $y$ tel que l'application composée $U \rightarrow Y \rightarrow X$ soit un homéomorphisme de $U$ sur un ouvert de $X$. 
Comme les morphismes dans Coins sont les applications continues entre certains espaces topologiques et qu'il ne s'agit pas d'une notion différentielle, je préfère mettre le mot étale entre guillemets.

Il est aisé de montrer que la catégorie $X_{\text {ét }}$ admet des produits fibrés. On dispose de deux topologies de Grothendieck sur $X_{\text {ét }}$ :

- la topologie usuelle, associée à la prétopologie telle que si $Y \in X_{\text {ét }}, \operatorname{Cov}(Y)$ est formé des familles $\left(U_{i} \rightarrow Y\right)_{i \in I}$ de morphismes dans $X_{\text {ét }}$ telles que pour tout $i \in I, U_{i} \rightarrow Y$ soit un homéomorphisme de $U_{i}$ sur un ouvert de $Y$ et que l'application $\sqcup_{i \in I} U_{i} \rightarrow Y$ soit surjective;

- la topologie «étale », associée à la prétopologie telle que pour $Y \in X_{\text {ét }}$, $\operatorname{Cov}(Y)$ soit constitué des familles de morphismes $\left(U_{i} \rightarrow Y\right)_{i \in I}$ dans $X_{\text {ét }}$ (forcément «étales » au sens de la définition 5.18) telles que l'application $\sqcup_{i \in I} U_{i} \rightarrow Y$ soit surjective.

En mettant ensemble tous ces «petits sites », on obtient aussi deux topologies de Grothendieck sur Coins, la topologie usuelle de la sous-section 5.2 et la topologie «étale ». Ces deux topologies coïncident d'après le lemme suivant :

Lemme 5.19. - Soit $X \in$ Coins. La topologie usuelle et la topologie "étale » sur $X_{\text {ét }}$ coïncident.

Il est évident que la topologie étale est plus fine que la topologie usuelle. Il s'agit de montrer l'inclusion inverse. Soit donc $\left(Y_{i} \stackrel{p_{i}}{\longrightarrow} Y\right)_{i \in I}$ une famille de morphismes dans $X_{\text {ét }}$ appartenant à la prétopologie étale sur $X_{\text {ét }}$. Conformément à SGA 4 II 1.4, on va montrer que le crible de $Y$ qui lui est associé est couvrant pour la topologie usuelle. C'est très simple : pour tout point $y \in Y$, on peut choisir un indice $i_{y} \in I$ et un point $\tilde{y} \in Y_{i_{y}}$ tels que $p_{i}(\tilde{y})=y$. Choisissons maintenant un ouvert $V_{y}$ de $Y_{i_{y}}$ contenant $\tilde{y}$ et tel que l'application composée $V_{y} \rightarrow Y_{i_{y}} \rightarrow Y$ soit un homéomorphisme de $V_{y}$ sur un ouvert de $Y$. Le crible engendré par les morphismes $\left(Y_{i} \stackrel{p_{i}}{\longrightarrow} Y\right)_{i \in I}$ contient le crible engendré par les morphismes $\left(V_{y} \rightarrow Y\right)_{y \in Y}$, ce dernier crible est couvrant pour la topologie usuelle, ce qui permet de conclure.

Par conséquent, on notera $X_{\text {ét }}$ le site formé par la catégorie $X_{\text {ét }}$ munie de la topologie « étale » et on se souviendra que cette topologie coïncide avec la topologie usuelle.

5.4. L'application raisonnable $\mathrm{Sm} / \mathbf{C} \rightarrow$ Coins. - Soit $S$ un schéma noethérien muni d'un morphisme $\iota$ : Spec $\mathbf{C} \rightarrow S$. Pour tout objet $X$ de $\mathrm{Sm} / S$, on note $X(\mathbf{C})$ l'ensemble des $S$-morphismes $\operatorname{Spec} \mathbf{C} \rightarrow X$ muni de sa structure de variété C-différentielle (voir GAGA et SGA 1 XII 1.1). On définit ainsi un foncteur $\iota^{-1}: \mathrm{Sm} / S \rightarrow$ Coins par la formule $\iota^{-1}(X)=X(\mathbf{C})$. 
Proposition 5.20. - Le foncteur $\iota^{-1}: \mathrm{Sm} / S \rightarrow$ Coins définit une application continue raisonnable de sites $\iota:$ Coins $\rightarrow \mathrm{Sm} / S_{\mathrm{Nis}}$ (ou mieux Coins $\rightarrow$ $\left.\mathrm{Sm} / S_{\text {ét }}\right)$.

Commençons par le lemme suivant :

Lemme 5.21. - Soit $X \in \mathrm{Sm} / S$. Le foncteur $\iota^{-1}$ induit un foncteur $\iota_{X}^{-1}: X_{e ́ t} \rightarrow X(\mathbf{C})_{e ́ t}$ qui définit un morphisme de sites $\iota_{X}: X(\mathbf{C})_{e ́ t} \rightarrow X_{e ́ t}$ (cf. sous-section 5.3).

Il s'agit d'abord de montrer qu'un morphisme étale dans $\mathrm{Sm} / S$ est bien envoyé, par le foncteur $\iota^{-1}$, sur une application «étale» dans Coins : cela résulte du théorème d'inversion locale. On a bien un foncteur $\iota_{X}^{-1}: X_{\text {ét }} \rightarrow X(\mathbf{C})_{\text {ét }}$. Notons ensuite qu'un morphisme (étale) surjectif de schémas $f: X \rightarrow Y$ dans $\mathrm{Sm} / S$ induit une application surjective $X(\mathbf{C}) \rightarrow Y(\mathbf{C})$ : si $y \in Y(\mathbf{C})$, la fibre $X_{y}$ de $f$ au-dessus de $y$ est un schéma de type fini sur $\mathbf{C}$ et non vide, il possède un C-point ${ }^{(10)}$. Il en résulte que le foncteur $\iota_{X}^{-1}$ est continu; comme il commute aux limites projectives finies, on a bien un morphisme de sites (cf. SGA 4 IV 4.9.2).

On en déduit aussitôt le lemme suivant :

Lemme 5.22. - Soit $X \in \mathrm{Sm} /$ S. Si $\mathscr{F}$ est un préfaisceau simplicial acyclique sur $X(\mathbf{C})_{e ́ t}$, alors $\iota_{X, \star}(\mathscr{F})$ est un préfaisceau simplicial acyclique sur $X_{e ́ t}$.

On peut maintenant établir la proposition 5.20. Le lemme 5.21 a pour conséquence que le foncteur $\iota^{-1}: \mathrm{Sm} / S_{\text {ét }} \rightarrow$ Coins est continu. Montrer que ce foncteur définit une application continue raisonnable Coins $\rightarrow \mathrm{Sm} / S_{\text {ét }}$ (et donc aussi Coins $\rightarrow \mathrm{Sm} / S_{\mathrm{Nis}}$ ) revient à montrer que si un préfaisceau simplicial $\mathscr{F}$ sur Coins est acyclique alors $\iota_{\star}(\mathscr{F})$ est un préfaisceau simplicial acyclique sur $\mathrm{Sm} / S_{\text {ét }}$.

Soit donc $\mathscr{F}$ un préfaisceau simplicial acyclique sur Coins. On veut montrer que $\iota_{\star}(\mathscr{F})$ est acyclique sur $\mathrm{Sm} / S_{\text {ét }}$. D'après une variante du lemme 5.9 , il suffit

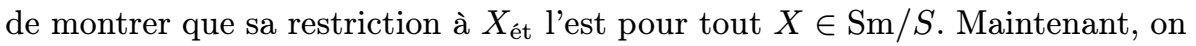
a un isomorphisme canonique

$$
\iota_{\star}(\mathscr{F})_{\mid X_{\text {ét }}}=\iota_{X, \star}\left(\mathscr{F}_{\mid X(\mathbf{C})_{\text {ét }}}\right)
$$

de préfaisceaux simpliciaux sur $X_{\text {ét }}$. Compte tenu du lemme 5.22, il suffit pour conclure de savoir que $\mathscr{F}_{\mid X(\mathbf{C})_{\text {ét }}}$ est acyclique, ce qui découle du lemme 5.9.

(10) Ceci serait faux si on remplaçait $\mathbf{C}$ par $\mathbf{R}$, il conviendrait alors d'utiliser la topologie de Nisnevich à la place de la topologie étale. 
Remarque 5.23. - Une difficulté de cette proposition provient du fait que la catégorie $\mathrm{Sm} / \mathbf{C}$ n'admette pas de produits fibrés, ce qui est un obstacle pour obtenir un morphisme de grands sites (un morphisme de sites donnant trivialement une application raisonnable de sites). On s'est donc ramené, grâce à un théorème de Dugger-Isaksen-Hollander (cf. théorème 5.1) à des considérations sur les petits sites (cf. lemme 5.9); cette approche est suggérée par [24, remark 1.21, page 102] qui envisage cette réduction pour l'obtention des applications raisonnables de sites $f_{\text {top }}: \mathrm{Sm} / T_{\text {top }} \rightarrow \mathrm{Sm} / S_{\text {top }}$ associées à un morphisme de schémas noethériens $f: T \rightarrow S$ et pour une topologie top parmi les topologies étale, de Nisnevich ou de Zariski. Pour obtenir la proposition 5.20, nous eussions également pu utiliser le théorème de Brown-Gersten comme dans [24, lemma 3.4, page 120] ou [24, proposition 1.20, page 103].

THÉORÈme 5.24. - Soit $S$ un schéma noethérien, soit $\iota: \operatorname{Spec} \mathbf{C} \rightarrow S$ un point complexe de $S$. Alors, $\iota$ induit des foncteurs :

$$
\iota^{\star}: \mathscr{H}(S) \rightarrow \mathscr{H}^{\text {top }} ; \quad \iota^{\star}: \mathscr{H}_{\bullet}(S) \rightarrow \mathscr{H}_{\bullet}^{\text {top }} .
$$

Ces foncteurs admettent des adjoints à droite.

Lemme 5.25. - Le foncteur $\iota^{-1}: \mathrm{Sm} / S \rightarrow$ Coins définit une application raisonnable de sites avec intervalles

$$
\iota:(\text { Coins, }[0,1]) \rightarrow\left(\mathrm{Sm} / S_{\mathrm{Nis}}, \mathbf{A}^{1}\right) .
$$

En vertu de [24, définition 3.16, page 92], comme on dispose déjà d'une application raisonnable de sites $\iota$ : Coins $\rightarrow \mathrm{Sm} / S_{\mathrm{Nis}}$ (cf. proposition 5.20), il suffit de montrer que pour tout $X \in \mathrm{Sm} / S$, le morphisme $\iota^{-1}\left(X \times \mathbf{A}^{1}\right) \rightarrow$ $\iota^{-1}(X)$ (autrement dit $X(\mathbf{C}) \times \mathbf{C} \rightarrow X(\mathbf{C})$ est une [0,1]-équivalence faible, ce qui est évident, $\mathbf{C}$ étant un espace topologique contractile).

Grâce à ce lemme, on dispose d'un foncteur $\mathscr{H}(S) \rightarrow \mathscr{H}$ (Coins, [0, 1]) (et de sa version pointée). D'après le théorème 5.12 , on en déduit un foncteur $\iota^{\star}: \mathscr{H}(S) \rightarrow \mathscr{H}^{\text {top }}$ (resp. $\mathscr{H}_{\bullet}(S) \rightarrow \mathscr{H}_{\bullet}^{\text {top }}$ ), ce qui achève la démonstration $\mathrm{du}$ théorème.

\subsection{Le foncteur triangulé $\mathcal{S H}(\mathbf{C}) \rightarrow \mathcal{S H} \mathcal{H}^{\text {top }}$}

ThÉORÈme 5.26. - Soit $S$ un schéma noethérien, soit $\iota: \operatorname{Spec} \mathbf{C} \rightarrow S$ un morphisme de schémas. Le morphisme $\iota$ donne naissance à un foncteur triangulé

$$
\iota^{\star}: \mathcal{S H}(S) \rightarrow \mathcal{S H} \mathcal{H}^{\text {top }}
$$

vérifiant $\iota^{\star}\left(X_{+}\right) \simeq X(\mathbf{C})_{+}$pour tout $X \in \mathrm{Sm} / S$. 
Dans un premier temps, observons que l'application raisonnable de sites avec intervalles construite au lemme 5.25 s'étend en une application raisonnable de sites suspendus avec intervalles (cf. définition 2.9) :

$$
\iota:\left(\text { Coins },[0,1], \mathbf{P}^{1}(\mathbf{C})\right) \rightarrow\left(\mathrm{Sm} / S_{\mathrm{Nis}}, \mathbf{A}^{1}, \mathbf{P}^{1}\right) .
$$

On en déduit un foncteur triangulé :

$$
\mathcal{S H}(S) \rightarrow \mathcal{S H}^{\mathbf{P}^{1}(\mathbf{C})}(\text { Coins, }[0,1]) .
$$

Compte tenu du corollaire 2.20, la proposition 5.16 (et plus précisément le lemme 5.17) permet de remplacer ci-dessus $\mathbf{P}^{1}(\mathbf{C})$ par le faisceau constant associé à l'ensemble simplicial Sing $\mathbf{P}^{1}(\mathbf{C})$. L'homéomorphisme classique entre la réalisation géométrique de l'ensemble simplicial $S^{2}$ et l'espace topologique $\mathbf{P}^{1}(\mathbf{C})$ détermine une équivalence faible $S^{2} \rightarrow \operatorname{Sing} \mathbf{P}^{1}(\mathbf{C})$. En appliquant une nouvelle fois le corollaire 2.20, on obtient finalement une équivalence de catégories entre $\mathcal{S H}^{\mathbf{P}^{1}(\mathbf{C})}(\mathbf{C o i n s},[0,1])$ et $\mathcal{S H}^{S^{2}}$ (Coins, [0,1]). D'après la proposition 2.22, cette dernière catégorie est équivalente à la catégorie $\mathcal{S} \mathcal{H}^{S^{1}}$ (Coins, $\left.[0,1]\right)$, qui à son tour est équivalente à $\mathcal{S} \mathcal{H}^{\text {top }}$ d'après le théorème 5.15. On a ainsi obtenu un foncteur $\iota^{\star}: \mathcal{S H}(S) \rightarrow \mathcal{S H} \mathcal{H}^{\text {top }}$ qui est triangulé d'après les résultats de la section 3 ; la dernière assertion du théorème résulte de la proposition 5.16.

\section{La construction naïve}

Dans cette section, on donne une version simplifiée $\mathcal{S H}_{\text {naïve }}^{T}(\mathscr{S}, I)$ de la catégorie homotopique stable $\mathcal{S H}^{T}(\mathscr{S}, I)$ d'un site suspendu avec intervalle $(\mathscr{S}, I, T)$. Cette catégorie présente l'avantage d'être définie très simplement à partir de la catégorie homotopique instable pointée $\mathscr{H}_{\bullet}(\mathscr{S}, I)$; nous allons supposer que $T$ est une suspension de sorte que la catégorie $\mathcal{S H}_{\text {naïve }}^{T}(\mathscr{S}, I)$ sera une catégorie additive, mais en général pas triangulée, contrairement à $\mathcal{S H}^{T}(\mathscr{S}, I)$ (cf. théorème 3.10). Ce qui empêchera le foncteur évident $\mathcal{S} \mathcal{H}^{T}(\mathscr{S}, I) \rightarrow \mathcal{S} \mathcal{H}_{\text {naïve }}^{T}(\mathscr{S}, I)$ d'être une équivalence de catégories sera la notion d'application stablement fantôme (cf. définition 6.6).

DÉFInition 6.1. - Soit $(\mathscr{S}, I, T)$ un site suspendu avec intervalle. On suppose qu'il existe un objet $T^{\prime} \in \mathscr{H}_{\bullet}(\mathscr{S}, I)$ tel que $T \simeq S^{1} \wedge T^{\prime}$. Un objet E de la catégorie $\mathcal{S H}_{\text {naïve }}^{T}(\mathscr{S}, I)$ est une suite $\left(\mathbf{E}_{n}\right)_{n \in \mathbf{N}}$ d'objets de $\mathscr{H}_{\bullet}(\mathscr{S}, I) \mathrm{mu}$ nis de morphismes d'assemblage $\sigma_{n}: T \wedge \mathbf{E}_{n} \rightarrow \mathbf{E}_{n+1}$ dans $\mathscr{H}_{\bullet}(\mathscr{S}, I)$ dont les morphismes adjoints $\mathbf{E}_{n} \rightarrow \mathrm{R} \mathbf{H o m} \bullet\left(T, \mathbf{E}_{n+1}\right)$ sont supposés être des isomorphismes. Un morphisme $\varphi: \mathbf{E} \rightarrow \mathbf{F}$ dans $\mathcal{S H}_{\text {naïve }}^{T}(\mathscr{S}, I)$ est simplement une 
suite de morphismes $\varphi_{n}: \mathbf{E}_{n} \rightarrow \mathbf{F}_{n}$ dans $\mathscr{H}_{\bullet}(\mathscr{S}, I)$ induisant des diagrammes commutatifs de la forme suivante dans $\mathscr{H}_{\bullet}(\mathscr{S}, I)$ :

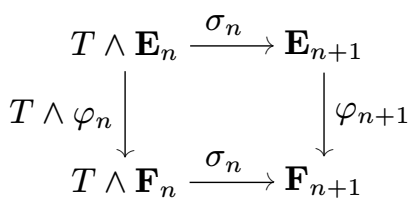

On dispose d'un foncteur oub: $\mathcal{S H}^{T}(\mathscr{S}, I) \rightarrow \mathcal{S H}_{\text {naïve }}^{T}(\mathscr{S}, I)$ : la catégorie $\mathcal{S} \mathcal{H}^{T}(\mathscr{S}, I)$ est équivalente à la sous-catégorie pleine $\mathcal{S H}_{\Omega}^{T}(\mathscr{S}, I)$ de $\mathcal{S H}_{\mathrm{p}}^{T}(\mathscr{S}, I)$ formée des $\Omega$-spectres, comme on dispose d'un foncteur évident $\mathcal{S H}_{\Omega}^{T}(\mathscr{S}, I) \rightarrow$ $\mathcal{S} \mathcal{H}_{\text {naïve }}^{T}(\mathscr{S}, I)$, on obtient le foncteur oub voulu.

Proposition 6.2. - La catégorie $\mathcal{S H}_{\text {naïve }}^{T}(\mathscr{S}, I)$ est une catégorie additive. De plus, le foncteur oub: $\mathcal{S H}^{T}(\mathscr{S}, I) \rightarrow \mathcal{S H}_{\text {naïve }}^{T}(\mathscr{S}, I)$ est additif.

On montre que $\mathcal{S H}_{\text {naïve }}^{T}(\mathscr{S}, I)$ est additive de façon très classique en utilisant la structure de cogroupe sur $S^{1}$ dans $\mathscr{H}_{\bullet}^{\text {top }}$, donnant naissance à une structure de cogroupe sur $T$ dans $\mathscr{H}_{\bullet}(\mathscr{S}, I)$ grâce à un isomorphisme $T \simeq S^{1} \wedge T^{\prime}$. Je préfère épargner ces détails au lecteur. Ensuite, le fait que le foncteur oub soit additif résulte simplement du fait qu'il commute aux produits (finis).

Proposition 6.3. - Le foncteur oub: $\mathcal{S H}^{T}(\mathscr{S}, I) \rightarrow \mathcal{S H}_{\text {naïve }}^{T}(\mathscr{S}, I)$ est conservatif $^{(11)}$, essentiellement surjectif et plein. De plus, si $\mathbf{E}$ est un objet de $\mathcal{S H}_{\text {naïve }}^{T}(\mathscr{S}, I)$, la catégorie des relèvements de $\mathbf{E}$ dans $\mathcal{S H}^{T}(\mathscr{S}, I)$ est équivalente à la catégorie ponctuelle (autrement dit, le relèvement est bien défini à isomorphisme unique près) si et seulement si

$$
\mathrm{R}^{1} \lim _{n \in \mathbf{N}} \operatorname{Hom}_{\mathscr{H}_{\bullet}(\mathscr{S}, I)}\left(S^{1} \wedge \mathbf{E}_{n}, \mathbf{E}_{n}\right)=0
$$

(les flèches de transition du système projectif étant les flèches évidentes).

Le fait que oub soit conservatif est évident. Il est aisé de montrer que ce foncteur est essentiellement surjectif : si on a un objet $\mathbf{E}$ de $\mathcal{S H}_{\text {naïve }}^{T}(\mathscr{S}, I)$, on peut représenter chaque objet $\mathbf{E}_{n}$ de $\mathscr{H}_{\bullet}(\mathscr{S}, I)$ par un faisceau simplicial pointé fibrant $\tilde{E}_{n}$ de sorte que le morphisme d'assemblage $\sigma_{n}: T \wedge \mathbf{E}_{n} \rightarrow \mathbf{E}_{n+1}$ soit la classe d'homotopie d'un authentique morphisme de faisceaux simpliciaux pointés $T \wedge \tilde{E}_{n} \rightarrow \tilde{E}_{n+1}$, ce qui définit bien un objet de $\mathcal{S H} \mathcal{H}^{T}(\mathscr{S}, I)$ dont l'image dans $\mathcal{S H}_{\text {naïve }}^{T}(\mathscr{S}, I)$ par le foncteur oub est isomorphe à $\mathbf{E}$.

(11) On rappelle qu'un foncteur $F: \mathscr{C} \rightarrow \mathscr{D}$ est dit conservatif si pour tout morphisme $f$ dans $\mathscr{C}$, le fait que $F(f)$ soit un isomorphisme implique que $f$ soit un isomorphisme.

TOME $135-2007-\mathrm{N}^{\mathrm{O}} 4$ 
LEMME 6.4. - Soit $\mathbf{E} \in \mathcal{S H}_{\mathrm{p}}^{T}(\mathscr{S}, I)$, soit $\mathbf{F} \in \mathcal{S H}_{\Omega}^{T}(\mathscr{S}, I)$. On a une suite exacte courte fonctorielle :

$$
\begin{aligned}
0 \rightarrow \mathrm{R}^{1} \lim _{k \in \mathbf{N}} \operatorname{Hom}_{\mathscr{H}_{\bullet}(\mathscr{S}, I)}\left(S^{1} \wedge \mathbf{E}_{k}, \mathbf{F}_{k}\right) & \rightarrow \operatorname{Hom}_{\mathcal{S} \mathcal{H}^{T}(\mathscr{S}, I)}(\mathbf{E}, \mathbf{F}) \\
& \rightarrow \lim _{k \in \mathbf{N}} \operatorname{Hom}_{\mathscr{H}}(\mathscr{S}, I)\left(\mathbf{E}_{k}, \mathbf{F}_{k}\right) \rightarrow 0
\end{aligned}
$$

Soit $\mathbf{E}$ un $T$-spectre. Pour $k \in \mathbf{N}$, on note $L_{k} \mathbf{E}$ le $T$-spectre

$$
\left(\mathbf{E}_{0}, \ldots, \mathbf{E}_{k}, T \wedge \mathbf{E}_{k}, T \wedge T \wedge \mathbf{E}_{k}, \ldots\right)
$$

dans lequel les premiers morphismes d'assemblage sont ceux de E, les autres étant les isomorphismes évidents. On obtient ainsi un système inductif de $T$ spectres

$$
L_{0} \mathbf{E} \rightarrow L_{1} \mathbf{E} \rightarrow L_{2} \mathbf{E} \rightarrow \ldots
$$

dont la colimite est E, c'est ce à quoi Jardine donne le nom de «layer filtration » dans [20]. Soit F un $\Omega$-spectre, la suite exacte de Milnor (voir [13, proposition 2.15, Chapter VI]) donne une suite exacte courte :

$$
\begin{aligned}
0 \rightarrow \mathrm{R}^{1} \lim _{k \in \mathbf{N}} \operatorname{Hom}_{\mathcal{S H} \mathcal{H}^{T}(\mathscr{S}, I)}\left(L_{k} \mathbf{E}[1], \mathbf{F}\right) & \rightarrow \operatorname{Hom}_{\mathcal{S H}(\mathscr{S}, I)}(\mathbf{E}, \mathbf{F}) \\
& \rightarrow \lim _{k \in \mathbf{N}} \operatorname{Hom}_{\mathcal{S} \mathcal{H}^{T}(\mathscr{S}, I)}\left(L_{k} \mathbf{E}, \mathbf{F}\right) \rightarrow 0
\end{aligned}
$$

On montre facilement que l'inclusion du $T$-spectre $F_{k} \mathbf{E}_{k}=\left(\bullet, \ldots, \bullet, \mathbf{E}_{k}, T \wedge\right.$ $\mathbf{E}_{k}, \ldots$ ) dans $L_{k} \mathbf{E}$ est une équivalence stable (cf. remarque 3.26), ainsi cette suite exacte courte prend la forme voulue.

Un cas particulier de ce lemme est le suivant :

LEMme 6.5. - Si $\mathbf{E}$ et $\mathbf{F}$ sont deux objets de $\mathcal{S H}_{\Omega}^{T}(\mathscr{S}, I)$, on a une suite exacte courte :

$$
\begin{aligned}
0 \rightarrow \mathrm{R}^{1} \lim _{k \in \mathbf{N}} \operatorname{Hom}_{\mathscr{H}_{\bullet}(\mathscr{S}, I)}\left(S^{1} \wedge \mathbf{E}_{k},\right. & \left.\mathbf{F}_{k}\right) \rightarrow \operatorname{Hom}_{\mathcal{S} \mathcal{H}^{T}(\mathscr{S}, I)}(\mathbf{E}, \mathbf{F}) \\
& \rightarrow \operatorname{Hom}_{\mathcal{S H}_{\text {naive }}^{T}(\mathscr{S}, I)}(\operatorname{oub} \mathbf{E}, \text { oub } \mathbf{F}) \rightarrow 0
\end{aligned}
$$

Revenons à la proposition 6.3. Il résulte du lemme précédent que le foncteur d'« oubli » oub: $\mathcal{S H}^{T}(\mathscr{S}, I) \rightarrow \mathcal{S H}_{\text {naïve }}^{T}(\mathscr{S}, I)$ est plein. Soit $\mathbf{E} \in \mathcal{S H}_{\text {naïve }}^{T}(\mathscr{S}, I)$. La catégorie des relèvements de $\mathbf{E}$ dans $\mathcal{S H}^{T}(\mathscr{S}, I)$ est la catégorie dont les objets sont les couples $(\tilde{\mathbf{E}}, \alpha)$ où $\tilde{\mathbf{E}} \in \mathcal{S H}^{T}(\mathscr{S}, I)$ et $\alpha$ est un isomorphisme oub $\tilde{\mathbf{E}} \stackrel{\alpha}{\rightarrow} \mathbf{E}$ dans $\mathcal{S H}_{\text {naïve }}^{T}(\mathscr{S}, I)$, un morphisme 
$\left(\tilde{\mathbf{E}}^{1}, \alpha_{1}\right) \rightarrow\left(\tilde{\mathbf{E}}^{2}, \alpha_{2}\right)$ étant un morphisme $f: \tilde{\mathbf{E}}^{1} \rightarrow \tilde{\mathbf{E}}^{2}$ dans $\mathcal{S} \mathcal{H}^{T}(\mathscr{S}, I)$ tel que le diagramme suivant soit commutatif dans $\mathcal{S} \mathcal{H}_{\text {naïve }}^{T}(\mathscr{S}, I)$ :

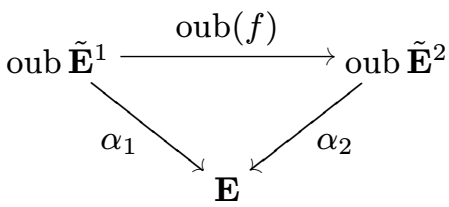

Comme oub est essentiellement surjectif, cette catégorie des relèvements est non vide ; dire qu'elle est équivalente à la catégorie ponctuelle revient à dire que si $\left(\tilde{\mathbf{E}}^{1}, \alpha_{1}\right)$ et $\left(\tilde{\mathbf{E}}^{2}, \alpha_{2}\right)$ sont deux relèvements de $\mathbf{E}$, il existe un unique morphisme entre ces deux objets dans la catégorie des relèvements. Le lemme précédent donne aussitôt le critère voulu, ce qui achève la démonstration de la proposition 6.3.

DÉFinition 6.6. - Si $\mathbf{E}$ et $\mathbf{F}$ sont deux objets de $\mathcal{S H}^{T}(\mathscr{S}, I)$, on note $\mathscr{F}(\mathbf{E}, \mathbf{F})$ le sous-groupe de $\operatorname{Hom}_{\mathcal{S} \mathcal{H}^{T}(\mathscr{S}, I)}(\mathbf{E}, \mathbf{F})$ formé des morphismes qui deviennent nuls après application du foncteur oub: $\mathcal{S H}^{T}(\mathscr{S}, I) \rightarrow \mathcal{S H}_{\text {naïve }}^{T}(\mathscr{S}, I)$. Les éléments de $\mathscr{F}(\mathbf{E}, \mathbf{F})$ sont appelés «applications stablement fantômes » (12).

La proposition suivante (qui est triviale) donne une caractérisation des applications stablement fantômes :

Proposition 6.7. - On se donne deux objets $\mathbf{E}$ et $\mathbf{F}$ de $\mathcal{S H}^{T}(\mathscr{S}, I)$. Soit $f: \mathbf{E} \rightarrow \mathbf{F}$ un morphisme dans $\mathcal{S H}^{T}(\mathscr{S}, I)$. Les conditions suivantes sont équivalentes :

- le morphisme $f$ est stablement fantôme, c'est-à-dire $f \in \mathscr{F}(\mathbf{E}, \mathbf{F})$;

- pour tout entier naturel $n$ et tout objet $X$ de $\mathscr{H}_{\bullet}(\mathscr{S}, I)$, l'application

$$
\operatorname{Hom}_{\mathcal{S} \mathcal{H}^{T}(\mathscr{S}, I)}\left(F_{n} X, \mathbf{E}\right) \stackrel{f \circ-}{\longrightarrow} \operatorname{Hom}_{\mathcal{S} \mathcal{H}^{T}(\mathscr{S}, I)}\left(F_{n} X, \mathbf{F}\right)
$$

induite par la composition par $f$ est nulle.

Corollaire 6.8. - Soit $n$ un entier naturel, soit $X$ un objet de $\mathscr{H}_{\bullet}(\mathscr{S}, I)$. Pour tout objet $\mathbf{F}$ de $\mathcal{S H}^{T}(\mathscr{S}, I)$, on a $\mathscr{F}\left(F_{n} X, \mathbf{F}\right)=0$.

Il est évident que l'on a défini un idéal bilatère $\mathscr{F}$ de la catégorie additive $\mathcal{S} \mathcal{H}^{T}(\mathscr{S}, I)$ au sens de [3]. La proposition 6.3 admet la conséquence suivante :

(12) Dans le cas classique, ces morphismes sont appelés « superphantom » dans [8].

TOME $135-2007-\mathrm{N}^{\circ} 4$ 
Proposition 6.9. - Le foncteur oub: $\mathcal{S H}^{T}(\mathscr{S}, I) \rightarrow \mathcal{S H}_{\text {naive }}^{T}(\mathscr{S}, I)$ induit une équivalence de catégories $\mathcal{S H}^{T}(\mathscr{S}, I) / \mathscr{F} \stackrel{\sim}{\rightarrow} \mathcal{S H}_{\text {naive }}^{T}(\mathscr{S}, I)$. Si $\mathbf{E}$ et $\mathbf{F}$ sont deux objets de $\mathcal{S H}^{T}(\mathscr{S}, I)$, on a une suite exacte courte :

$0 \rightarrow \mathscr{F}(\mathbf{E}, \mathbf{F}) \rightarrow \operatorname{Hom}_{\mathcal{S} \mathcal{H}^{T}(\mathscr{S}, I)}(\mathbf{E}, \mathbf{F}) \rightarrow \operatorname{Hom}_{\mathcal{S H}_{\text {naive }}^{T}(\mathscr{S}, I)}(\operatorname{oub} \mathbf{E}$, oub F $) \rightarrow 0$

Proposition 6.10. - L'idéal $\mathscr{F}$ de la catégorie additive $\mathcal{S H}^{T}(\mathscr{S}, I)$ est de carré nul. Autrement dit, si on se donne trois objets $\mathbf{E}, \mathbf{F}$ et $\mathbf{G}$ de $\mathcal{S} \mathcal{H}^{T}(\mathscr{S}, I)$ et des éléments $f \in \mathscr{F}(\mathbf{E}, \mathbf{F})$ et $g \in \mathscr{F}(\mathbf{F}, \mathbf{G})$, alors $g \circ f=0$.

Il s'agit de montrer que la flèche

$$
\mathscr{F}(\mathbf{E}, \mathbf{F}) \rightarrow \mathscr{F}(\mathbf{E}, \mathbf{G})
$$

induite par la composition à gauche par $g$ est nulle. On peut supposer que $\mathbf{E}$, $\mathbf{F}$ et $\mathbf{G}$ sont donnés par des $\Omega$-spectres. La fonctorialité évidente de la suite exacte du lemme 6.5 identifie la flèche ci-dessus à l'application

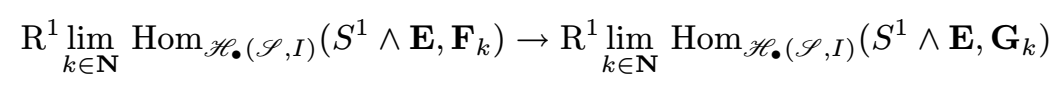

qui est induite par les morphismes $\mathbf{F}_{k} \rightarrow \mathbf{G}_{k}$ dans $\mathscr{H}_{\bullet}(\mathscr{S}, I)$ induits par $g$; comme $g \in \mathscr{F}(\mathbf{F}, \mathbf{G})$, les morphismes $\mathbf{F}_{k} \rightarrow \mathbf{G}_{k}$ sont nuls, ce qui permet de conclure.

Corollaire 6.11. - La catégorie additive $\mathcal{S H}_{\text {naive }}^{T}(\mathscr{S}, I)$ est pseudoabélienne.

Remarque 6.12. - Le bifoncteur $\mathscr{F}:\left(\mathcal{S H}^{T}(\mathscr{S}, I)\right)^{\mathrm{opp}} \times \mathcal{S H}^{T}(\mathscr{S}, I) \rightarrow \mathbf{A b}$ induit un foncteur $\mathscr{F}:\left(\mathcal{S H}_{\text {naïve }}^{T}(\mathscr{S}, I)\right)^{\mathrm{opp}} \times \mathcal{S H}_{\text {naïve }}^{T}(\mathscr{S}, I) \rightarrow$ Ab puisque l'idéal $\mathscr{F}$ est de carré nul. Cela permet de donner un sens à la notion d'application stablement fantôme entre objets de $\mathcal{S H}_{\text {naìve }}^{T}(\mathscr{S}, I)$, ce qui peut sembler assez paradoxal.

Remarque 6.13. - Les morphismes stablement fantômes sont quelque peu chimériques. On peut néanmoins montrer qu'il en existe : un exemple explicite en est donné en théorie homotopique des schémas (resp. en théorie de l'homotopie classique) dans [26, corollaire V.32] (resp. [26, remarque VI.16]). Dans le cas classique, un exemple plus compliqué apparaît dans [8, proposition 6.10]. 


\section{Appendice A}

\section{Catégories de modèles cellulaires}

La notion de catégorie de modèles cellulaire a été introduite dans [15, chapter XII]. Elle est utile dans la mesure où cette hypothèse de cellularité permet d'assurer l'existence de localisations à la Bousfield (cf. [15, theorem 4.1.1]). On se propose ici de donner une condition suffisante très facile à vérifier pour montrer qu'une catégorie de modèles est cellulaire.

DÉfinition A.1 (SGA 4 I 9.1). — Soit $\kappa$ un cardinal. Un ensemble ordonné filtrant $I$ est grand devant $\kappa$ si toute partie de $I$ de cardinal $\leq \kappa$ admet un majorant.

DÉfinition A.2 (SGA 4 I 9.3). — Soit $\mathcal{C}$ une catégorie admettant des limites inductives filtrantes. On dit d'un objet $X$ de $\mathcal{C}$ qu'il est accessible s'il existe un cardinal $\kappa$ tel que le foncteur $\operatorname{Hom}(X,-): \mathcal{C} \rightarrow$ Ens commute aux limites inductives filtrantes indexées par les ensembles ordonnés filtrants grands devant $\kappa$. Si $\kappa$ est un tel cardinal, on dit que $X$ est $\kappa$-accessible.

DÉfinition A.3. - Un monomorphisme effectif est un monomorphisme $A \rightarrow$ $B$ tel que la somme amalgamée $B \sqcup_{A} B$ existe et que $A$ soit l'égalisateur des deux morphismes évidents $B \rightarrow B \sqcup_{A} B$.

Proposition A.4. - Soit $\mathcal{C}$ une catégorie de modèles fermée à engendrement cofibrant. On suppose que tout objet de $\mathcal{C}$ est accessible et que les cofibrations sont des monomorphismes effectifs. Alors $\mathcal{C}$ est une catégorie de modèles fermée cellulaire.

La définition des catégories de modèles cellulaires (cf. [15, definition 12.1.1]) utilisant certains adjectifs dans un sens pouvant induire la confusion chez le lecteur, certains termes seront mis entre guillemets.

Choisissons des ensembles $I$ et $J$ de morphismes engendrant respectivement les cofibrations et les cofibrations triviales de $\mathcal{C}$. Les sources des morphismes de $J$ sont accessibles, ils sont en particulier «petits relativement à $I$ » au sens de [15, definition 10.5.12]. La condition (2) de [15, definition 12.1.1] est donc satisfaite. La condition (3) aussi, puisqu'elle exige que les cofibrations soient des monomorphismes effectifs. Il reste à montrer la condition (1) de la définition des catégories de modèles cellulaires : il faut montrer que les sources et buts des morphismes dans $I$ sont «compacts » au sens de [15, definition 11.4.1]. En fait, tous les objets de $\mathcal{C}$ le sont : 
Lemme A.5. - Soit $\mathcal{C}$ une catégorie admettant des limites inductives. Soit I une famille de morphismes dans $\mathcal{C}$. On suppose que tous les objets de $\mathcal{C}$ sont accessibles et que les complexes I-cellulaires relatifs (i.e. les composés transfinis d'images directes de sommes directes de morphismes appartenant à I) sont des monomorphismes. Alors, tout objet de $\mathcal{C}$ est «compact» au sens de $[15$, definition 11.4.1].

Soit $W$ un objet de $\mathcal{C}$. Il faut montrer qu'il existe un cardinal $\kappa$ tel que pour tout complexe $I$-cellulaire relatif $X \rightarrow Y^{(13)}$, toute flèche $W \rightarrow Y$ se factorise par $Y^{\prime} \rightarrow Y$ où $X \rightarrow Y^{\prime}$ est un sous-complexe $I$-cellulaire de $X \rightarrow Y$ de taille $\leq \kappa$ (autrement dit, on peut obtenir $Y^{\prime}$ en adjoignant à $X$ au plus $\kappa$ cellules de $X \rightarrow Y)$.

Choisissons un cardinal régulier $\kappa$ tel que $W$ et toutes les sources de flèches dans $I$ soient $\kappa$-accessibles. Notons $\mathscr{S}_{\kappa}$ l'ensemble ordonné des sous-complexes $I$-cellulaires $Y^{\prime}$ de $X \rightarrow Y$ de taille $\leq \kappa$. Cet ensemble ordonné $\mathscr{S}_{\kappa}$ est grand devant $\kappa$; l'objet $W$ étant $\kappa$-accessible, tout morphisme $W \rightarrow \operatorname{colim}_{Y^{\prime} \in \mathscr{S}_{\kappa}} Y^{\prime}$ se factorise par un $Y^{\prime} \in \mathscr{S}_{\kappa}$. Posons $Y_{\kappa}=\operatorname{colim}_{Y^{\prime} \in \mathscr{S}_{\kappa}}$. Pour conclure, il reste à montrer que le morphisme évident :

$$
Y_{\kappa} \rightarrow Y
$$

est un isomorphisme. Ce morphisme est un complexe $I$-cellulaire relatif. Pour montrer que c'est un isomorphisme, il suffit de vérifier que $Y_{\kappa}$ contient toutes les cellules de $X \rightarrow Y$. Si on tente d'adjoindre une cellule de $Y$ à $Y_{\kappa}$, on dispose d'un diagramme de la forme suivante, où le morphisme de gauche est dans $I$ :

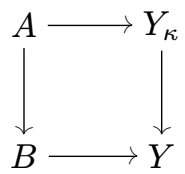

Comme $A$ est $\kappa$-accessible, la définition de $Y_{\kappa}$ fait que $A \rightarrow Y_{\kappa}$ se factorise par un $Y^{\prime} \in \mathscr{S}_{\kappa}$; par conséquent, le morphisme $B \rightarrow Y$ se factorise par $Y^{\prime \prime} \in \mathscr{S}_{\kappa}$ (et donc par $Y_{\kappa}$ ) où $Y^{\prime \prime}$ est le sous-complexe $I$-cellulaire obtenu en adjoignant la cellule considérée ici à $Y^{\prime}$. Ainsi, $Y_{\kappa} \rightarrow Y$ est un isomorphisme.

Remarque A.6. - On rappelle que dans une catégorie de faisceaux d'ensembles sur un site, tous les objets sont accessibles et les monomorphismes effectifs. Bien évidemment, ces propriétés sont encore vérifiées dans les catégories de faisceaux pointés ou de spectres. Le critère de la proposition A.4 s'applique donc aux catégories de modèles étudiées dans cet article.

(13) On suppose implicitement qu'une «présentation » de ce complexe a été choisie une fois pour toutes. Les sous-complexes de $X \rightarrow Y$ seront définis relativement à cette présentation. 


\section{BIBLIOGRAPHIE}

[1] Revêtements étales et groupe fondamental - Springer, 1971, Séminaire de Géométrie Algébrique du Bois Marie 1960-1961 (SGA 1), Dirigé par Alexandre Grothendieck. Augmenté de deux exposés de M. Raynaud, Lecture Notes in Mathematics, Vol. 224.

[2] Théorie des topos et cohomologie étale des schémas. Tome 1: Théorie des topos - Springer, 1972, Séminaire de Géométrie Algébrique du BoisMarie 1963-1964 (SGA 4), Dirigé par M. Artin, A. Grothendieck, et J. L. Verdier. Avec la collaboration de N. Bourbaki, P. Deligne et B. SaintDonat, Lecture Notes in Mathematics, Vol. 269.

[3] Y. André, B. Kahn \& P. O'Sullivan - « Nilpotence, radicaux et structures monoïdales », Rend. Sem. Mat. Univ. Padova 108 (2002), p. 107-291.

[4] J. Ayoub - «Les six opérations de Grothendieck et le formalisme des cycles évanescents dans le monde motivique », Thèse, Université Denis Diderot, Paris 7, 2006, http://www.institut.math.jussieu.fr/theses/ 2006/ayoub/these_ayoub.pdf.

[5] A. K. Bousfield \& D. M. KAN - Homotopy limits, completions and localizations, Springer, 1972, Lecture Notes in Mathematics, Vol. 304.

[6] K. S. Brown - « Abstract homotopy theory and generalized sheaf cohomology », Trans. Amer. Math. Soc. 186 (1974), p. 419-458.

[7] K. S. Brown \& S. M. Gersten - «Algebraic $K$-theory as generalized sheaf cohomology », in Algebraic K-theory, I : Higher K-theories (Proc. Conf., Battelle Memorial Inst., Seattle, Wash., 1972), Springer, 1973, p. 266-292. Lecture Notes in Math., Vol. 341.

[8] J. D. Christensen - «Ideals in triangulated categories : phantoms, ghosts and skeleta », Adv. Math. 136 (1998), p. 284-339.

[9] D.-C. CisinsKI - «Images directes cohomologiques dans les catégories de modèles », Ann. Math. Blaise Pascal 10 (2003), p. 195-244.

[10] D. Dugger, S. Hollander \& D. C. IsAKSEN - « Hypercovers and simplicial presheaves », Math. Proc. Cambridge Philos. Soc. 136 (2004), p. $9-51$.

[11] D. Dugger \& D. C. IsAKsen - «Topological hypercovers and $\mathbb{A}^{1}$ realizations », Math. Z. 246 (2004), p. 667-689.

[12] P. GABRIEL \& M. Zisman - Calculus of fractions and homotopy theory, Ergebnisse der Mathematik und ihrer Grenzgebiete, Band 35, Springer, 1967.

[13] P. G. Goerss \& J. F. JARdine - Simplicial homotopy theory, Progress in Mathematics, vol. 174, Birkhäuser Verlag, 1999. 
[14] A. Grothendieck \& J. Dieudonné - «Éléments de géométrie algébrique : IV. Étude locale des schémas et des morphismes de schémas (quatrième partie) », Publications mathématiques de l'IHES, vol. 4, 1960.

[15] P. S. HIRSCHHORN - Model categories and their localizations, Mathematical Surveys and Monographs, vol. 99, American Mathematical Society, 2003.

[16] M. Hovey - « Spectra and symmetric spectra in general model categories », J. Pure Appl. Algebra 165 (2001), p. 63-127.

[17] P. Hu - «S-modules in the category of schemes», Mem. Amer. Math. Soc. 161 (2003), p. 125.

[18] L. ILlusie - Complexe cotangent et déformations. I, Springer, 1971, Lecture Notes in Mathematics, Vol. 239.

[19] J. F. Jardine - «Simplicial presheaves », J. Pure Appl. Algebra 47 (1987), p. $35-87$.

[20] _ « Motivic symmetric spectra », Doc. Math. 5 (2000), p. 445-553.

[21] K. Kato \& S. SAITO - «Global class field theory of arithmetic schemes », in Applications of algebraic $K$-theory to algebraic geometry and number theory, Part I, II (Boulder, Colo., 1983), Contemp. Math., vol. 55, Amer. Math. Soc., 1986, p. 255-331.

[22] G. Maltsiniotis - «Structure triangulée sur les catégories des coefficients d'un dérivateur triangulé », en préparation.

[23] _ _ «a $K$-théorie d'un dérivateur triangulé », Contemp. Math. 431 (2007), p. 341-368, (suivi d'un appendice par B. Keller).

[24] F. Morel \& V. Voevodsky - « $\mathbf{A}^{1}$-homotopy theory of schemes », Publ. Math. I.H.E.S. (1999), p. 45-143 (2001).

[25] D. G. QuilLen - Homotopical algebra, Lecture Notes in Mathematics, No. 43, Springer, 1967.

[26] J. Riou - «Opérations sur la $K$-théorie algébrique et régulateurs via la théorie homotopique des schémas », Thèse, Université Paris 7 - Denis Diderot, 2006, http://www.institut.math. jussieu.fr/theses/2006/riou/ these-riou.pdf.

[27] J-P. SERRE - « Géométrie algébrique et géométrie analytique», Ann. Inst. Fourier, Grenoble 6 (1955-1956), p. 1-42.

[28] R. W. Thomason - «Algebraic $K$-theory and étale cohomology », Ann. Sci. École Norm. Sup. (4) 18 (1985), p. 437-552.

[29] J.-L. Verdier - «Des catégories dérivées des catégories abéliennes », Astérisque (1996), p. 253 pp. (1997).

[30] V. Voevodsky - « $\mathbf{A}^{1}$-homotopy theory », in Proceedings of the International Congress of Mathematicians, Vol. I (Berlin, 1998), 1998, p. 579-604. 